\title{
A-train CALIOP and MLS observations of early winter Antarctic polar stratospheric clouds and nitric acid in 2008
}

\author{
A. Lambert ${ }^{1}$, M. L. Santee ${ }^{1}$, D. L. Wu ${ }^{1, *}$, and J. H. Chae ${ }^{1, * *}$ \\ ${ }^{1}$ NASA Jet Propulsion Laboratory, California Institute of Technology, Pasadena, California, USA \\ *now at: NASA Goddard Space Flight Center, Greenbelt, Maryland, USA \\ ** now at: Department of Geological Sciences, University of Texas at Austin, Austin, Texas, USA
}

Correspondence to: A. Lambert (alyn.lambert@jpl.nasa.gov)

Received: 4 October 2011 - Published in Atmos. Chem. Phys. Discuss.: 31 October 2011

Revised: 9 March 2012 - Accepted: 12 March 2012 - Published: 23 March 2012

\begin{abstract}
A-train Cloud-Aerosol Lidar with Orthogonal Polarization (CALIOP) and Microwave Limb Sounder (MLS) observations are used to investigate the development of polar stratospheric clouds (PSCs) and the gas-phase nitric acid distribution in the early 2008 Antarctic winter. Observational evidence of gravity-wave activity is provided by Atmospheric Infrared Sounder (AIRS) radiances and infrared spectroscopic detection of nitric acid trihydrate (NAT) in PSCs is obtained from the Michelson Interferometer for Passive Atmospheric Sounding (MIPAS). Goddard Earth Observing System Data Assimilation System (GEOS-5 DAS) analyses are used to derive Lagrangian trajectories and to determine temperature-time histories of air parcels. We use CALIOP backscatter and depolarization measurements to classify PSCs and the MLS measurements to determine the corresponding gas-phase $\mathrm{HNO}_{3}$ as a function of temperature. For liquid PSCs the uptake of $\mathrm{HNO}_{3}$ follows the theoretical equilibrium curve for supercooled ternary solutions (STS), but at temperatures about $1 \mathrm{~K}$ lower as determined from GEOS-5. In the presence of solid phase PSCs, above the ice frost-point, the $\mathrm{HNO}_{3}$ depletion occurs over a wider range of temperatures $(+2$ to $-7 \mathrm{~K})$ distributed about the NAT equilibrium curve. Rapid gas-phase $\mathrm{HNO}_{3}$ depletion is first seen by MLS from from 23-25 May 2008, consisting of a decrease in the volume mixing ratio from $14 \mathrm{ppbv}$ (parts per billion by volume) to $7 \mathrm{ppbv}$ on the $46-32 \mathrm{hPa}$ (hectopascal) pressure levels and accompanied by a $2-3$ ppbv increase by renitrification at the $68 \mathrm{hPa}$ pressure level. The observed region of depleted $\mathrm{HNO}_{3}$ is substantially smaller than the region bounded by the NAT existence temperature threshold. Temperature-time histories of air parcels demonstrate that
\end{abstract}

the depletion is more clearly correlated with prior exposure to temperatures a few kelvin above the frost-point. From the combined data we infer the presence of large-size NAT particles with effective radii $>5-7 \mu \mathrm{m}$ and low NAT number densities $<1 \times 10^{-3} \mathrm{~cm}^{-3}$. This denitrification event is observed close to the pole in the Antarctic vortex before synoptic temperatures first fall below the ice frost point and before the widespread occurrence of large-scale NAT PSCs. An episode of mountain wave activity detected by AIRS on 28 May 2008 led to wave-ice formation in the rapid cooling phases over the Antarctic Peninsula and Ellsworth Mountains, seeding an outbreak of NAT PSCs that were detected by CALIOP and MIPAS. The NAT clouds formed at altitudes of $18-26 \mathrm{~km}$ in a polar freezing belt and appear to be composed of relatively small particles with estimated effective radii of around $1 \mu \mathrm{m}$ and high NAT number densities $>0.2 \mathrm{~cm}^{-3}$. This NAT outbreak is similar to an event previously reported from MIPAS observations in mid-June 2003.

\section{Introduction}

Polar stratospheric clouds (PSCs) are the key mediator in polar ozone depletion by enabling heterogeneous processes that release halogens from relatively stable reservoir species. The liberated halogens are dissociated by ultraviolet photolysis on the return of sunlight in the polar spring and then proceed to destroy stratospheric ozone in catalytic photochemical cycles (e.g., Solomon, 1999). Sequestration of nitric acid into PSCs from the gas phase alters the balance of partitioning of active nitrogen species that transfer reactive halogens back to 
their inert reservoir form. Another process that accentuates ozone destruction through removal of this reconversion pathway is the gravitational sedimentation of large PSC particulates containing nitric acid hydrates, which can physically remove nitrogen oxides and water vapor from a large extent of the polar lower stratosphere.

Many different measurement techniques have been used in the quest for understanding the formation of PSCs. Balloon and aircraft measurements using in-situ optical particle counters, backscatter sondes, chemiluminescence and mass spectrometry have played an important role in determining particle size distributions and compositions (Lowe and MacKenzie, 2008). The ability of infrared remote sounding to discriminate sulfate aerosols from PSCs was first demonstrated by Kinne et al. (1989) using the JPL MkIV interferometer on board the DC-8 aircraft. Lidar measurements of backscatter and depolarization from ground-based, airborne and satellite platforms provide information for PSC type classification (Toon et al., 1990; Adriani et al., 2004; Pitts et al., 2009). The PSC phase can be determined from depolarization (Biele et al., 2001), and particle size can be inferred from color ratios based on the wavelength dependent backscatter at two or more wavelengths (Toon et al., 2000). However, lidar measurements do not determine the stoichiometric composition of PSCs, and the classification schemes are based on optical models. Multi-wavelength detection allows the estimation of surface area, volume, effective particle size and composition. Discrimination between solid and liquid PSCs is possible using multi-wavelength extinction measurements (Larsen et al., 2004, and references therein).

Solar occultation measurements from satellites have been used for over $30 \mathrm{yr}$ to investigate PSCs, evolving from the single channel 1- $\mu \mathrm{m}$ Stratospheric Aerosol Measurement (SAM-II) instrument and continuing with a series of multi-channel instruments, the Stratospheric Aerosol and Gas Experiment (SAGE I, II, III), the Improved Limb Atmospheric Spectrometer (ILAS I, II) and the Polar Ozone and Aerosol Measurement (POAM II, III). These observations have been important in establishing a long-term PSC climatology (Fromm et al., 2003), but their potential is limited by the inherently low sampling frequency of the solar occultation technique.

Space-based attempts to determine the composition of PSCs from their spectral infrared signatures were made using instruments on the Upper Atmosphere Research Satellite (UARS) (Taylor et al., 1994; Massie et al., 1997; Hervig, 1999), which also provided contemporaneous observations of temperature and gas species. These early investigations of PSC composition from UARS were subsequently improved upon by the Cryogenic Spectrometers and Telescopes for the Atmosphere (CRISTA) and the Michelson Interferometer for Passive Atmospheric Sounding (MIPAS) instruments (Spang and Remedios, 2003; Höpfner et al., 2006b).

Complementary measurements obtained from the CloudAerosol Lidar with Orthogonal Polarization (CALIOP) on the Cloud-Aerosol Lidar Infrared Pathfinder Satellite Observations (CALIPSO) satellite and the Aura Microwave Limb Sounder (MLS) provide a rich dataset for the study of PSCs and their effects on the vertical redistribution of nitric acid and water vapor. In the following sections we show that the near-simultaneous measurements (within $30 \mathrm{~s}$ and $10 \mathrm{~km}$ ) by CALIOP and MLS from the A-train can provide important information on the characteristics of PSCs and the associated atmospheric composition. Microwave gas-phase measurements are insensitive to the presence of PSCs along the lineof-sight, unlike solar occultation and infrared measurements, e.g., MIPAS (Wang et al., 2007), which must attempt to separate the effects of aerosol and cloud contamination on the gas-phase measurements. Observations of PSCs by CALIOP have been reported by Noel et al. $(2008,2009)$ and Pitts et al. (2007, 2009, 2011). Comparisons of PSC measurements by CALIOP and MIPAS of cloud top height and type classifications have been presented by Höpfner et al. (2009). Previous investigations using the combined CALIOP and MLS data (Pitts et al., 2007, 2009, 2011) have involved vortex-averaged analyses of PSCs and dynamics/chemistry. The analyses presented here are based on measurements during the early 2008 Antarctic winter and involve: (i) comparison of the modeled and observed gas-phase uptake of $\mathrm{HNO}_{3}$ in liquid and solid particle PSCs, (ii) the onset of denitrification in the polar vortex, and (iii) observations of the development of solid particle PSCs in a polar freezing belt (Tabazadeh et al., 2001; Höpfner et al., 2006a).

In this paper, we first review in Sect. 2 properties and observations of PSCs relevant to the interpretation and discussion of our studies. Section 3 describes the satellite instruments, measurements and data analysis methods. Results are presented in Sect. 4, and finally conclusions are drawn in Sect. 5.

\section{Key aspects of PSC formation and evolution}

\subsection{Composition}

Polar stratospheric cloud particles have been determined to be composed of three main types (Peter, 1997; Lowe and MacKenzie, 2008), consisting of the three main species $\mathrm{H}_{2} \mathrm{SO}_{4}, \mathrm{HNO}_{3}$ and $\mathrm{H}_{2} \mathrm{O}$, with trace contributions from $\mathrm{HCl}$, $\mathrm{HOCl}$ and $\mathrm{HBr}$. Type Ia are solid non-spherical particles composed of nitric acid hydrates, most likely nitric acid trihydrate (NAT) (Hanson and Mauersberger, 1988) or possibly nitric acid dihydrate (NAD) (Worsnop et al., 1993). Only NAT particles have been detected as a solid component by in situ composition measurements (Voigt et al., 2000a). Type Ia particle sizes are typically less than a few microns in size, but under certain conditions they can grow to much larger sizes (Fahey et al., 2001), the efficient sedimentation of which leads consequently to permanent removal of reactive nitrogen (denitrification) from the polar lower stratosphere. 
Type Ib PSCs are sub-micron size spherical liquid droplets and are composed of supercooled ternary solutions (STS) of $\mathrm{H}_{2} \mathrm{SO}_{4}, \mathrm{HNO}_{3}$ and $\mathrm{H}_{2} \mathrm{O}$ (Carlsaw et al., 1994). Properties of the two distinct Type I PSC classes were derived from lidar depolarization characteristics (Browell et al., 1990; Toon et al., 1990; Stefanutti et al., 1995) and from in situ measurements of aerosol volume/temperature relationships (Dye et al., 1992, 1996). Type II PSCs are water-ice particles that form only under the coldest conditions below the ice frost point, and so are less frequently created in the Arctic than in the Antarctic. Ice particles can grow to very large sizes that sediment rapidly and lead to permanent removal of water (dehydration) from the polar lower stratosphere.

\subsection{Thermodynamics}

Thermodynamic calculations are used to determine threshold existence temperatures of the PSC types, which are dependent on the ambient partial pressures of $\mathrm{H}_{2} \mathrm{O}$ in the case of the ice frost point, $T_{\text {ICE }}$ (Murphy and Koop, 2005), and also $\mathrm{HNO}_{3}$ for $T_{\mathrm{NAT}}$ (Hanson and Mauersberger, 1988) and $T_{\text {NAD }}$ (Worsnop et al., 1993). Under typical conditions in the polar stratosphere, NAT is thermodynamically stable at temperatures below $T_{\mathrm{NAT}} \sim T_{\mathrm{ICE}}+7 \mathrm{~K}$. In the case of the liquid STS there is no particular threshold temperature and the STS drops, forming on the ubiquitous background sulfate aerosol, grow slowly by condensation. As the temperature of a sulfate aerosol droplet decreases, there is an uptake of $\mathrm{H}_{2} \mathrm{SO}_{4}$ and $\mathrm{H}_{2} \mathrm{O}$ from the gas phase. As the temperature decreases further the aerosol remains a supercooled liquid, and a further uptake of $\mathrm{HNO}_{3}$ from the gas into the condensed phase occurs a few kelvin below the NAT point close to $T_{\mathrm{STS}} \sim T_{\mathrm{NAT}}-$ 3.5 K (Carslaw et al., 1997; Drdla et al., 2003). Other gases, including halogen reservoir species, dissolve into the STS drops. The rate of sedimentation of STS cannot lead to rapid denitrification (Lowe and MacKenzie, 2008) as the drops are limited in size to below $1 \mu \mathrm{m}$.

\subsection{Nucleation processes}

The mechanisms of solid particle nucleation or conversion of STS into NAT or ice are unclear (Lowe and MacKenzie, 2008). Laboratory measurements have determined that homogeneous nucleation of ice from STS occurs at temperatures a few kelvin below the ice frost point (Koop et al., 1998), but homogeneous nucleation of NAT from STS occurs at too low a rate (Koop et al., 1997b) to account for observations of PSC formation. Although ice particles can act as sites for heterogeneous NAT nucleation, some other nucleation process must be at work to account for observations of NAT in air parcels known not to have experienced temperatures below the ice frost point (Drdla et al., 2002; Larsen et al., 2004; Pagan et al., 2004; Voigt et al., 2005). Larsen et al. (1997) presented evidence from Arctic balloonborne backscatter sonde observations for the effects of tem- perature history on NAT PSC formation, such as temperature oscillations and duration of exposure below $T_{\mathrm{NAT}}$. In a study of Antarctic POAM PSC observations, Steele et al. (2002) concluded that other factors besides temperature history are likely to be a critical aspect of NAT PSC formation. The presence of aerosol impurities such as soot, meteoritic debris, and effects of ionization by cosmic rays have been proposed as agents which may act to promote freezing (Zondlo et al., 2000; Yu, 2004; Voigt et al., 2005). However, studies of the bulk freezing of STS containing meteoritic particles and other nuclei (Biermann et al., 1996), and of the nucleation rate of NAT on clay mineral particles (Hatch et al., 2008), have indicated rates much too slow to be significant, even for large supersaturations in the typical polar stratosphere.

\subsection{Role of meteorology}

\subsubsection{Synoptic-scale dynamics}

Every winter the establishment of a stable Antarctic polar vortex provides a setting for the development of very low synoptic-scale temperatures below the threshold for PSC existence, $T_{\mathrm{NAT}}$, for several months. In contrast, the Arctic features stronger planetary wave activity that often disrupts the weaker vortex, and a tendency for stratospheric warmings to develop limits the occurrence and maintenance of temperatures low enough to initiate and sustain PSC formation. Teitelbaum et al. (2001) analyzed the effects of tropospheric meteorological forcing on PSC and ozone minihole formation and concluded that synoptic-scale dynamics is the dominant mechanism in the Arctic and, in an 18-yr record of solar occultation measurements, Fromm et al. (2003) found a strong association of PSC formation with elevated mean tropopause height. CALIPSO and CloudSat observations have been used to determine that a large fraction of Antarctic PSCs are probably linked to underlying synoptic-scale deep tropospheric cloud systems (Wang et al., 2008; Adhikari et al., 2010).

\subsubsection{Mesoscale temperature fluctuations}

The Arctic has been the choice of most of the research on mountain wave NAT formation (e.g., Carslaw et al., 1998) in preference to the same process in the Antarctic, where the synoptic temperatures are frequently lower than the ice frost point. In both polar regions, mesoscale temperature fluctuations induced by orographically forced gravity waves are an important mechanism for the production of PSCs (Carslaw et al., 1998; McDonald et al., 2009; Alexander et al., 2011). These short time-scale temperature fluctuations subject particles to rapid cooling/heating rates that can force phase transitions (Koop et al., 1997a) and also cause particles to be driven away from an equilibrium state as the liquid and solid phases react to changes in ambient conditions (Lowe and MacKenzie, 2008). The time constant for gas-phase diffusion of $\mathrm{HNO}_{3}$ can be $\sim$ hours, whereas $\mathrm{H}_{2} \mathrm{O}$ reaches equilibrium 
in seconds (Meilinger et al., 1995; Lowe and MacKenzie, 2008). This can result in a hysteresis effect in the STS $\mathrm{HNO}_{3}$ mass fraction. Observations have frequently shown significant non-equilibrium effects in PSC development (Peter et al., 1994; Voigt et al., 2000b; Lowe et al., 2006). Biele et al. (2001) presented lidar observations of Type Ia and Ib PSCs that showed optical properties with significant deviations from those expected for particles in thermodynamic equilibrium. PSCs classified as Type Ib liquid were often found to display a significant perpendicular backscatter component, indicating the presence of mixed phase clouds with a small fraction of solid particles amongst a majority of liquid particles. Type Ia solid PSCs were shown to have generally much lower backscatter than expected for particles in NAT equilibrium.

\subsection{Observations of NAT PSCs in a polar freezing belt}

Recent work has highlighted the role of mountain wave events over the Antarctic Peninsula in triggering heterogeneous nucleation of NAT on ice and explaining the formation of a circumpolar belt of NAT PSCs observed by satellite measurements. Localized cooling events by mountain waves have been shown to lead to production of NAT particles (Höpfner et al., 2006a; Eckermann et al., 2009), which can persist and grow downstream after nucleation through an ice-seeding mechanism (Fueglistaler et al., 2002a; Dhaniyala et al., 2002). Höpfner et al. (2006a) used the MIPAS measurements in the Antarctic from May-June 2003 to investigate the initial stages of NAT PSC formation. They observed a sudden onset of NAT PSCs over a few days from 1012 June 2003 in a region downstream of the Antarctic Peninsula within a polar freezing belt (Tabazadeh et al., 2001), but they noticed the absence of PSCs within the belt in the region upstream of the Peninsula despite similarly low synoptic temperatures. Through microphysical modeling and mountain wave hindcasts, Höpfner et al. (2006a) determined that the likely cause was the heterogeneous nucleation of NAT on ice particles that had formed over the Palmer Peninsula and noted that previous observations of PSCs were also associated with formation near this location and subsequent further development downstream (Cariolle et al., 1989; Ricaud et al., 1995). Subsequently, Atmospheric Infrared Sounder (AIRS) data were used by Eckermann et al. (2009) to show that the MIPAS PSC episode followed the occurrence of $\pm 10 \mathrm{~K}$ amplitude temperature perturbations, which provided the mesoscale source of rapid cooling needed to explain the ice formation.

\subsection{Denitrification in the polar stratosphere}

Denitrification has been observed without accompanying significant dehydration in both polar regions, and it is therefore well established that irreversible removal of $\mathrm{HNO}_{3}$ from the stratosphere does not require large scale processes operating below the ice frost point (Fahey et al., 1990; Santee et al., 2000; Tabazadeh et al., 2000; Drdla et al., 2003; Voigt et al., 2005).

\subsubsection{Large particle sedimentation}

Very large (5-10- $\mu \mathrm{m}$ radius) particles were detected above the ice frost point by instruments flown on the ER-2 in 1989 (Dye et al., 1992). Subsequently, during the combined SAGE III Ozone Loss and Validation Experiment (SOLVE) and the Third European Stratospheric Experiment on Ozone (THESEO 2000) campaign, small concentrations of large nitric acid-containing particles ("NAT-rocks") were observed (Fahey et al., 2001; Fueglistaler et al., 2002b), and these measurements have been prime motivators for theoretical and modeling investigations. Wofsy et al. (1990) proposed a mechanism for denitrification without significant dehydration by predicting that Type II PSC particles would sediment through Type I PSC regions below and be protected from evaporation by accreting a NAT coating from the gas phase during their passage through NAT-supersaturated air. Laboratory experiments by Aguzzi and Rossi (2001) support this accretion mechanism, whereas Biermann et al. (1998) reported that it is not efficient, and proposed an alternative mechanism whereby NAT forms from the liquid phase surrounding the ice core.

Carslaw et al. (2002) modeled the growth, evaporation, sedimentation, and advection of NAT (and NAD) particles in the January-March 2000 Arctic vortex, but limitations caused by the lack of contemporaneous information on the $\mathrm{HNO}_{3}$ field above the aircraft prevented the possibility to distinguish between NAT and NAD compositions for the particles. However, in a similar study McKinney et al. (2004) used Airborne Submillimeter Radiometer (ASUR) inner and outer vortex averaged $\mathrm{HNO}_{3}$ gas phase profiles to estimate denitrified and unperturbed $\mathrm{HNO}_{3}$ profiles and showed that modeled development of NAD particles resulted in maximum sizes smaller than those actually observed, whereas NAT particles produced a better size comparison. Von König et al. (2002) combined ASUR gas-phase $\mathrm{HNO}_{3}$ measurements with lidar to determine a likely NAT composition for particles in the Arctic; however, this investigation was limited by the low vertical resolution of the ASUR instrument (6-10 km).

\subsubsection{NAT condensation/renitrification cycle}

In the absence of Type II PSCs, only solid particle NAT (NAD) PSCs with low number densities, possibly arising from preferential nucleation, and their subsequent more rapid growth can lead to extensive denitrification and permanent removal of $\mathrm{HNO}_{3}$ from the stratosphere (Jensen et al., 2002). Efficient irreversible denitrification requires the formation and growth of particles containing $\mathrm{HNO}_{3}$ of sufficient size to gravitationally sediment in a matter of days rather than over 
many weeks. The particles responsible must be solid since liquid particles cannot grow by condensation to sufficient size to undergo rapid sedimentation (Lowe and MacKenzie, 2008). Jensen et al. (2002) simulated the effects of PSC composition, number density and lifetime on denitrification. Their findings indicate that NAT number densities in the range $3 \times 10^{-3}$ to $10^{-2} \mathrm{~cm}^{-3}$ provide the most efficient route to denitrification, with $50 \%$ depletion of the gasphase $\mathrm{HNO}_{3}$ occurring at around $20 \mathrm{~km}$ for a cloud lifetime of about one week. Nucleated NAT particles evolved into a distribution with an effective radius larger than $5 \mu \mathrm{m}$ over a four day period as they gravitationally sedimented. Following a cooling/warming cycle, the residual gas-phase $\mathrm{HNO}_{3}$ profile exibits a local maximum corresponding to the cloud base location $(16-17 \mathrm{~km})$, where sublimation of the transported NAT particles occurs. The formation of the largest NAT particles $(5-10 \mu \mathrm{m}$ radius) requires that nucleation occurs only at low particle densities with an upper limit of about $10^{-2} \mathrm{~cm}^{-3}$. Such large particle sizes are consistent with the large NAT particle mode observed during SOLVE (Fahey et al., 2001), with the low number of initial NAT nuclei being the key to the "NAT-rock" production. When the NAT nuclei number densities in the Jensen et al. simulation are increased, this effectively spreads the available $\mathrm{HNO}_{3}$ over more NAT particles, which consequently cannot grow to sizes promoting rapid sedimentation. The resulting NAT condensation/renitrification cycle takes place over a limited vertical height range without producing permanent denitrification. Longer simulated lifetimes of PSCs produced more severe denitrification, as also determined by other studies (Toon et al., 1990; Tabazadeh et al., 2000), enabling a corresponding increase in downward transport of $\mathrm{HNO}_{3}$ and renitrification below a cloud base located at lower altitudes. In the presence of rapidly sedimenting solid nitric acid hydrate particles the gas-phase $\mathrm{HNO}_{3}$ does not necessarily reach an equilibrium state (Lowe and MacKenzie, 2008). Vertical redistribution of $\mathrm{HNO}_{3}$ by evaporation of sedimenting PSCs has been observed in the Arctic (Hübler et al., 1990; Arnold et al., 1998; Irie et al., 2001; Kondo et al., 2000; Koike et al., 2002; Dibb et al., 2006; Jin et al., 2006) and the Antarctic (Hofmann and Deshler, 1991).

\section{Instruments, datasets and analysis}

\subsection{A-train satellite constellation}

NASA's Earth Observing System (EOS) Aura satellite, launched on 15 July 2004, is operated in a $98^{\circ}$ inclination sun-synchronous orbit at an altitude of $705 \mathrm{~km}$ with a 13:35 ascending-node time. The Microwave Limb Sounder (MLS) (Waters et al., 2006) is one of four instruments on board the Aura platform, whose main mission objectives are studying ozone, air quality and climate (Schoeberl et al., 2006). MLS detects thermal microwave emission from the
Earth's limb and retrieves vertical profiles of atmospheric temperature and composition in the vertical range $8-90 \mathrm{~km}$ (Livesey et al., 2006).

Aura is a key satellite in the NASA/ESA afternoon "Atrain" satellite constellation and flies in formation with the Aqua, CloudSat, CALIPSO and PARASOL (Polarization and Anisotropy of Reflectances for Atmospheric Sciences coupled with Observations from a Lidar) satellites, among others. CloudSat and CALIPSO were carried into orbit in April 2006 on the same launch vehicle and are operated to maintain very close positioning with respect to Aqua to permit synergistic cloud measurements with Aqua MODIS (Moderate Resolution Imaging Spectroradiometer). In the initial configuration, Aura lagged the lead satellite Aqua by about $15 \mathrm{~min}$, and the CloudSat/CALIPSO ground-track and the MLS limb-track were separated spatially by about $200 \mathrm{~km}$ and temporally by 7-8 min. From November 2007 to April 2008, Aura was gradually re-positioned further forward in the A-train and MLS, CloudSat and CALIPSO have since maintained much closer tracking, with spatial and temporal differences generally better than $10 \mathrm{~km}$ and $30 \mathrm{~s}$, respectively. The $98^{\circ}$ inclination of the A-train satellite orbit results in a closest approach of $8^{\circ}$ to the poles, and the $705 \mathrm{~km}$ altitude and $98 \mathrm{~min}$ orbital period result in a global coverage consisting of 14.5 orbits per day and a 233 orbit (16 day) repeat cycle.

\subsection{Aura Microwave Limb Sounder}

The Aura Microwave Limb Sounder is an advanced successor to the MLS instrument on UARS and measures thermal emission at millimeter and sub-millimeter wavelengths using seven radiometers to cover five broad spectral regions. The radiometric and spectral performance of the MLS instrument is described in detail by Jarnot et al. (2006) for the GHz radiometers. The MLS line-of-sight is in the forward direction of the Aura spacecraft flight track. The Earth's limb is scanned from the surface to $90 \mathrm{~km}$ every $24.7 \mathrm{~s}$, giving 240 scans per orbit spaced at $1.5^{\circ}(165 \mathrm{~km})$ intervals, with a total of $\sim 3500$ vertical profiles per day and a nearly global latitude coverage from $82^{\circ} \mathrm{S}-82^{\circ} \mathrm{N}$. The MLS limb radiance measurements are inverted using a 2-D optimal estimation retrieval (Livesey et al., 2006) to yield atmospheric profiles of temperature, geopotential height, ozone, humidity and other trace gases. The Aura MLS version 2.2 (v2.2) products have been validated comprehensively (Livesey et al., 2008), and the entire MLS dataset has since been reprocessed using updated version 3.3 (v3.3) algorithms (Livesey et al., 2011). The MLS v3.3 data have significant improvements for a number of species, including the $\mathrm{H}_{2} \mathrm{O}, \mathrm{HNO}_{3}, \mathrm{O}_{3}$, and $\mathrm{ClO}$ data products. Correlative measurement comparisons show a finescale oscillation in the $\mathrm{v} 2.2 \mathrm{H}_{2} \mathrm{O}$ retrievals, and this retrieval artefact has been eliminated in the v3.3 retrievals, along with improvements in negative biases seen in the $\mathrm{v} 2.2$ retrievals of $\mathrm{HNO}_{3}$ and $\mathrm{ClO}$. Typical single-profile precisions and spatial 
Table 1. Resolution and precision of selected MLS v3.3 data products.

\begin{tabular}{lcrrl}
\hline Species & Vert. Res. $(\mathrm{km})$ & Horiz. Res. $(\mathrm{km})$ & Precision & Validation Ref. \\
\hline Temperature & $3.5-5.2$ & 165 & $0.6-0.8 \mathrm{~K}$ & Schwartz et al. (2008) \\
$\mathrm{H}_{2} \mathrm{O}$ & $3.1-3.5$ & $180-290$ & $4-15 \%$ & Read et al. (2007), Lambert et al. (2007) \\
$\mathrm{HNO}_{3}$ & $3.5-5.5$ & $400-550$ & $0.7 \mathrm{ppbv}$ & Santee et al. (2007) \\
\hline
\end{tabular}

resolutions for relevant species are given in Table 1 for the pressure range 100 to $10 \mathrm{hPa}$ (hectopascal).

\subsection{CALIPSO/CALIOP}

The CALIPSO satellite carries the CALIOP dual-wavelength elastic-backscatter instrument (Winker et al., 2009). CALIOP measures vertical profiles of aerosol and cloud backscatter at $532 \mathrm{~nm}$ (polarized) and $1064 \mathrm{~nm}$ with spatial resolutions of $0.03 \mathrm{~km}$ vertical, $0.33 \mathrm{~km}$ along-track and an across-track ground footprint of $\sim 70 \mathrm{~m}$. Multiple scattering effects complicate the analysis of optically thick clouds, but this is not an issue with thin PSCs. The laser is linearly polarized and the total backscatter and perpendicular component are detected at the $532 \mathrm{~nm}$ wavelength. On-board data processing is used to average single-shot samples over specific height ranges. Since March 2009, operation has switched to the second of the two CALIOP lasers after the primary laser showed signs of output level deterioration.

The $532 \mathrm{~nm}$ CALIOP calibration is performed over 11 contiguous $5-\mathrm{km}$ samples in the region $30-34 \mathrm{~km}$, where Rayleigh molecular scattering is assumed. Calibration uncertainties arising from the variable stratospheric sulfate background in this height region are confined to the tropics (Thomason et al., 2008; Vernier et al., 2009). The calibrated data products derived from the three CALIOP channels (Hostetler et al., 2006; Hunt et al., 2009) are attenuated volume backscatter coefficients $\left(\mathrm{km}^{-1} \mathrm{sr}^{-1}\right)$ as a function of height, $z$.

\subsubsection{Lidar data analysis}

We have processed the high resolution CALIOP v3.01 Level $1 \mathrm{~B}$ profile data product by averaging onto a grid with a uniform $5 \mathrm{~km}$ horizontal resolution and a vertical resolution of $90 \mathrm{~m}, 180 \mathrm{~m}, 540 \mathrm{~m}$ and $900 \mathrm{~m}$ for height ranges of $-0.5-$ $8.2 \mathrm{~km}, 8.2-20.2 \mathrm{~km}, 20.2-30.1 \mathrm{~km}$ and $>30.1 \mathrm{~km}$, respectively. This is the same grid on which Wu et al. (2011) carried out an investigation of the CALIOP day and night backscatter noise to characterize the spatial and temporal variations of the noise bias and standard deviation. Separation of PSC from the aerosol background in the presence of noise is a non-trivial process, and the analysis presented here has been restricted to nighttime CALIOP observations because the daytime measurements are inherently noisier.
The attenuated backscatter can be decomposed into the sum of the volume particulate backscatter, $\beta_{\mathrm{p}}$, and molecular backscatter, $\beta_{\mathrm{m}}$, coefficients multiplied by the product of the two-way atmospheric path transmittances.

$\beta^{\prime}(z)=\left(\beta_{\mathrm{p}}(z)+\beta_{\mathrm{m}}(z)\right) T_{\mathrm{m}, \mathrm{ext}}^{2}(z) T_{\mathrm{O}_{3}, \mathrm{abs}}^{2}(z) T_{\mathrm{p}, \mathrm{ext}}^{2}(z)$

where the subscripts " $\mathrm{p}$ " and " $\mathrm{m}$ " refer to particulate and molecular scattering, respectively. $T_{x}^{2}$ is the two-way path transmittance due to each of molecular extinction, ozone absorption and particulate extinction (Hostetler et al., 2006). The attenuated backscatter is corrected for molecular extinction and ozone absorption by using GEOS-5 temperatures and $\mathrm{MLS}_{3}$ data for the calculation of molecular scattering and atmospheric path transmittances. Full details of the CALIOP molecular scattering calculations are given by Hostetler et al. (2006).

Single wavelength elastic-backscatter measurements lead to an under-determined set of equations, with two unknown quantities (backscatter and extinction) at each altitude and only one measurement (attenuated backscatter). Various methods are used to relate particulate backscatter to volume extinction properties (so-called Lidar ratio): (a) scaling parameterizations, (b) independent measurements of optical depth and (c) optical models of the wavelength dependence of the particle properties (refractive index, shape, size distribution) coupled with Mie scattering (spherical) calculations or non-spherical models (e.g., $T$-matrix methods). Here we employ a correction to the attenuated backscatter for the molecular scattering and ozone absorption only, in effect assuming that $T_{\mathrm{p}}=1.0$. Noel et al. (2009) noted that PSCs are frequently subvisual (optical depth $<0.03$ ) and that optical depths rarely exceed 0.3 . For optical depths below 0.1 , the resulting error in the backscatter is less than $20 \%$. This approach is consistent with the use of the CALIOP data on low opacity PSCs by other investigators (Noel et al., 2008; Pitts et al., 2007, 2009).

\subsubsection{Summary of derived quantities from the lidar}

Total attenuated backscatter is given by the sum of the parallel, $\beta_{\|}$, and perpendicular, $\beta_{\perp}$, components

$\beta_{T}=\beta_{\|}+\beta_{\perp}$ 
The backscatter ratio (backscatter relative to Rayleigh scattering) is given by

$R_{\mathrm{T}}(z)=\frac{\beta_{\mathrm{p}}(z)+\beta_{\mathrm{m}}(z)}{\beta_{\mathrm{m}}(z)}$

Particulate depolarization, $\delta_{\mathrm{p}}$, is obtained from the ratio of the perpendicular to parallel lidar signals

$\delta_{\mathrm{p}}=\left(\frac{R^{\perp}-1}{R^{\|}-1}\right) \delta_{\mathrm{m}} \equiv \frac{\beta_{\perp}-\beta_{\mathrm{m}, \perp}}{\beta_{\|}-\beta_{\mathrm{m}, \|}}$

where $\delta_{\mathrm{m}}$ is the molecular depolarization and is given by Hostetler et al. (2006) as

$\delta_{\mathrm{m}}=\frac{\beta_{\mathrm{m}, \perp}}{\beta_{\mathrm{m}, \|}}=0.00366$

In practice the interpretation of the depolarization ratio is often complicated by the presence of external mixtures of liquid droplets (perfect spheres) and solid (non-spherical) particles along the optical path (Daneva et al., 2003).

A measure of the bulk particle size can be obtained from the color ratio (ratio of the backscatter in the 1064-nm and 532-nm channels), with larger values indicating larger particle sizes, but its use is limited to scattering conditions leading to significant return signals from the noisier $1064 \mathrm{~nm}$ channel. We use only the CALIOP 532-nm data in the current analysis.

\subsubsection{CALIOP PSC detection}

We apply a spike filter to the CALIOP lidar data to remove the space environment radiation-induced noise events that are typically seen at isolated locations (Pitts et al., 2009). The method consists of running a 5-point horizontal filter through the along-track backscatter data and checking the central point values at the along-track location index, $i,\left(R_{\mathrm{T}_{i}}, \beta_{\perp_{i}}\right)$ against the mean values for the four outer location indices, $\left(\bar{R}_{\mathrm{T}_{i}}=\left(R_{\mathrm{T}_{i-2}}+R_{\mathrm{T}_{i-1}}+R_{\mathrm{T}_{i+1}}+R_{\mathrm{T}_{i+2}}\right) / 4\right.$, $\left.\bar{\beta}_{\perp_{i}}=\left(\beta_{\perp_{i-2}}+\beta_{\perp_{i-1}}+\beta_{\perp_{i+1}}+\beta_{\perp_{i+2}}\right) / 4\right)$. For the total backscatter signal, a spike is detected at location, $i$, and the data replaced with a missing value flag for the condition $R_{\mathrm{T}_{i}}>9$ and $\bar{R}_{\mathrm{T}_{i}}<3$. Similarly, for the perpendicular signal, a spike is detected and the data replaced with a missing value flag for the condition $\beta_{\perp_{i}}>1 \times 10^{-4} \mathrm{~km}^{-1} \mathrm{sr}^{-1}$ and $\bar{\beta}_{\perp_{i}}<1 \times 10^{-5} \mathrm{~km}^{-1} \mathrm{sr}^{-1}$. The filter is used only in rejecting noise spikes and the resulting cleaned data are mapped onto the regular analysis grid $(50 \mathrm{~km}$ by $0.5 \mathrm{~km})$ using a Kalman smoothing technique (Rodgers, 2000). The non-uniform vertical resolution is taken into account by weighting according to the measurement integration time.

There is considerable noise in the backscatter measurements and variation due to background aerosols. The total and perpendicular backscatter thresholds, which determine the PSC detection limits, have been adapted for the different noise characteristics of the data gridding used in this investigation. Detection thresholds were determined from statistical considerations of the CALIOP measurements of the aerosol background at temperatures above expected PSC formation conditions $(>205 \mathrm{~K})$. We have found that fixed minimum thresholds for the total backscatter ratio and perpendicular backscatter are acceptable for the height range used in this investigation. Additionally, we have imposed tests for spatial correlation, which require detections above the thresholds for five out of nine locations defined by a 3 by 3 grid box centered on the PSC location. A positive detection requires either: (i) a spatially correlated total backscatter ratio $\left(R_{\mathrm{T}} \geq\right.$ 1.25 ) or (ii) a spatially correlated perpendicular backscatter $\left(\beta_{\perp} \geq 2.5 \times 10^{-6} \mathrm{~km}^{-1} \mathrm{sr}^{-1}\right)$ combined with a lower threshold for the total backscatter ratio $\left(R_{\mathrm{T}} \geq 1.15\right)$. The false positive detection rate is determined to be less than $0.1 \%$, based on analysis of data selected for $T-T_{\mathrm{ICE}}>10 \mathrm{~K}$, during early May 2008 when no PSCs are expected to be present.

\subsection{Combined CALIOP/MLS satellite data analysis}

Data from each orbit transect of the Antarctic polar regions were assembled into height vs. along-track cross-sections of colocated MLS species and CALIOP data with horizontal and vertical grid spacings of 50 and $0.5 \mathrm{~km}$, respectively, as shown in Fig. 1. Geometric heights for MLS are determined from the retrieved geopotential heights. These vertical cross-sections have proven to be a very useful means of examining an extremely large data set and identifying potential anomalous events and cases for further investigation. Derived information such as the backscatter vs. depolarization distribution is also produced. The equilibrium composition of STS is calculated from the parameterization of the thermodynamic model by Carlsaw et al. (1995) and vapor pressures calculated from the expressions given by Hanson and Mauersberger (1988) for NAT and Murphy and Koop (2005) for ice. The majority of the MLS species, including $\mathrm{HNO}_{3}$, are retrieved at a lower vertical resolution (6 levels per decade in pressure) than are temperature, $\mathrm{H}_{2} \mathrm{O}$ and $\mathrm{O}_{3}$ (each at 12 levels per decade). The MLS data are interpolated to the analysis grid with an effective over-sampling of a factor of three in the horizontal and a factor of three (five) for the higher (lower) vertical-resolution species.

\subsection{MIPAS}

The MIPAS instrument (Fischer et al., 2008) was launched in March 2002 on the ESA Environment Satellite (EnviSat). MIPAS is an infrared Fourier transform spectrometer for the detection of limb emission spectra which, through azimuth scanning, provides global coverage from $87.5^{\circ} \mathrm{S}$ to $89.3^{\circ} \mathrm{N}$. The instrument field-of-view is $30 \mathrm{~km}$ across-track and $3 \mathrm{~km}$ in the vertical. The horizontal along-track sampling is $\sim 500 \mathrm{~km}$. We have used the MIPAS v4.67 Level 1B radiance data and applied a correction to the engineering tangent altitude registration for a reported offset of $1.5 \mathrm{~km}$ at the South Pole. 


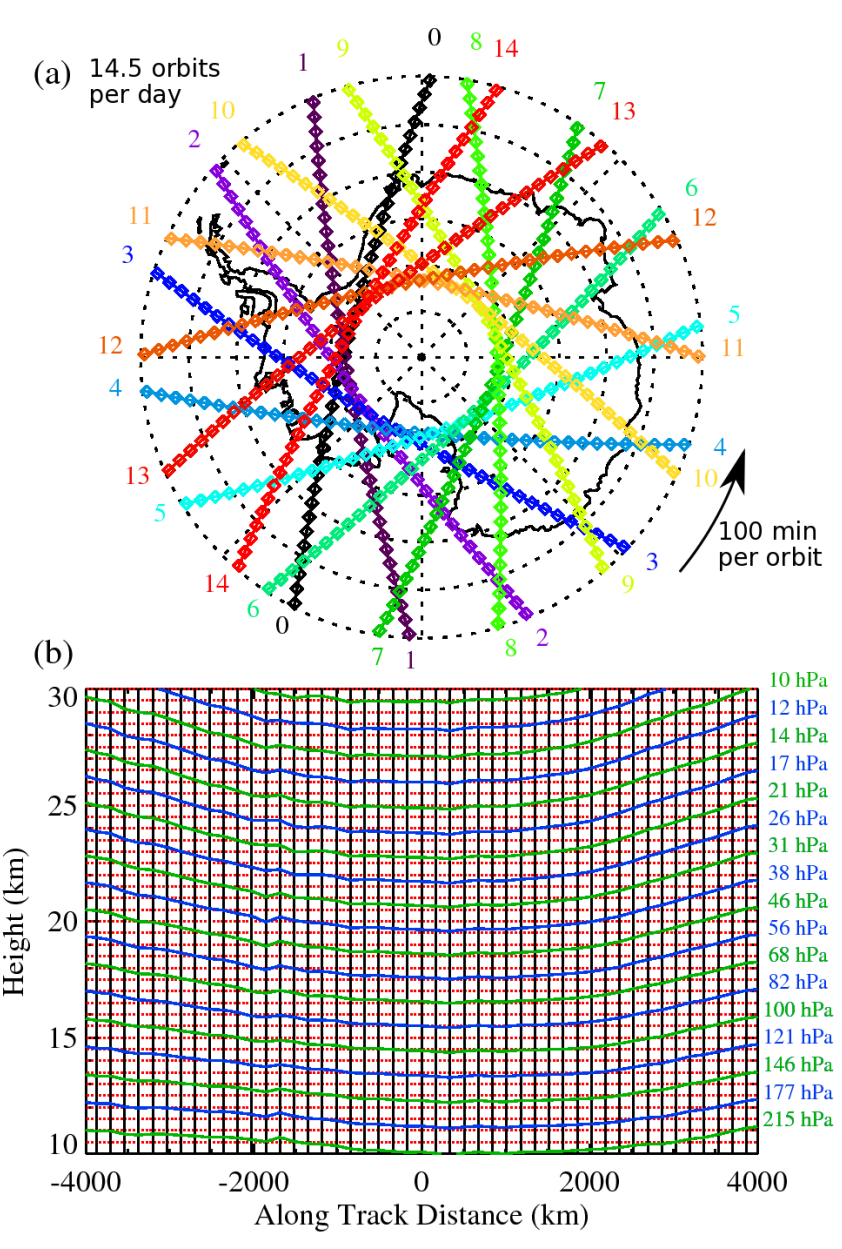

Fig. 1. (a) Example of a complete day of orbit tracks over Antarctica, with different colors identifying each orbit. The along-track colocation origin is defined as the closest approach of the orbit track to the South Pole. The satellites cover a distance of $1800 \mathrm{~km}$ in $4 \mathrm{~min}$, and during this time MLS makes 11 limb scans and CALIOP fires 4800 laser shots. (b) Height vs. along-track data analysis grid (red dots) with corresponding pressure levels indicated on the right vertical axis. The horizontal and vertical spacings are $50 \mathrm{~km}$ and $0.5 \mathrm{~km}$, respectively. The majority of the MLS species, including $\mathrm{HNO}_{3}$, are retrieved at a lower vertical resolution (6 levels per decade in pressure, green lines) than are temperature, $\mathrm{H}_{2} \mathrm{O}$ and $\mathrm{O}_{3}$ (12 levels per decade, blue and green lines).

We have used retrievals of cloud top height (Hurley et al., 2011) and the color-ratio PSC type discrimination developed by Spang et al. (2004) and Höpfner et al. (2006b) to supplement the measurements from CALIOP. MIPAS PSC detection relies on a composition independent cloud-index parameter (CI), which is inversely related to cloud optical thickness, and defined as the ratio of the mean spectral intensities in the wavenumber ranges $788.2-796.25 \mathrm{~cm}^{-1}$ and $832.3-834.4 \mathrm{~cm}^{-1}$. The threshold used for PSC detection is CI $<4.5$ (Spang and Remedios, 2003), and calculations by Höpfner et al. (2006b) indicate this corresponds to a vol- ume density detection limit of $0.2-0.4 \mu \mathrm{m}^{3} \mathrm{~cm}^{-3}$. A second radiance ratio (referred to here as the NAT spectral parameter, RR), is used to detect NAT-containing clouds from a distinctive spectral feature near $820 \mathrm{~cm}^{-1}$, and is defined as the ratio of the mean spectral intensities in the wavenumber ranges $819.0-821.0 \mathrm{~cm}^{-1}$ and $788.2-796.25 \mathrm{~cm}^{-1}$. The NAT $820 \mathrm{~cm}^{-1}$ spectral feature flattens as the mean particle radius increases beyond 2-3 $\mu \mathrm{m}$ (Höpfner et al., 2006b), resulting in an inability to discriminate larger NAT particles from STS.

\subsection{Aqua AIRS}

The AIRS instrument (Aumann et al., 2003), launched in May 2002 on the Aqua satellite, is a multispectral infrared imager with a horizontal resolution of $13.5 \mathrm{~km}$ at the nadir. AIRS measurement scene granules are assembled from the data in a $\sim 1650 \mathrm{~km}$ wide swath accumulated over a time period of $6 \mathrm{~min}$ and consist of 135 contiguous across-track scan sweeps each containing 90 ground footprints with a $1.1^{\circ}$ field-of-view. The horizontal resolution is $13.5 \mathrm{~km}$ at nadir increasing to $41 \mathrm{~km}$ by $21.4 \mathrm{~km}$ at the scan extremes. We have analyzed the AIRS v5.0 Level 1b radiances using the methods of Eckermann et al. (2009) to detect gravity waves. After converting selected radiance channels to form a brightness temperature product and horizontal filtering and smoothing to reduce noise, small-scale residuals down to a wavelength of $\sim 300 \mathrm{~km}$ may be detected (Eckermann et al., 2009). The vertical weighting functions of the AIRS instrument are quite broad in height $(\sim 10 \mathrm{~km})$ and unable to resolve short vertical wavelength features (Hoffmann and Alexander, 2009). However, comparisons with MLS temperatures for gravity wave events (not shown) indicate that AIRS has some sensitivity to vertical wavelengths as short as $7 \mathrm{~km}$. We have analyzed the AIRS radiance brightness temperature perturbations for selected channels with peak responses near 80 and $40 \mathrm{hPa}$ as determined by Eckermann et al. (2009).

We calculate the standard deviation about the background mean brightness temperature and accumulate the statistics of the perturbation amplitudes from each scene for each complete day of observations. We define a perturbation greater than 4.5 standard deviations above (or below) the background mean to be a significant detection and therefore disregard the wave phase in order to combine data obtained at different AIRS measurement times throughout the whole day, which allows us to gauge the evolution of the gravity wave activity in terms of the locations, intensities and number of detections for each day.

\subsection{Collocated meteorological data}

We use the Goddard Earth Observing System Data Assimilation System (GEOS-5 DAS) 6-hourly gridded data products (Rienecker et al., 2008) of temperatures and winds supplied on a 540 by 361 longitude-latitude grid. The 6-hourly temperature data are also interpolated in location and time to the 
analysis grid shown in Fig. 1. Lagrangian tracking of air parcels is a well established technique for selecting relevant spatially correlated time-ordered measurement samples from satellite data sets (e.g., Danilin et al., 2002). The technique allows separation of chemical changes from dynamical processes by linking together a series of measurements of the same air parcel at different locations and times. In this analysis we use isentropic Lagrangian trajectories to determine the synoptic temperature-time history of air parcels and to establish their provenance. We interpolate the GEOS-5 data products onto a potential temperature grid with a spacing of $25 \mathrm{~K}$ to facilitate the trajectory analyses. A standard fourthorder Runge-Kutta integration with a time step of $15 \mathrm{~min}$ is used to update the trajectory coordinates.

\subsection{Theoretical modeling of liquid-solid PSC mixtures}

We have carried out microphysical and optical calculations for STS and NAT particle distributions for equilibrium conditions and representative non-equilibrium STS-NAT mixtures following the methodology used by Pitts et al. (2009) in deriving the CALIOP PSC classification algorithm. This is based on in situ observations of particle size and number density (Dye et al., 1992; Fahey et al., 2001; Northway et al., 2002) and used a fixed total particle number density, $N_{\text {TOT }}=10 \mathrm{~cm}^{-3}$. Pitts et al. (2009) displayed the results of their calculations in a form suitable for PSC classification, i.e., depolarization vs. normalized backscatter ratio. In this investigation we are also interested in the temperature variation of the total backscatter, depolarization and gas-phase uptake of $\mathrm{HNO}_{3}$ for the various STS-NAT mixtures.

The calculations used a representative atmospheric pressure of $46 \mathrm{hPa}$, with a low background $\mathrm{H}_{2} \mathrm{SO}_{4}$ value of $0.1 \mathrm{ppbv}$ (parts per billion by volume) (Tsias et al., 1999), 12 ppbv for $\mathrm{HNO}_{3}$ and 5 ppmv (parts per million by volume) for $\mathrm{H}_{2} \mathrm{O}$. Although apparently limited to a particular atmospheric pressure, the results are applicable to a wider range of total pressures, as noted by Biele et al. (2001) and Drdla et al. (2003), through a transformation of the temperature coordinate to $T-T_{\text {ICE }}$, which removes variations due to $\mathrm{H}_{2} \mathrm{O}$ partial pressure, or $T-T_{\mathrm{NAT}}$, which additionally removes the smaller variations due to $\mathrm{HNO}_{3}$ partial pressure.

PSC particle distributions were parameterized according to the same scenarios given by Pitts et al. (2009). For the STS only scenario: the particle size distribution assumed is lognormal with standard deviation, $\sigma=1.6$, and number density, $N_{\mathrm{STS}}=10 \mathrm{~cm}^{-3}$, and the mode radius derived from the equilibrium condensed STS volume obtained from the Carlsaw et al. (1995) model for temperatures between $196 \mathrm{~K}$ and $3 \mathrm{~K}$ below the frost point. For STS-NAT mixtures: an inverse power law NAT particle distribution is assumed with fixed effective variance of 0.1 (Liu and Mishchenko, 2001), effective radii varying from 0.5 to $7 \mu \mathrm{m}$ and NAT number densities varying from $10^{-4}$ to $1 \mathrm{~cm}^{-3}$. The NAT volume is constrained to not exceed the equilibrium NAT volume.
Co-existing STS particles are adjusted such that their number density is reduced to preserve the total number density, i.e., $N_{\mathrm{STS}}=N_{\mathrm{TOT}}-N_{\mathrm{NAT}}$ and the residual gas-phase $\mathrm{HNO}_{3}$ is adjusted for the amount condensed in the NAT particles.

Optical properties were calculated using Mie theory for liquid spherical particles and the $T$-matrix method for solid NAT (Mishchenko and Travis, 1998). There is no simple depolarization dependency on the particle shape (Liu and Mishchenko, 2001; Reichardt et al., 2002), and a variety of shapes are consistent with lidar observations (Reichardt et al., 2004). Particle shapes for NAT are likely to be physically more complex figures than spheroids or cylinders; however, these are commonly used for the interpretation of lidar measurements:

- Liu and Mishchenko (2001) indicated that lidar measurements of Type Ia PSC particles could be explained either by spheroids (prolate with $e<0.8$ or oblate with $e>1.2$ ) or by cylinders (oblate with diameter-to-length ratios larger than 1.6 or prolate with length-to-diameter ratios larger than 1.4).

- Toon et al. (1990) used a prolate spheroidal shape with $e=0.8$ to approximate cylindrical shapes of trihydrate crystals (length-to-diameter ratios of about 1.22) grown from liquid (Taesler et al., 1975).

- Brooks et al. (2004) found that for comparisons of lidar and in situ measurements in the presence of large NAT particles $(r>5 \mu \mathrm{m})$, the best fit was obtained for a refractive index 1.3-1.6 and an oblate aspect ratio in the range $e=1.5-2.0$.

- Wagner et al. (2005) concluded from comparisons of $T$-matrix calculations with infrared spectral measurements of laboratory-generated homogeneously nucleated NAD crystals with median radii of $\leq 1 \mu \mathrm{m}$ that their shapes were predominantly oblate. Since the nucleation experiments mimicked a potential pathway for the formation of nitric acid hydrates in the atmosphere, they inferred that PSCs could be composed of highly aspherical and possibly platelike (i.e., oblate with $e>5$ ) particles. These large aspect ratios are outside the range investigated for the atmosphere by Liu and Mishchenko (2001) or by Brooks et al. (2004).

We have used the CALIOP PSC classification regions determined by Pitts et al. (2009) which are based on oblate spheroids with an aspect ratio $e=1.2$. However, we have also used prolate spheroidal NAT with $e=0.8$ to illustrate the sensitivity of the perpendicular backscatter coefficient (see Sect. 4.2.1). We explored the uncertainties in particle shape within the limits of computation for the $T$-matrix spheroidal model by performing calculations (not shown) for monodisperse $6-\mu \mathrm{m}$ radius NAT particles with aspect ratios from 0.7 to 1.5 in 0.025 increments. We found a plateau in the depolarization ratio (around $10 \pm 5 \%$ ) in the prolate 


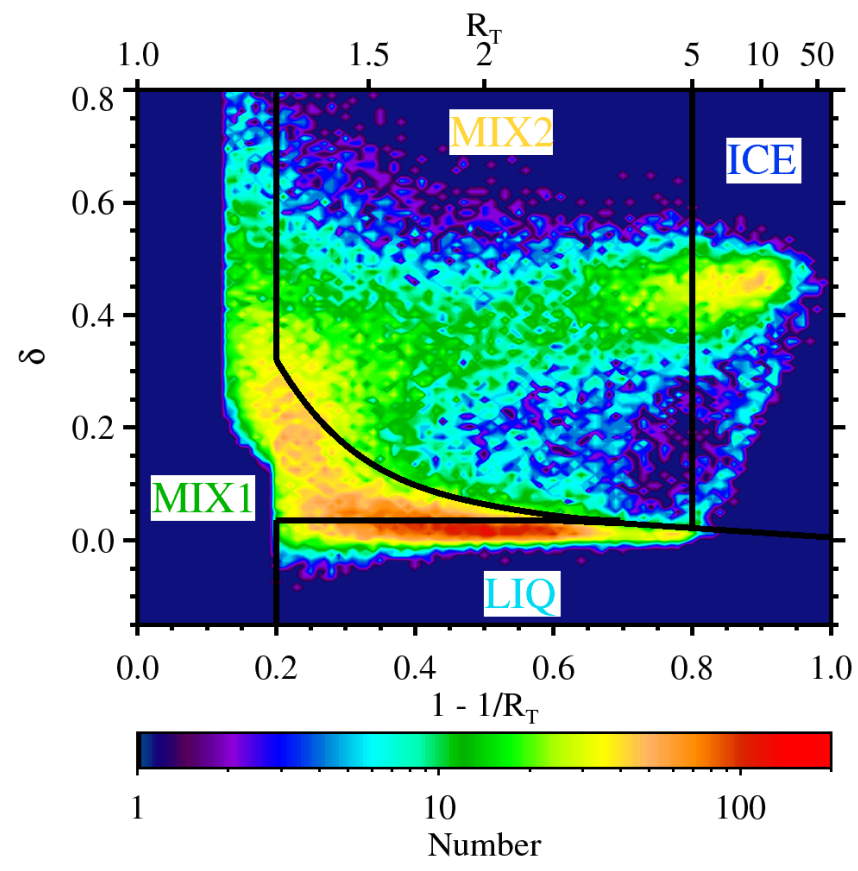

Fig. 2. Distribution of aerosol depolarization vs. normalized backscatter for the period 11-30 June 2008, showing the PSCs identified by CALIOP at an altitude of 20-22 km (near the MLS $32 \mathrm{hPa}$ retrieval level). The color bar indicates the number of observations per bin, with red hues being high and purple hues low. Solid lines mark the boundaries of the CALIOP PSC classification scheme.

range $e=0.70-0.85$ and a higher plateau (around $25 \pm 5 \%$ ) in the oblate range $e=1.2-1.5$. The more nearly spherical particles $(e=0.90-1.10)$ show substantially larger depolarizations (over $60 \%$ ). A similar effect for spheroidal aspect ratios near unity and increasing particle radius was noted by Liu and Mishchenko (2001), who also commented that backscatter characteristics are more aspect-ratio dependent for spheroids than for cylinders. Real refractive indices at $532 \mathrm{~nm}$ were assumed to be 1.43 for STS, and 1.50 for NAT, with zero imaginary refractive indices for both particle types.

\section{Results}

\subsection{CALIOP PSC classification}

The CALIOP PSC type classification algorithm developed by Pitts et al. (2009) categorizes the total and perpendicular lidar backscatter signals into one of four statistical composition classes based on a depolarization and inverse backscatter relation. PSC classification takes place after the PSC detection stage discussed in Sect. 3.3.3.

\subsubsection{Backscatter and depolarization properties}

Figure 2 shows an example of the depolarization vs. normalized backscatter distribution compiled over a 20-day pe- riod 11-30 June 2008, at $20-22 \mathrm{~km}$ and divided into four composition classes MIX1, MIX2, ICE and LIQ. Here, LIQ corresponds to the STS class of Pitts et al. (2009), consisting mainly of liquid particles that lie along the axis of near-zero depolarization. STS-NAT mixtures are separated by the MIX1/MIX2 boundary, which marks a transition between lower and higher NAT number/volume densities, with the MIX1 (MIX2) class populating the lower (higher) division. We have imposed an additional constraint $\left(\beta_{\perp} \geq\right.$ $2.5 \times 10^{-6} \mathrm{~km}^{-1} \mathrm{sr}^{-1}$ ) on the MIX1 class to accept only PSCs which have significant perpendicular backscatter. This attempts to compensate for the increased noise on the depolarization ratio at low backscatter by placing STS-NAT mixtures dominated by liquid particles into the LIQ class and reserving the MIX1 class for PSCs with a significant nonspherical solid component. The ICE class marks the STS-ice mixtures detected with the highest backscatter ratios.

Pitts et al. (2009) demonstrated that all PSC types are represented at this time of year and observed a mid-June correlation between the increase in the frequency of CALIOP detection of NAT PSC mixtures and a strong decrease observed in the MLS gas-phase $\mathrm{HNO}_{3}$. MIX1 was found to be more prevalent at lower altitudes than MIX2, and this is reflected here in the smaller number of detections of MIX1 compared to MIX2 at 20-22 km. The percentage occurrence for each PSC type detected is LIQ 24\%, MIX1 $28 \%$, MIX2 $40 \%$, and ICE $8 \%$.

\subsubsection{Temperature distribution of PSC types}

The temperature distribution of the detected PSC types during 11-30 June 2008 at 20-22 km is shown in Fig. 3 as a fraction of the number of observations in each temperature bin. The fraction of detections of the MIX1 and MIX2 types starts to increase at $T_{\mathrm{ICE}}+8 \mathrm{~K}$ and reach broad maxima of 0.15 for MIX1 and 0.20 for MIX2 near $T_{\mathrm{ICE}}+3 \mathrm{~K}$. For the LIQ class, the fraction starts to increase at $T_{\mathrm{ICE}}+4 \mathrm{~K}$ and reaches a well defined peak of 0.35 at the ice frost point. There is a rapid increase in detections of MIX2 and ICE types within $1 \mathrm{~K}$ of the frost point. The fraction of all PSCs detected increases rapidly below $T_{\mathrm{ICE}}+1.5 \mathrm{~K}$ and reaches 0.6 at the ice frost point and over 0.9 for $T_{\mathrm{ICE}}-1 \mathrm{~K}$. Occasionally ice is misidentified by the classification scheme because of the conservative placement of the MIX2/ICE boundary, as also noted by Höpfner et al. (2009). For example, this can occur in regions of MIX2 PSCs (not shown), but may only become apparent in situations where locations are over the ocean and remote from any topographic features and the temperatures are several kelvin above the frost point.

\subsection{Constraints provided by simultaneous observations of backscatter, depolarization and gas-phase $\mathrm{HNO}_{3}$}

NAT particles can significantly deplete the gas-phase $\mathrm{HNO}_{3}$ abundance even for quite low particle number densities (less 


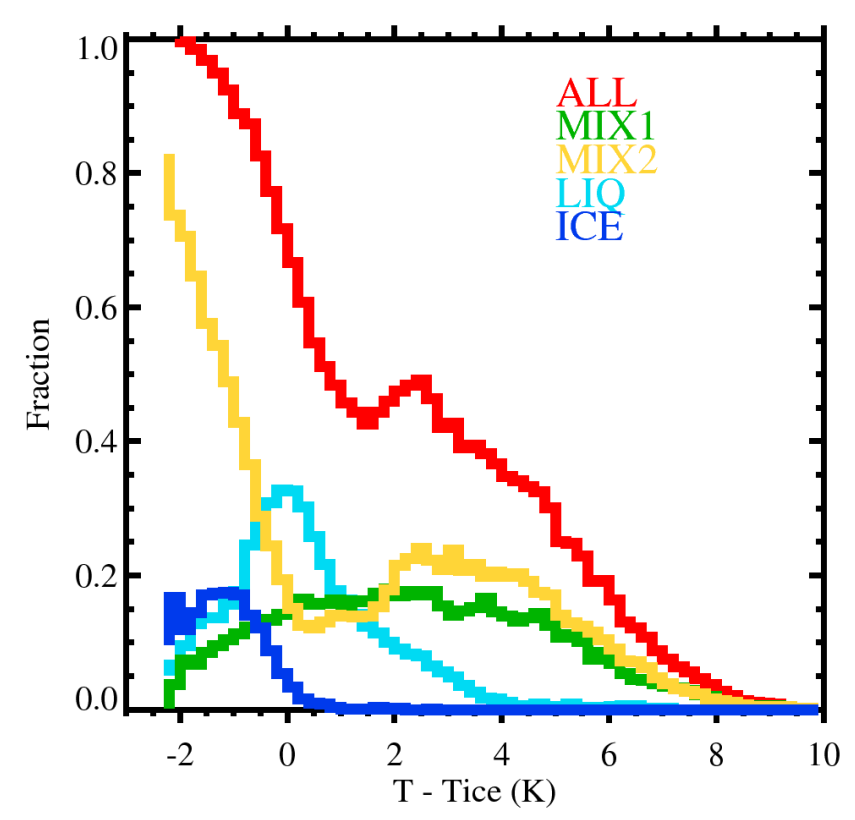

Fig. 3. Fraction of positive PSC detections compared to all CALIOP observations as a function of temperature relative to the ice frost point for the PSC types classified in Fig. 2, MIX1 (green line), MIX2 (yellow line) LIQ (cyan line) and ICE (blue line). The sum of the detections of all four PSC types (red line) is also indicated.

than $10^{-3} \mathrm{~cm}^{-3}$ ) if their effective radii are sufficiently large (greater than about $4 \mu \mathrm{m}$ ). However, the corresponding effects on the optical properties of the backscatter from the solid particles can be rather harder to detect. Here we show that the presence of NAT particles with low number densities may fall below the detection threshold of CALIOP even for the largest particle sizes. At temperatures where STS forms, the situation becomes even more difficult for identifying the solid hydrate component since the liquid particles begin to dominate the parallel backscatter return signal (Daneva et al., 2003). These issues have been discussed by Biele et al. (2001), who showed that the perpendicular backscatter is better for the purposes of detecting very low concentrations of the solid particle component in mixed phase PSCs than the aerosol depolarization. Figure 4 shows the calculated total backscatter, perpendicular backscatter and the fractional gas-phase $\mathrm{HNO}_{3}$ as a function of temperature for STS-NAT mixtures with NAT effective radii of $1 \mu \mathrm{m}$ and $6 \mu \mathrm{m}$. For a given NAT particle number density, the uptake of $\mathrm{HNO}_{3}$ with decreasing temperature follows the NAT equilibrium curve until the saturation point is reached, at which time the equilibrium volume of condensed $\mathrm{HNO}_{3}$ becomes equal to the volume in the assumed NAT distribution. In this model no further uptake of $\mathrm{HNO}_{3}$ from the gas phase occurs until temperatures decrease sufficiently to allow the formation and growth of STS particles and the NAT particles cannot be larger than their equilibrium size. The equilibrium curves for NAT are shown for comparison in Fig. 4 as dashed lines.

\subsubsection{Theoretical limits of detection for PSCs and $\mathrm{HNO}_{3}$ depletion}

Two contrasting scenarios are highlighted in Fig. 4, both causing significant $\mathrm{HNO}_{3}$ depletion, and relevant for the detection of NAT under conditions of small particles/large number densities (associated with fast cooling of mountain waves) and large particles/low number densities (associated with slow synoptic cooling) (Hu et al., 2002). As shown in Fig. 4a, c, e, at temperatures of $4-5 \mathrm{~K}$ above the frost-point, a NAT particle distribution with a small effective radius of $1 \mu \mathrm{m}$ and a high number density of $0.5 \mathrm{~cm}^{-3}$ sequesters about $60 \%$ of the ambient $\mathrm{HNO}_{3}$ (triangle symbol). The total backscatter ratio of $R_{\mathrm{T}}=4.2$ and perpendicular backscatter of $\beta_{\perp}=6 \times 10^{-5} \mathrm{~km}^{-1} \mathrm{sr}^{-1}$ are correspondingly high and well above the detection limits of CALIOP. However, for large NAT particles the expected total backscatter ratio and the perpendicular backscatter can both lie close to or below the thresholds of detection if the NAT number density is sufficiently low, although the resulting $\mathrm{HNO}_{3}$ depletion may be quite easily detectable. As shown in Fig. 4b,d,f, at temperatures of 4-5 K above the frost-point, a NAT particle distribution with an effective radius of $6 \mu \mathrm{m}$ and a number density of $10^{-3} \mathrm{~cm}^{-3}$ sequesters about $25 \%$ of the ambient $\mathrm{HNO}_{3}$ (square symbol). The corresponding total backscatter ratio is quite low $\left(R_{\mathrm{T}}=1.3\right)$, but just above the CALIOP detection threshold. The perpendicular backscatter $\left(\beta_{\perp}=\right.$ $\left.6 \times 10^{-6} \mathrm{~km}^{-1} \mathrm{sr}^{-1}\right)$ is quite sensitive to the actual particle shape (Liu and Mishchenko, 2001) and could lie above or below the CALIOP detection threshold, as shown in Fig. 4d by calculations for the two different assumed aspect ratios of 0.8 and 1.2. This could result in PSCs with large-size NAT particles being classified as non-depolarizing and to be placed in the LIQ class rather than the MIX1 class.

In terms of the NAT number density, the CALIOP detection threshold for a $6-\mu \mathrm{m}$ NAT size distribution is about $4 \times 10^{-4} \mathrm{~cm}^{-3}$, with a corresponding NAT volume density of about $0.3 \mu \mathrm{m}^{3} \mathrm{~cm}^{-3}$, which lies in the $0.2-0.4 \mu \mathrm{m}^{3} \mathrm{~cm}^{-3}$ range of the MIPAS detection limit. The NAT particles sequester about $15 \%$ of the ambient $\mathrm{HNO}_{3}$. For an atmosphere containing $14 \mathrm{ppbv}$ total $\mathrm{HNO}_{3}$, this results in a decrease in the gas phase of about 2 ppbv, which is readily detectable by MLS. Therefore, under such conditions MLS should be able to detect depletion of $\mathrm{HNO}_{3}$ in PSCs which are at or even below the detection limits of CALIOP.

\subsubsection{Model fit to CALIOP and MLS observations}

In principle, gas-phase $\mathrm{HNO}_{3}$ measurements can provide information on the NAT volume density, restricting the range of solutions of size and number density admissible by the backscatter measurements alone, and offering the possibility to use the combined observations from CALIOP and MLS to constrain the probable effective size of NAT particles. However, in practice there are problems in estimating reliably the 

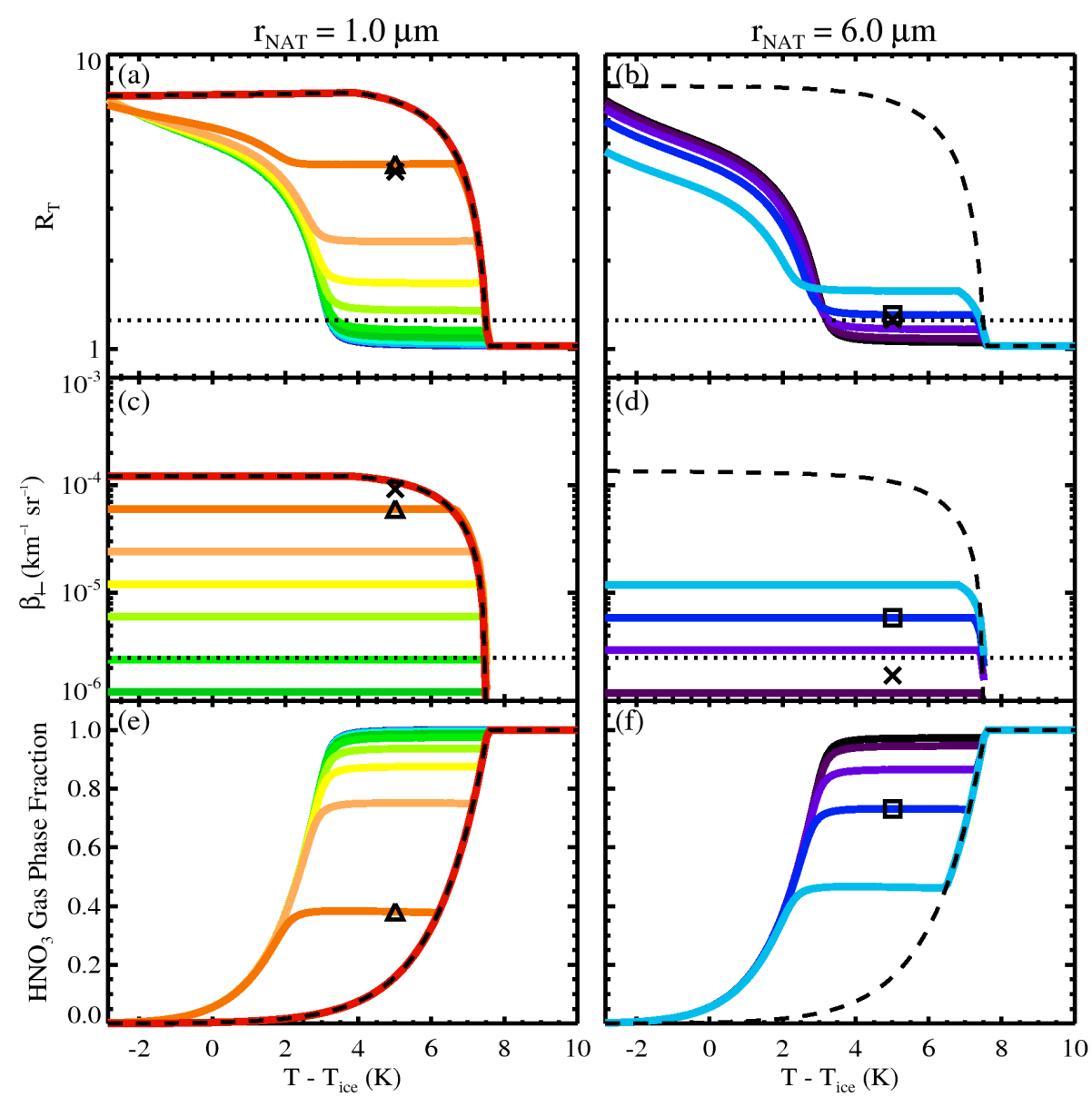

NAT Number densities $\left(\mathrm{cm}^{-3}\right)$ :

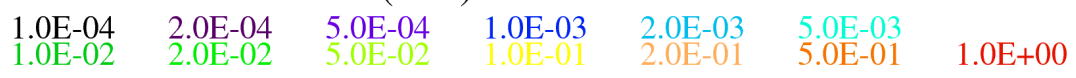

Fig. 4. Temperature variations relative to the frost point of $(\mathbf{a}, \mathbf{b})$ total backscatter ratio, $(\mathbf{c}, \mathbf{d})$ perpendicular backscatter, and $(\mathbf{e}, \mathbf{f})$ total uptake of $\mathrm{HNO}_{3}$ by STS and NAT particles expressed as the fractional remaining gas-phase $\mathrm{HNO}_{3}$, according to the microphysical model of NAT/STS mixtures for different NAT particle number densities in the range $10^{-4}$ to $1 \mathrm{~cm}^{-3}$ and effective radii of $1-\mu \mathrm{m}$ and $6-\mu \mathrm{m}$. Dotted lines indicate CALIOP detection thresholds of (a, b) $R_{\mathrm{T}}=1.25$ and (c, d) $\beta_{\perp}=2.5 \times 10^{-6} \mathrm{~km}^{-1} \mathrm{sr}^{-1}$. Dashed lines show the NAT equilibrium curves. Lines are colored according to the corresponding NAT number densities given at the bottom of the figure. Two contrasting scenarios are highlighted, with both causing significant gas-phase $\mathrm{HNO}_{3}$ depletion: the triangle symbols (a, c, e) denote a small particle/high number density combination and the square symbols (b, d, e) denote large particle/low number density combination. An aspect ratio of 1.2 was used for the $T$-matrix calculations except for the cross symbols, which used a value of 0.8 .

residual gas-phase $\mathrm{HNO}_{3}$ fraction, since although MLS measures the current gas-phase $\mathrm{HNO}_{3}$, the total $\mathrm{HNO}_{3}$ evolves over time through denitrification and renitrification.

We have investigated the use of a simple scheme to obtain a model fit to the observed data in terms of the NAT effective radius, $r_{\mathrm{e}}$, the NAT number density, $N$, and the temperature, $\Delta T=T-T_{\mathrm{ICE}}$, involving the calculation of a $\chi^{2}$ cost-function,

$\chi^{2}\left(r_{\mathrm{e}}, N, \Delta T\right)=\left(\frac{R_{\mathrm{T}}-R_{\mathrm{T}}^{\prime}}{\sigma_{R_{\mathrm{T}}}}\right)^{2}+\left(\frac{\beta_{\perp}-\beta_{\perp}^{\prime}}{\sigma_{\beta_{\perp}}}\right)^{2}+\left(\frac{q-q^{\prime}}{\sigma_{q}}\right)^{2}$

where $R_{\mathrm{T}}$ and $\beta_{\perp}$ are the observed lidar total backscatter ratio and perpendicular backscatter, $q$ is the observed $\mathrm{HNO}_{3}$ gas-phase fraction, and $\sigma$ denotes the measurement uncertainties. The prime symbols denote the predicted quantities, which are calculated for the model as described in Sect. 3.8 as a function of $r_{\mathrm{e}}$ (in $0.1 \mu \mathrm{m}$ steps from 0.5 to $7.0 \mu \mathrm{m}$ ), $N$ (in 37 logarithmic steps from 0.0001 to $1.0 \mathrm{~cm}^{-3}$ ), and $\Delta T$ (in $0.1 \mathrm{~K}$ steps from -3 to $12 \mathrm{~K}$ ). Since we have the temperature from GEOS-5 and $\mathrm{H}_{2} \mathrm{O}$ from MLS, we can calculate $\Delta T$ and therefore determine the best-fit (minimum $\chi^{2}$ ) from a 2-D grid-search over the tabulated $\left(r_{\mathrm{e}}, N\right)$ values. The total ambient $\mathrm{HNO}_{3}$ is the sum of the gas-phase $\mathrm{HNO}_{3}$ and the $\mathrm{HNO}_{3}$ contained in condensed form in the NAT and STS particles. The MLS gas-phase $\mathrm{HNO}_{3}$ combined with an estimated total ambient $\mathrm{HNO}_{3}$ at the PSC location provides 
the gas-phase fraction coordinate in Eq. (6). Precise particle sizing cannot be achieved, but the fit provides a broad categorization allowing to distinguish between PSCs with small size/large number density and large size/small number density. We have used this method as an aid to interpretation of particular measurement cases in Sects. 4.4.5 and 4.5.5.

\subsection{Comparison of observed and modeled uptake of nitric acid in PSCs}

We have analyzed the MLS gas-phase $\mathrm{HNO}_{3}$ variation with temperature for each of the CALIOP PSC composition classes analyzed in Sect. 4.1 for the 20-day period 1130 June 2008, and show the results in Fig. 5a, b as scatter plots, along with the theoretical gas-phase $\mathrm{HNO}_{3}$ uptake curves calculated for thermodynamic equilibrium conditions for STS and NAT at a pressure of $32 \mathrm{hPa}$, assuming $14 \mathrm{ppbv}$ of total $\mathrm{HNO}_{3}, 4.5$ ppmv of $\mathrm{H}_{2} \mathrm{O}$, and 0.1 ppbv of $\mathrm{H}_{2} \mathrm{SO}_{4}$. The observed uptake of $\mathrm{HNO}_{3}$ by the majority of PSCs identified as liquid (LIQ) clearly follows the STS equilibrium curve, but at temperatures about $1 \mathrm{~K}$ lower, although there is a long tail in the distribution extending to temperatures as high as the NAT existence temperature threshold. The $\mathrm{HNO}_{3}$ gas-phase deficit in the presence of PSCs identified as MIX1/MIX2 shows an onset at temperatures slightly higher than the NAT equilibrium curve, and extends to temperatures below the STS equilibrium curve. The temperature region between the STS and NAT equilibrium curves is much more heavily populated by the MIX1/MIX2 types than by the LIQ type. Practically all PSCs identified as ICE are associated with very low residual gas-phase $\mathrm{HNO}_{3}$.

Temperature histograms for the different PSC classifications are shown in Fig. 5c corresponding to ambient $\mathrm{HNO}_{3}$ above 1 ppbv as measured by MLS. This constraint provides better discrimination between the STS and NAT equilibrium curves. Data in the ICE classification are not subject to this constraint. All of the distributions (LIQ, MIX1, MIX2) show PSC types occurring at temperatures above their theoretical equilibrium point and extending down to temperatures below the ice frost point. No significant difference is found between the MIX1 and MIX2 temperature ranges; however, the MIX1 distribution is skewed to lower temperatures and further from the NAT thermodynamic equilibrium than MIX2.

\subsubsection{Evidence for non-equilibrium effects}

The temperatures were transformed according to the appropriate equilibrium curves, with the STS uptake curve applied to the LIQ classification and the NAT curve to the MIX1 and MIX2 classifications, and the resulting temperature histograms are shown in Fig. 5d. The temperature distribution for ICE shows a mean temperature of $1.0 \mathrm{~K}$ below the frost point, with a standard deviation of about $0.5 \mathrm{~K}$, which may be a consequence of more favorable freezing of STS at a depressed temperature (Koop et al., 1998). The transformed
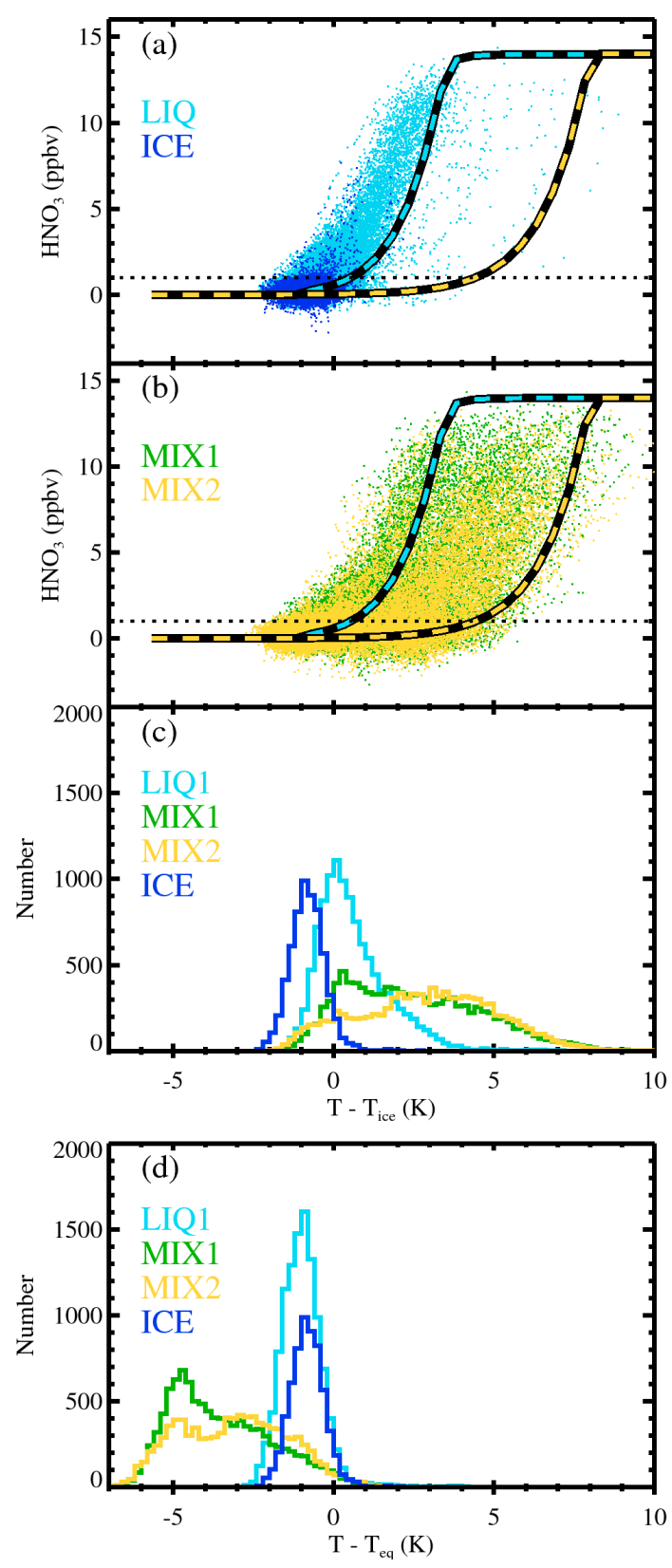

Fig. 5. Composite statistics for 11-30 June 2008, of the MLS gasphase $\mathrm{HNO}_{3}$ variation with temperature in Kelvin corresponding to the PSC types classified in Fig. 2. (a) Scatter plots of $\mathrm{HNO}_{3}$ against the temperature deviation from the frost-point $\left(T-T_{\mathrm{ICE}}\right)$ for PSCs classified as LIQ (cyan) and ICE (blue). (b) As for (a) except for MIX1 (green) and MIX2 (yellow). Equilibrium STS (cyan-black dashed) and NAT (yellow-black dashed) curves show the theoretical uptake of $\mathrm{HNO}_{3}$. (c) Temperature histograms for $\mathrm{HNO}_{3}$ mixing ratios greater than 1 ppbv for each PSC type. Data in the ice classification are not subject to this constraint. (d) Temperatures transformed $\left(T-T_{\mathrm{EQ}}\right)$ according to the equilibrium curves (STS curve applied to the liquid (LIQ) classification and NAT curve to the MIX1 and MIX2 classifications). ICE histogram in (d) remains unchanged from (c) for comparison. 
LIQ distribution mean temperature is $1.1 \mathrm{~K}$ lower than the STS uptake curve, and the standard deviation is $0.7 \mathrm{~K}$. The transformed MIX1 and MIX2 distribution mean temperatures are $\sim 3-4 \mathrm{~K}$ lower than the NAT curve and have larger standard deviations of $1.7 \mathrm{~K}$. Since these MIX1/MIX2 distributions are much broader than for ICE, we infer that the larger spreads are the result of substantial non-equilibrium effects of the solid hydrate particle population, rather than arising from the uncertainties in the GEOS-5 temperature analyses. Likewise, the slightly larger spread for the LIQ distribution compared to ICE may also imply non-equilibrium effects of the STS particle population.

\subsubsection{Measurement uncertainties}

Uncertainties in the temperature analyses and nitric acid measurements contribute to the scatter of the data in Fig. 5. We note that in situ measurements of the aerosol volume density variation with temperature have shown excellent agreement with the calculated STS equilibrium curve (e.g., Drdla et al., 2003) and hence may indicate for the results here a low bias of about $1 \mathrm{~K}$ in the GEOS- 5 temperatures. The mean value of the $\mathrm{HNO}_{3}$ data for the ICE classification is $0.3 \mathrm{ppbv}$, which could imply an actual total uptake of available $\mathrm{HNO}_{3}$ in the presence of ice PSCs since we are unable to rule out such a small positive bias in the MLS $\mathrm{HNO}_{3}$ measurements. The standard deviation is $0.5 \mathrm{ppbv}$, which compares well with the estimated MLS single measurement precision of $0.7 \mathrm{ppbv}$ given in Table 1 .

\subsection{Onset of denitrification in the polar vortex}

\subsubsection{Synoptic-scale cooling and initial $\mathrm{HNO}_{3}$ depletion}

Examination of the GEOS-5 synoptic temperatures over Antarctica in May 2008 indicates that the NAT existence temperature threshold is reached on 4 May. By 21 May temperatures decreased sufficiently to enable the formation of STS in the central vortex region, although the cold pool of air is located beyond the reach of the A-train orbit tracks until 25 May. However, the polar cap region within $8^{\circ}$ of the pole is observed by MIPAS, and from 20 May onwards PSCs are detected there on successive days with increasing frequency. On 19-21 May, small transient decreases are seen in MLS $\mathrm{HNO}_{3}$ near the latitude limit of the Aura orbit tracks, which are also coincident with CALIOP LIQ and MIX1 detections. However, persistent $\mathrm{HNO}_{3}$ depletion is not visible with the MLS instrument until 24 May at $46-32 \mathrm{hPa}$. On the $46 \mathrm{hPa}$ pressure level the $\mathrm{HNO}_{3}$ values within the vortex are about 14 ppbv leading up to 23 May, as shown in Fig. 6a. Following a significant decrease in $\mathrm{HNO}_{3}$ on 24 May, further depletion extends over the Ross Sea as the polar vortex rotates, and by 25 May the lowest $\mathrm{HNO}_{3}$ values are about $7 \mathrm{ppbv}$, constituting removal of $50 \%$ from the gas phase. A concurrent increase of $\mathrm{HNO}_{3}$ on the $68 \mathrm{hPa}$ level is indicated in Fig. $6 \mathrm{~b}$.
Temperatures decrease over 23-25 May, but still remain higher $\left(T-T_{\text {ICE }}>3 \mathrm{~K}\right)$ than required to allow significant STS growth at the observation times of the $\mathrm{HNO}_{3}$ decrease, as shown in Fig. 6c. Therefore, we can rule out the growth of STS alone as responsible for the $\mathrm{HNO}_{3}$ deficit because the uptake of $\mathrm{HNO}_{3}$ by STS is insufficient at these temperatures.

\subsubsection{Quantification of temperature exposure}

GEOS-5 wind analyses were used to calculate isentropic back trajectories for five days starting from each of the MLS measurement locations and observation times. Temperatures were calculated along the trajectories by interpolation from the 6-hourly GEOS-5 analyses with potential temperature levels at $25 \mathrm{~K}$ intervals. We quantify the duration of exposure of an air parcel to low temperatures by defining the temperature threshold exposure (TTE) to be the total integrated time along a trajectory that an air parcel is subject to synopticscale temperatures below the imposed threshold. In Fig. 6d, we show results for a temperature threshold of $5 \mathrm{~K}$ above the ice frost point on the $46 \mathrm{hPa}$ pressure surface and find that the depleted $\mathrm{HNO}_{3}$ region correlates more clearly with the region where TTE $\geq 1.5$ days than with either the region of $\mathrm{T}<T_{\mathrm{NAT}}$ or the region of minimum local temperatures close to $T_{\mathrm{STS}}$ (see Fig. 6c).

\subsubsection{Comparisons of MIPAS and CALIOP PSC observations}

Measurements of the PSC cloud top heights from MIPAS and CALIOP are shown in Fig. 6e, f. MIPAS was operated in a mesosphere observing mode on 25 May that did not sound the lower stratosphere below $18 \mathrm{~km}$. Hence, we have determined a coarse estimate of the cloud top heights on this day from the highest tangent altitude at which the CI parameter indicated a PSC detection. For the standard observing mode days we used the retrieved cloud top heights (Hurley et al., 2011). MIPAS detected PSCs with a range of cloud top heights varying from 18.5 to $22 \mathrm{~km}$, mainly within $8^{\circ}$ of the pole, but with some detections overlapping the region sounded by CALIOP and MLS. CALIOP detected patchy MIX1 PSCs at altitudes varying from 17 to $21 \mathrm{~km}$, but most of the locations associated with low $\mathrm{HNO}_{3}$ measurements are not correlated with PSC detections.

Comparisons between CALIOP and MIPAS matchingpair observations by Höpfner et al. (2009) in 2006-2007 found that overall both instruments detected Antarctic PSCs in common in $85 \%$ of the matches during June-August, but only $60 \%$ in May. In particular, there were considerably more MIPAS-only PSC detections (i.e without corresponding matching detections by CALIOP) in May (25\%), compared to June $(8 \%)$ and July-October $(<3 \%)$. Höpfner et al. (2009) attributed these more frequent detections to the MIPAS limb-viewing geometry, which is oriented towards the pole, and results in sensitivity to PSCs lying poleward 


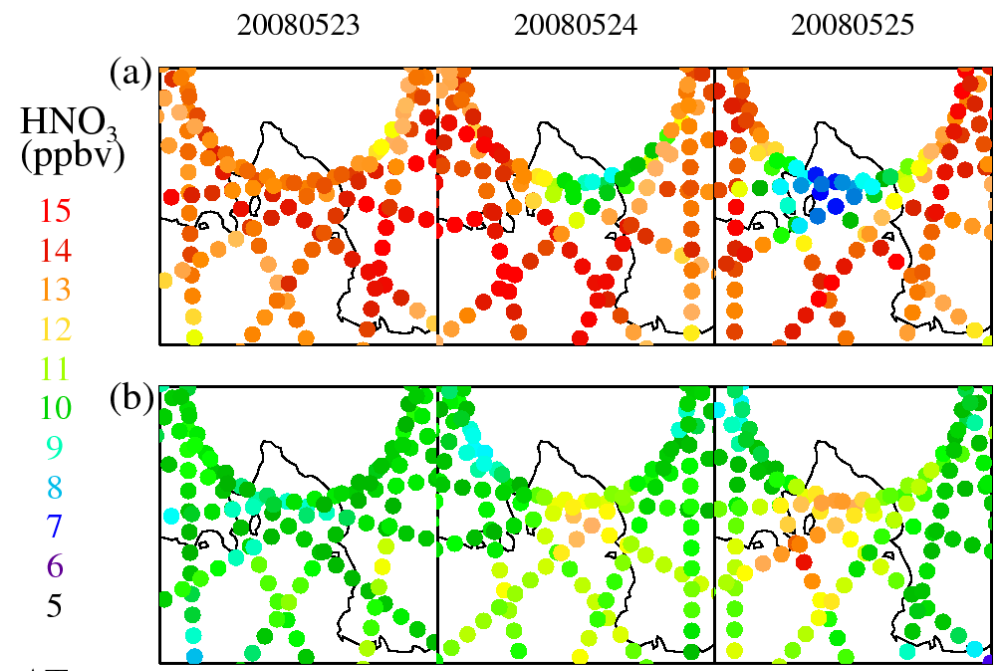

$\Delta \mathrm{T}$
$\mathbf{\mathrm { K }}$
14
12
1
6
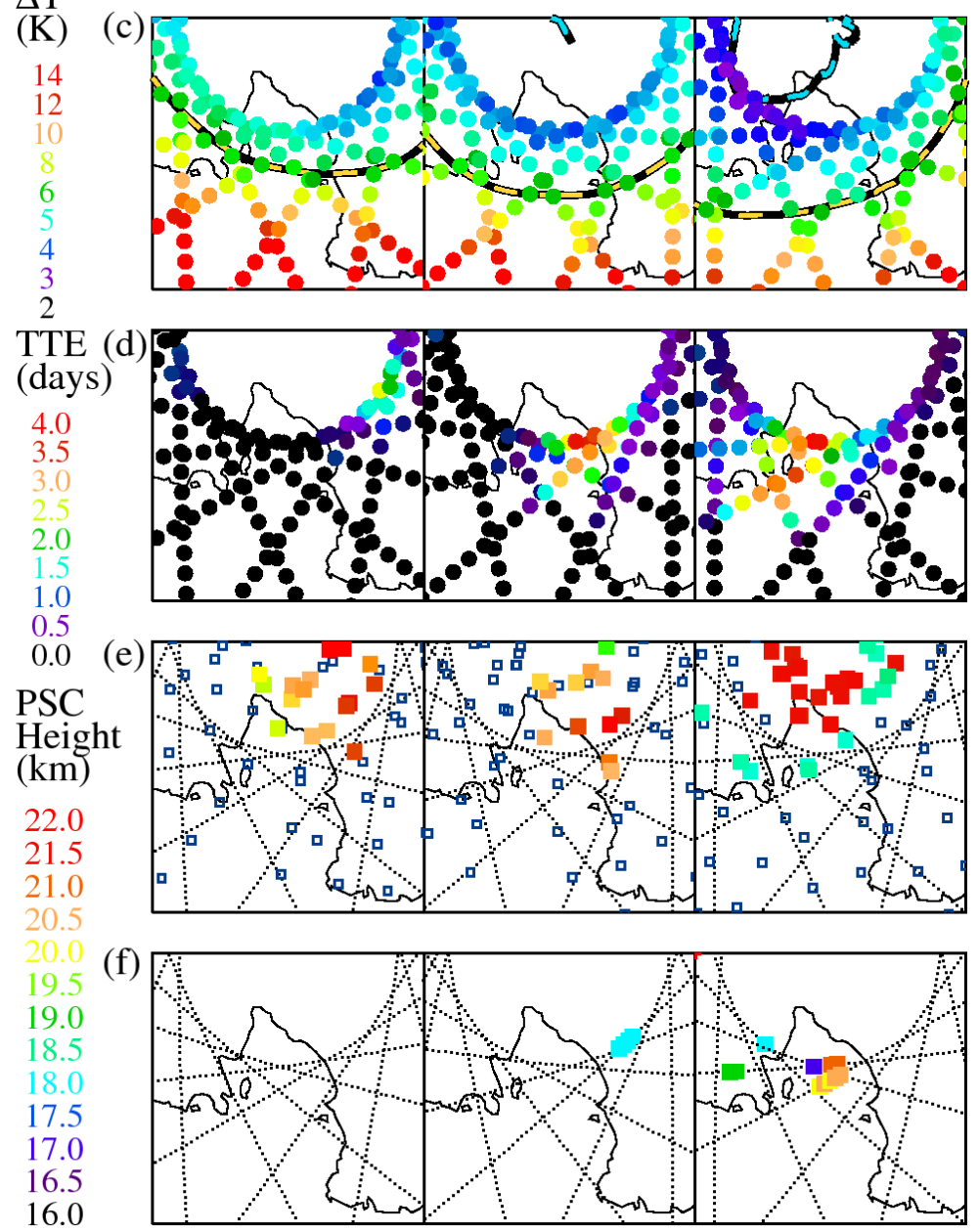

Fig. 6. Orbit plots showing the evolution of $\mathrm{HNO}_{3}$, temperature in Kelvin and PSC detections for selected atmospheric levels over a threeday period 23-25 May 2008. The colors in the orbit maps refer to the colored numerical scales given on the left of the figure. (a) MLS $46 \mathrm{hPa}(20 \mathrm{~km}) \mathrm{HNO}_{3}$. (b) $\mathrm{MLS} 68 \mathrm{hPa}(18 \mathrm{~km}) \mathrm{HNO}_{3}$. (c) Temperature relative to the ice frost point, $\Delta T=T-T_{\mathrm{ICE}}$, on the $46 \mathrm{hPa}$ pressure surface (yellow-black and cyan-black dashed contours indicate the $T_{\mathrm{NAT}}$ threshold and $T_{\mathrm{STS}}$ formation temperatures, respectively). (d) Temperature threshold exposure in days (TTE) for $T-T_{\text {ICE }}<5 \mathrm{~K}$ on the $46 \mathrm{hPa}$ pressure surface. (e) MIPAS PSC top heights (open grey squares are MIPAS observations without PSC detections, and small black dots show the Aura orbit tracks). (f) CALIOP PSC top heights (small black dots are CALIOP observations without PSC detections). 
of the tangent points, but beyond the latitude limit of the CALIOP nadir view. Since PSC formation largely occurs in a region close to the South Pole in May, the MIPAS viewing geometry has a much larger effect on the PSC detection frequency at this time compared to later times when PSCs occur over a more widespread region. Note that the MLS line of sight is in the forward direction along the orbit track and is not oriented towards the pole, therefore the low $\mathrm{HNO}_{3}$ measurements are not biased by the viewing geometry.

\subsubsection{Evidence for large-size NAT particles}

The considerably longer along-track extent of the $\mathrm{HNO}_{3}$ gas-phase deficit must be reconciled with the corresponding below-threshold CALIOP observations. Two possible cases are that the missing gas-phase $\mathrm{HNO}_{3}$ resides mainly in PSC particles with a size distribution/number density combination below the threshold of detection of CALIOP, or that most of the PSC particles have already undergone sedimentation and MLS is detecting the outflow of previously denitrified air. The enhancements of about 2 ppbv in $\mathrm{HNO}_{3}$ on 24-25 May at $68 \mathrm{hPa}$ shown in Fig. $6 \mathrm{~b}$ could be interpreted as the evaporation of solid nitric acid hydrate particles as they sediment from colder regions above and descend into a warmer region with a lower vapor saturation ratio. Vertical wind shear may cause the particles to follow slightly different trajectories as they descend, and so the renitrified layer is not expected to line up directly underneath the depleted layer. If this observation is a manifestation of particle sedimentation, then the implied rate provides a constraint on the particle size. The start of this denitrification event is unlikely to have been much earlier than 20 May, when MIPAS first detected PSCs $(\mathrm{CI}>3.1)$ in the polar cap. Coupled with the MLS observation of a vertical redistribution of $\mathrm{HNO}_{3}$ on 24 May, this gives an upper limit of four days for particle growth. This time-scale is consistent with NAT growth to a radius of over $5 \mu \mathrm{m}$ and a subsequent fall of $4 \mathrm{~km}$ from $32 \mathrm{hPa}(22 \mathrm{~km})$ to $68 \mathrm{hPa}(18 \mathrm{~km})$ (Jensen et al., 2002; Voigt et al., 2005). The MIPAS data during 20-25 May indicate a generally increasing number of clustered PSC detections (3, 10, 16, 23, 13, 31 on consecutive days) with CI values in the range 2.4-4.1 that indicate NAT volume densities above the MIPAS detection limit of $0.2 \mu \mathrm{m}^{3} \mathrm{~cm}^{-3}$. However, even during the earliest observation times, when the NAT particles may be expected to be growing rapidly following nucleation, none of the MIPAS detections show the characteristic NAT spectral signature for particles with radii $<3 \mu \mathrm{m}$, and, as noted in Sect. 3.5, the larger NAT particles $(r>3 \mu \mathrm{m})$ cannot be distinguished from STS. Therefore, we are unable to determine a positive identification of the presence of any NAT PSCs from these MIPAS observations.

An orbit transect, passing through the $\mathrm{HNO}_{3}$ depleted region on 25 May, is shown in detail in Fig. 7a-h. No large scale PSC occurrence is detected in association with the central low $\mathrm{HNO}_{3}$ region, which extends over $\sim 1200 \mathrm{~km}$ hori- zontally (equivalent to 8 MLS along-track measurement profiles) and $18-22 \mathrm{~km}$ vertically (Fig. 7f). Only patchy LIQ and MIX1 PSCs are detected (Fig. 7g), but the TTE correlates well with the depleted $\mathrm{HNO}_{3}$ (Fig. 7e, f), and much better than with the larger NAT existence region calculated from the local temperatures (Fig. 7b). For the region corresponding to TTE greater than 2-days, the $\mathrm{HNO}_{3}$ variation with temperature lies between the STS and NAT thermodynamic curves (Fig. 7d).

Vertical profiles along the same orbit transect discussed above are shown in Fig. 8a-d to highlight the depletion of $\mathrm{HNO}_{3}$ at $46-32 \mathrm{hPa}$ and the enhancement at $68 \mathrm{hPa}$. The CALIOP backscatter measurements are shown without any imposed PSC detection threshold or classification in Fig. 8a, $\mathrm{b}$ and without the spatial coherence constraint to show their considerable variation, particularly in the noisier perpendicular channel. At the lower pressures the total backscatter ratios become more evenly distributed about $R_{\mathrm{T}}=1.0$, reflecting the fall off in the sulfate background aerosol. Figure $8 \mathrm{e}-$ $\mathrm{h}$ shows the total and perpendicular backscatter, $\mathrm{HNO}_{3}$ and TTE selected for the pressure range $46-32 \mathrm{hPa}$. The total backscatter ratio and perpendicular backscatter both show increases at the lowest temperatures, but most of the data are below the detection threshold.

Selective nucleation of NAT within STS has been proposed by Larsen et al. (2004), who noted that previous lidar observations (Toon et al., 2000) also indicate that the liquid particles could evaporate as they are transported downwind of a synoptic-scale cold region, leaving the solid particles to persist in the warmer region. The 5-day back trajectories in Fig. 9 demonstrate the transport from the inner vortex, poleward of $82^{\circ} \mathrm{S}$, with GEOS-5 synoptic temperatures on the $475 \mathrm{~K}$ surface (and $500 \mathrm{~K}$, not shown) indicating prior constant exposure to temperatures $2-4 \mathrm{~K}$ below the NAT existence threshold temperature, and conditions capable of limited STS growth.

\subsubsection{Comparison with the STS-NAT model}

Consider again the lidar backscatter and depolarization expected for STS-NAT particle mixtures discussed in Sect. 4.2. For the case investigated in Figs. 6 and 7, the $\mathrm{HNO}_{3}$ will have been been reduced from the initial value over time by loss from sedimentation of large NAT particles. If we assume that loss by sedimentation of the largest particles removes 2 ppbv (the excess detected at the renitrified level) from the initial 14 ppbv total $\mathrm{HNO}_{3}$ abundance, then we can approximate better the total $\mathrm{HNO}_{3}$ at the height of the PSC at the measurement time as 12 ppbv. Therefore, since MLS measures 7 ppbv of gas-phase $\mathrm{HNO}_{3}$ at the PSC location, we can estimate the gas-phase fraction to be about $7 / 12$ or $58 \%$. Combined with the lidar observations, $R_{\mathrm{T}}<1.4$, and $\beta_{\perp}<$ $5 \times 10^{-6} \mathrm{~km}^{-1} \mathrm{sr}^{-1}$, Eq. (6) then indicates a large effective radius of $7 \mu \mathrm{m}$ and NAT number density $<1 \times 10^{-3} \mathrm{~cm}^{-3}$. The NAT number density lies at the lower end of the range 

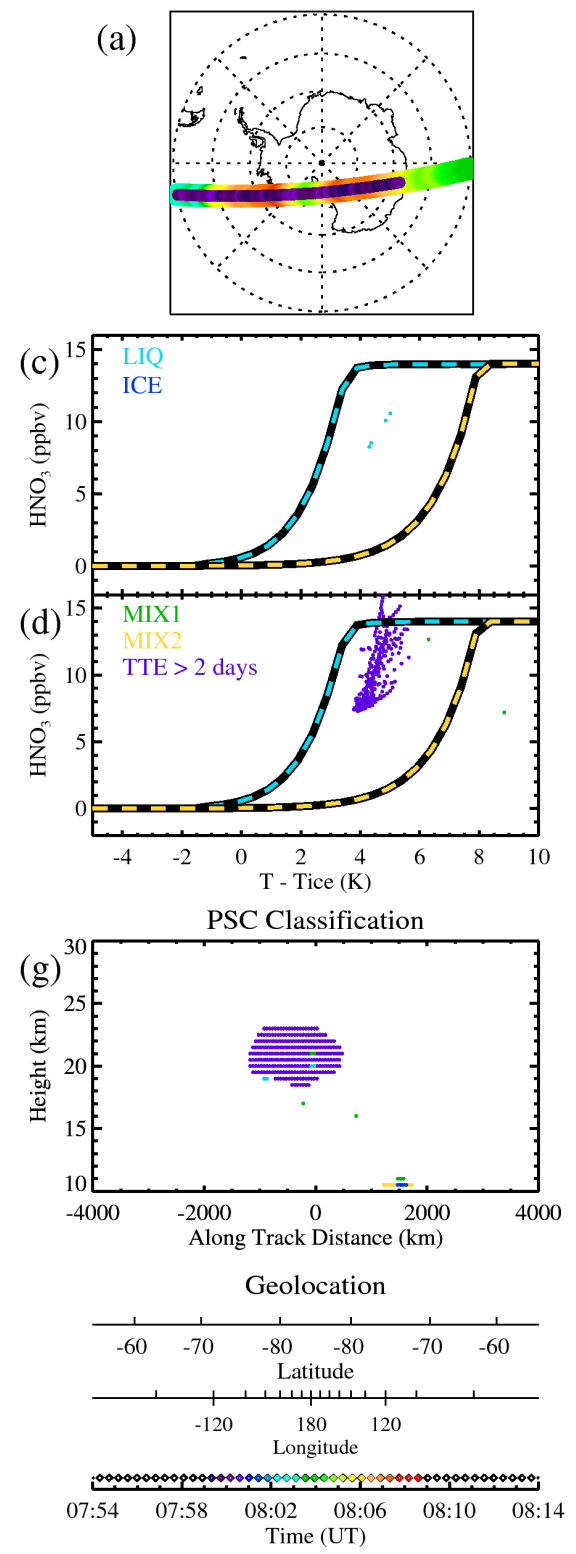

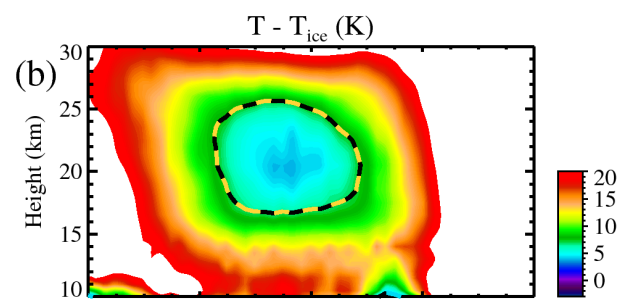

TTE (days)
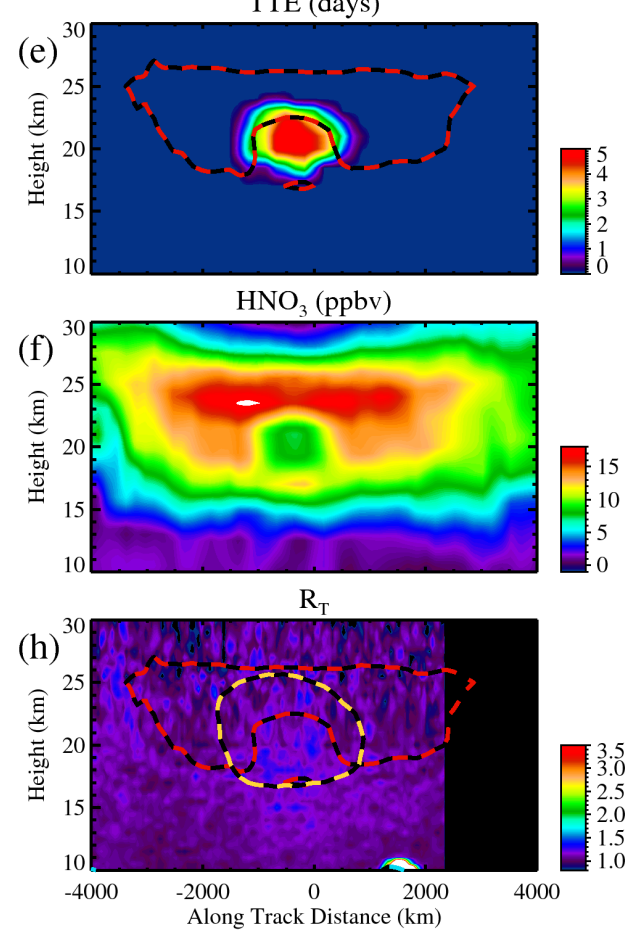

Geolocation

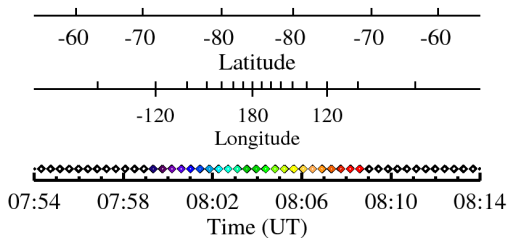

Fig. 7. (a) Geographic region sampled on an orbit track on 25 May 2008 through a denitrified region. The outer colored band represents the MLS $\mathrm{HNO}_{3}$ values at $22 \mathrm{~km}$ and the inner band represents the corresponding CALIOP backscatter ratio. The along-track distance is centred on the closest approach of the orbit track to the pole. (b) Temperature relative to the frost-point ( $T-T_{\text {ICE}}$ ). (c) Temperature distribution of MLS $\mathrm{HNO}_{3}$ corresponding to the CALIOP identified LIQ (cyan) and ICE (blue) PSC types (d) as (c) except for MIX1 (green) and MIX2 (yellow) PSC types and additionally for TTE $>2$ days (purple). (e) Temperature threshold exposure (TTE) in days. (f) MLS HNO 3 . (g) PSC type classification with additional background shading for TTE $>2$ days (purple). (h) CALIOP total backscatter ratios. Cyan-black and yellow-black dashed lines in (c, d) indicate the equilibrium curves for STS and NAT, respectively. Red-black dashed contours indicate the 12 ppbv $\mathrm{HNO}_{3}$ isopleth and yellow-black dashed contours in $(\mathbf{b}, \mathbf{h})$ indicate the $T_{\mathrm{NAT}}$ threshold temperature. All temperatures and temperature differences are given in Kelvin.

identified in the simulations by Jensen et al. (2002) as being most efficient for denitrification (see Sect. 2.6.2). Note that the assumed loss by sedimentation leaves 5 ppbv locked into the NAT particles, and it follows that we can only explain the low backscatter measurements by a combination of large NAT particle size and low number density. PSCs with smaller particle sizes and larger number densities, although allowed by the below-threshold backscatter measurements, are essentially ruled out by the $\mathrm{HNO}_{3}$ measurements because they would not contain a sufficient NAT volume to explain the observed gas-phase depletion of $\mathrm{HNO}_{3}$. 

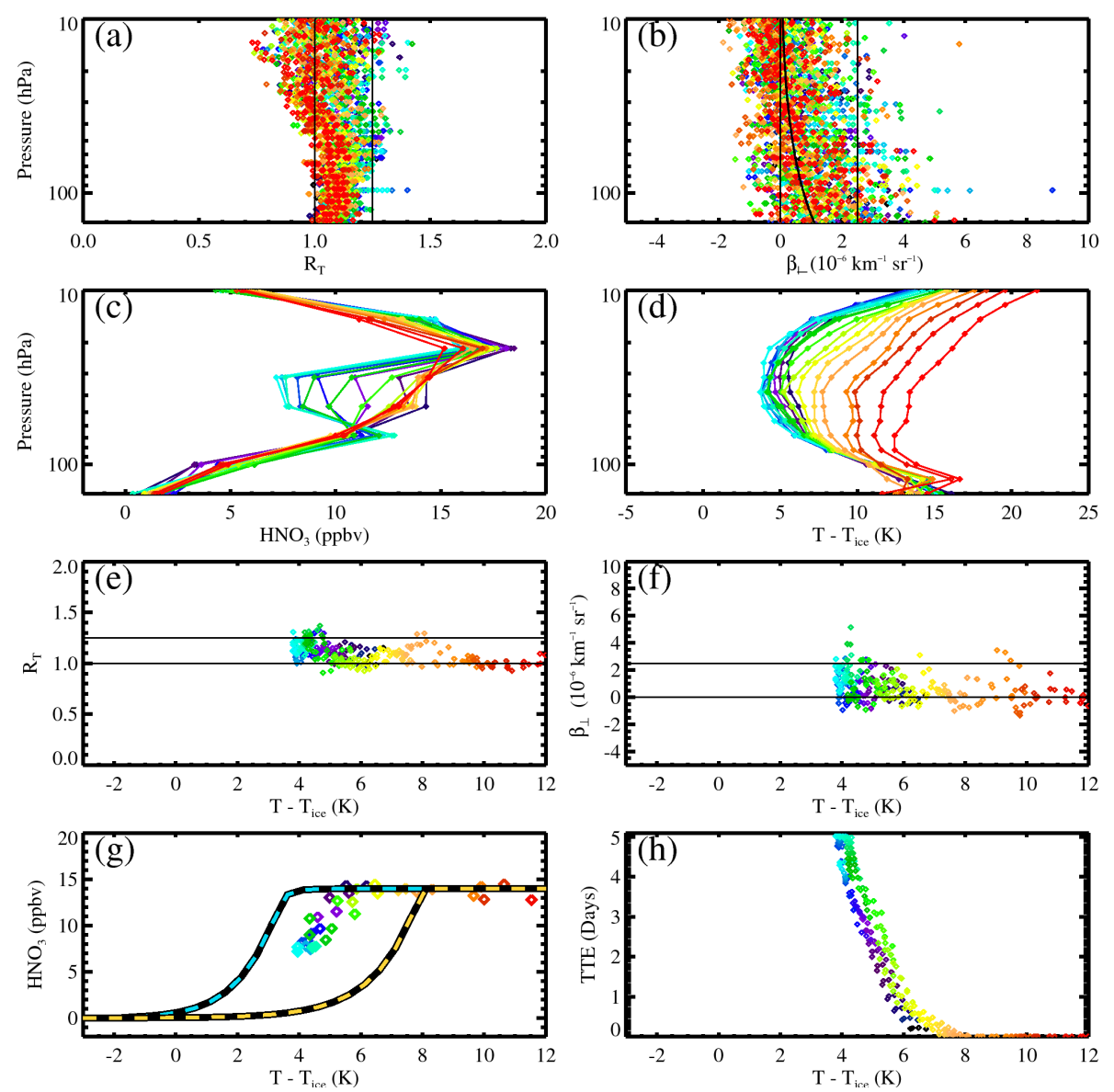

Fig. 8. Upper panels (a-d): vertical profiles of (a) total backscatter ratio, (b) perpendicular backscatter coefficient, (c) $\mathrm{MLS} \mathrm{HNO}_{3}$, (d) temperature relative to the frost-point $\left(T-T_{\mathrm{ICE}}\right)$. Lower panels $(\mathbf{e}-\mathbf{g})$ : scatter plots against temperature relative to the frost-point for quantities on the combined 46 and $32 \mathrm{hPa}$ pressure surfaces, where (e-g) are for the same quantities shown in (a-c). (h) Temperature threshold exposure (TTE) for $T-T_{\mathrm{ICE}}<5 \mathrm{~K}$ (in days). Data are color coded according to their location along the orbit track given in Fig. 7. CALIOP data are shown without an imposed PSC classification and MLS data are shown in $(\mathbf{c}, \mathbf{g})$ at their original retrieved resolution without interpolation. Thin solid black lines indicate backscatter ratios of 1.0 and 1.25 in (a, e) and perpendicular backscatter values of 0 and $2.5 \times 10^{-6} \mathrm{~km}^{-1} \mathrm{sr}^{-1}$ in $(\mathbf{b}, \mathbf{f})$, respectively. The curved line in (b) shows the calculated perpendicular backscatter arising from molecular scattering, which has been subtracted from the observed perpendicular backscatter. All temperatures and temperature differences are given in Kelvin.

\subsubsection{Summary of the early onset denitrification event}

- Rapid gas-phase $\mathrm{HNO}_{3}$ depletion in the central vortex region is accompanied by vertical redistribution, with renitrification occurring at lower heights.

- The depleted $\mathrm{HNO}_{3}$ region is correlated with exposure to temperatures several kelvin above the frost-point.

- The combination of CALIOP and MLS data indicates the presence of large NAT particles with a low number density.

- No positive NAT infrared spectral detections are found by MIPAS, but the observations are consistent with large-size NAT particles.
- No dehydration is observed by MLS during this episode, and typical values of $\mathrm{H}_{2} \mathrm{O}$ are $\sim 5$ ppmv.

Further denitrification events after this initial time period are more difficult to interpret and will require a more extensive modeling investigation, as they take place in a cooling atmosphere, where uptake in STS starts to prevail and the previously denitrified air is transported around the polar vortex.

\subsection{Development of NAT PSCs in the polar stratospheric belt}

\subsubsection{Mountain wave detection using AIRS radiances}

Figure 10 shows the development of the GEOS-5 temperatures at $40 \mathrm{hPa}$ over the Antarctic Peninsula region from 19 May-3 June 2008, and the detection of gravity wave 


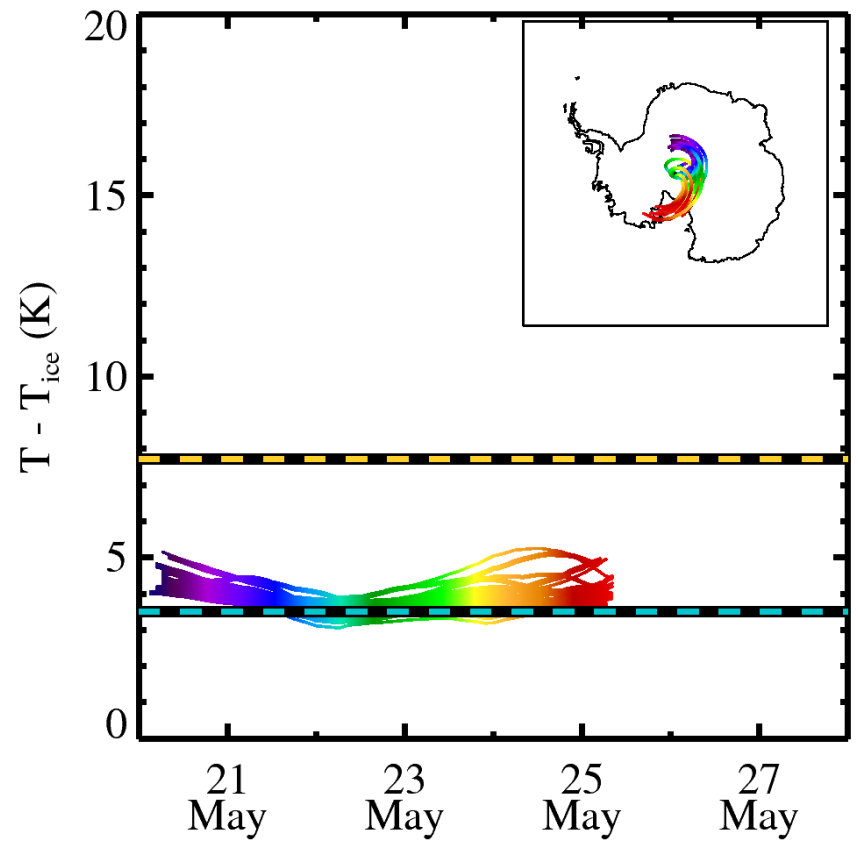

Fig. 9. Trajectory analysis of locations identified as depleted in $\mathrm{HNO}_{3}$ (less than $9 \mathrm{ppbv}$ ) on the MLS $46 \mathrm{hPa}$ pressure surface over the Ross Sea on 25 May 2008. Isentropic back trajectories are shown for $475 \mathrm{~K}(40 \mathrm{hPa})$ for a five day integration time. The inset geographical map shows that the likely origin overlies the inner vortex over the South Pole. Colors indicate the time (start (red) and end (purple)) along the trajectory. The corresponding GEOS5 synoptic-scale temperature analysis time histories show constant exposure to conditions $2-4 \mathrm{~K}$ below the NAT existence threshold temperature (yellow-black dashed line), with some air masses experiencing temperatures below the STS formation temperature (cyan-black dashed line).

activity near $40 \mathrm{hPa}$ by the AIRS instrument. The gravity wave activity at $80 \mathrm{hPa}$ (not shown) peaks on the same days as seen at $40 \mathrm{hPa}$, although in general there are more days with positive detections at $80 \mathrm{hPa}$. Moreover, some days with substantial activity at $80 \mathrm{hPa}$, particularly 23 May, show little or no activity at $40 \mathrm{hPa}$, which may be related to the AIRS vertical wavelength attenuation. There are two main periods of gravity wave activity detected at $40 \mathrm{hPa}$. The first period occurs during 20-22 May, when the background temperatures indicated by GEOS-5 are near or above the NAT existence temperature. This gravity wave event may be the cause of an isolated PSC (not shown) that appears at $20-22 \mathrm{~km}$ over the Weddell Sea on 23 May that is classified as LIQ with an overlaying MIX1 layer. The second episode of gravity wave activity occurs during a cooler period on 28-29 May, with synoptic temperatures up to $4 \mathrm{~K}$ lower than in the first period, and therefore the chances of mesoscale temperature fluctuations occurring below the ice frost point are increased. Additionally, individual AIRS scenes were examined to determine wave amplitudes and phases, and the second period was found to have larger brightness temperature oscillations. Gravity wave amplitudes of over $10 \mathrm{~K}$ against the synoptic temperature background would be required on 28-29 May in order to produce cooling of a few kelvin below the frost point, which is expected to lead to production of ice (Koop et al., 1998).

\subsubsection{Estimation of peak wave amplitudes}

In general, global analyses (Eckermann et al., 2006) are unable to resolve gravity waves accurately enough to provide reliable estimates of the actual wave amplitudes. However, the GEOS-5 temperatures do show oscillatory features on several days (e.g., 28 May) that appear to be correlated with the AIRS detections. Attenuation by the AIRS vertical response function also precludes a direct estimate of the temperature wave amplitudes; however, for the AIRS data in 2003, Eckermann et al. (2009) determined a theoretical vertical wavelength of $12 \mathrm{~km}$ from the wind speed and used the calculated wave-attenuation for the $40 \mathrm{hPa}$ detection to estimate a probable lower amplitude limit of about $10 \mathrm{~K}$. They argued further that, after taking into consideration the additional attenuation from data smoothing, the peak amplitudes could be as high as $15 \mathrm{~K}$. Similar considerations apply to the observations shown here and also indicate that local temperatures over the Antarctic Peninsula were sufficiently low to produce ice PSCs on 28-29 May.

\subsubsection{MIPAS observations}

On 28 May, in addition to the detection of activity by AIRS, a gravity wave feature is clearly seen in the GEOS5 temperature contours over the Palmer Peninsula (Fig. 10), and MIPAS first detected ice PSCs at altitudes of 20 $23 \mathrm{~km}$. The evolution of PSCs detected by MIPAS from 28 May-3 June 2008 and the MIPAS PSC color-ratio classification scheme (Höpfner et al., 2006b) are shown in Fig. 11 (no data were obtained on 31 May). The PSCs classified as NAT stand out quite markedly against the background of STS detections, appearing downstream of the Antarctic Peninsula, clustered at the most northerly latitudes and with maximum cloud opacities occurring at greater altitudes. At first sight the forward trajectories calculated from the MIPAS ice locations and times in Fig. 11d indicate only marginal evidence for ice seeding, since just two observations of the MIPAS NAT PSCs were detected at later related times, contained in a downstream trajectory "corridor" within one day of the initial ice detections. Most of the MIPAS NAT PSCs were detected several days later at apparently unrelated locations. However, the limitations of spatial and temporal sampling by a satellite instrument must be factored into the chances of detection of small-scale ice PSCs associated with their generation in the cold phases of gravity waves. 


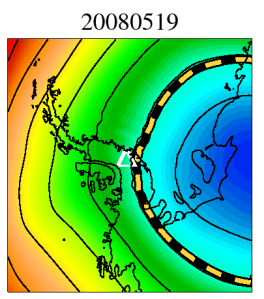

20080523

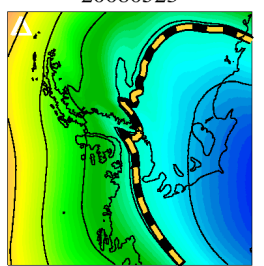

20080527

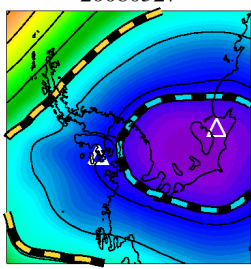

20080531

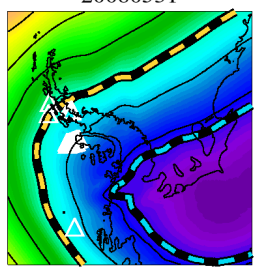

20080520

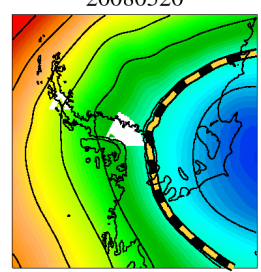

20080524

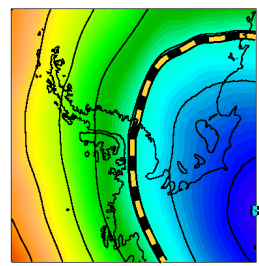

20080528

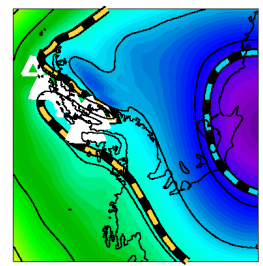

20080601

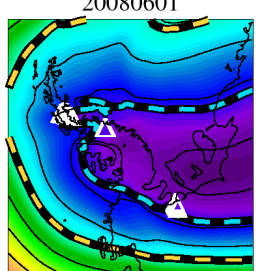

20080521

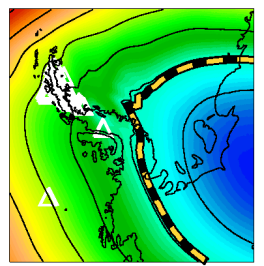

20080525

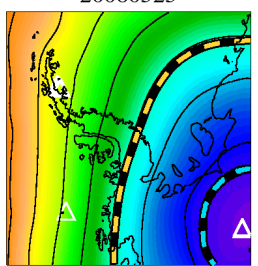

20080529

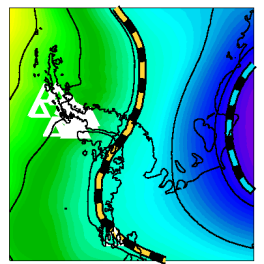

20080602

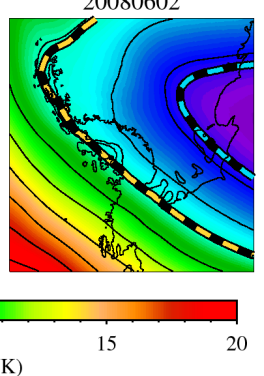

20080522

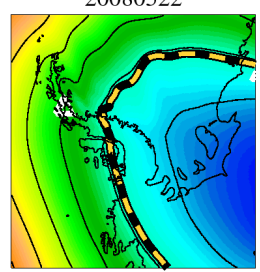

20080526

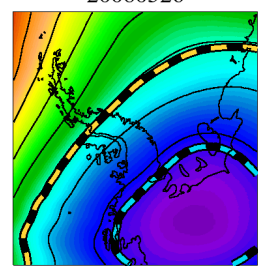

20080530

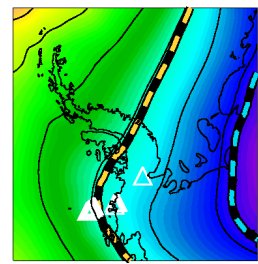

20080603

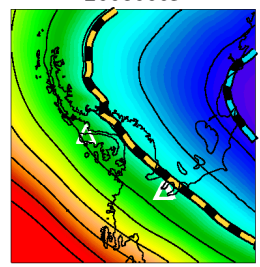

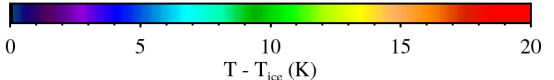

Fig. 10. AIRS detections of gravity waves (white triangles) at $40 \mathrm{hPa}$ from $19 \mathrm{May}-3$ June 2008 over the Antarctic Peninsula region. Colored shading shows the GEOS-5 temperatures relative to the the frost point, $T_{\mathrm{ICE}}$, in Kelvin, with red hues indicating higher temperatures than purple hues (solid black contours are at $2 \mathrm{~K}$ intervals). Highlighted contours show the existence threshold temperature of NAT (approximately $T_{\text {ICE }}+7.5 \mathrm{~K}$, yellow-black dashed) and formation temperature of STS ( $T_{\mathrm{ICE}}+3.5 \mathrm{~K}$, cyan-black dashed).

\subsubsection{CALIOP and MLS observations}

The evolution of PSCs detected by CALIOP at $22 \mathrm{~km}$ from 28 May-2 June 2008 is shown in Fig. 12, along with the contours of the NAT existence temperature and the STS formation temperature calculated from the GEOS-5 temperatures and $\mathrm{MLS} \mathrm{H}_{2} \mathrm{O}$ and $\mathrm{HNO}_{3}$. The corresponding $\mathrm{HNO}_{3}$ values observed by MLS are shown in Fig. 13.

The first CALIOP PSCs detected in the polar belt during the 28-29 May gravity wave activity are located just downstream of the Peninsula on 28 May (see orbit tracks 10 and 11 of Fig. 12a). Subsequently, MIX2 PSCs are observed in the polar belt in the altitude range $18-26 \mathrm{~km}$, with a typical vertical extent of $4 \mathrm{~km}$ and horizontal extents of up to $1000 \mathrm{~km}$, throughout the next six days of CALIOP observations (no data were obtained during 4-9 June). Although missing the earlier MIPAS detections of ice on 28 May, CALIOP de- tected PSCs with large backscatter and depolarization that were classified as ice types from 30 May-1 June, and that were located in regions over the Antarctic Peninsula and the Ellsworth Mountains.

An example CALIPSO orbit transect through the central PSC region close to the pole and through the PSC region in the polar stratospheric belt is shown in Fig. 14a-h for 2 June. Three distinct PSC-containing areas are detected at altitudes $17-22 \mathrm{~km}$ in the central denitrified region (Fig. 14g), two composed mainly of the LIQ type and one composed mainly of MIX1 with a much smaller area of MIX2 and LIQ. At lower altitudes $14-17 \mathrm{~km}$, below the central region, MIX1 PSCs are detected extensively in a thick layer covering an along-track distance of $800 \mathrm{~km}$. There is evidence of renitrification at $15 \mathrm{~km}$ near along-track coordinate $-500 \mathrm{~km}$ (Fig. 14f) based on an increase in $\mathrm{HNO}_{3}$ above the maximum values seen at the same height prior to the onset of 
(a)

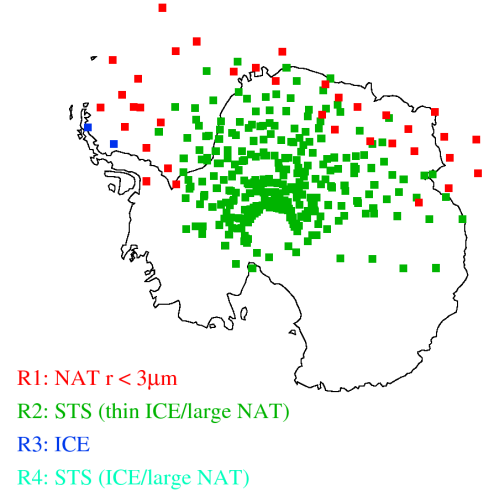

(c)

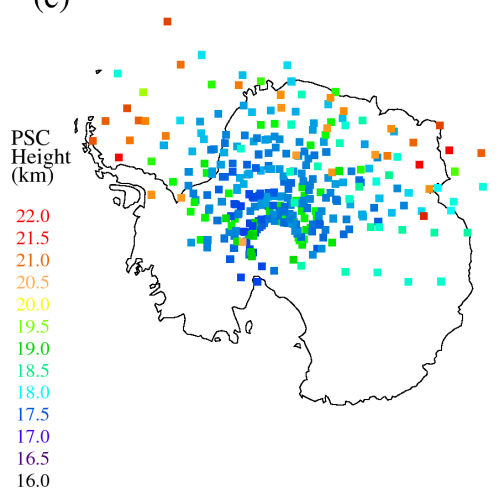

(b)

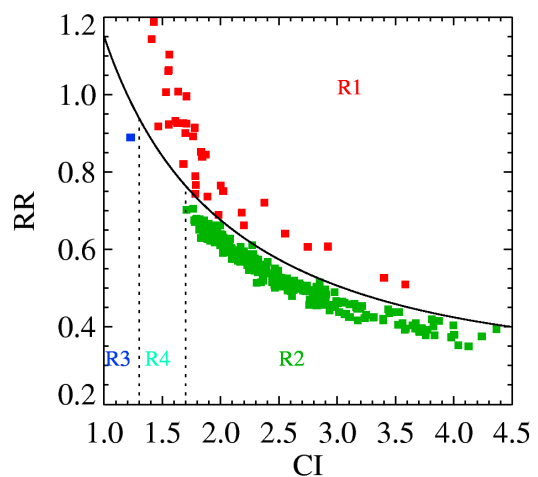

(d)

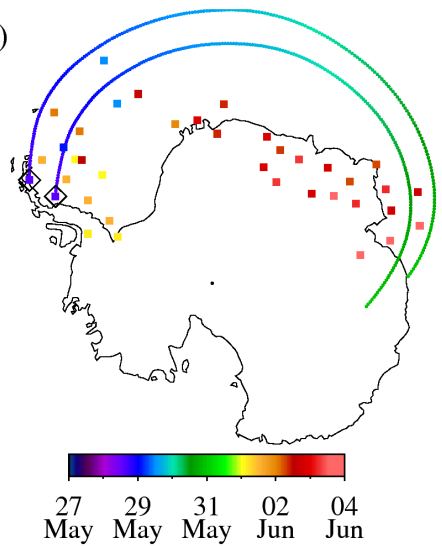

Fig. 11. Geographic distribution and spectral color-ratios of PSCs detected by MIPAS from 28 May-3 June 2008. (a) MIPAS PSC type classification $(\mathrm{CI}<4.5$, for all types $\mathrm{R} 1-4)$ at the altitudes corresponding to the maximum cloud opacity (minimum $\mathrm{CI}$ ) detected above the tropopause. (b) The distribution of color-ratios corresponding to (a). The region R1 contains NAT clouds (red square symbols) with mean particle radii $<3 \mu \mathrm{m}$ and well-defined NAT spectral signatures. R1 is separated from the other regions (R2-4) by a functional form (solid black curve) given by Höpfner et al. (2006b). Dashed lines show the regions most likely to contain ICE (R3 (blue), CI < 1.3) and STS (R4 (cyan), $1.7<\mathrm{CI}<1.3$ ). For the data in regions R2 (green) and R4, MIPAS is unable to distinguish between STS or large NAT with mean particle radii $>3 \mu \mathrm{m}$. (c) PSC altitudes corresponding to maximum cloud opacity for the data in (a) and color-coded according to the scale at the left. (d) MIPAS PSC type classification for solid types only (R1 and R3). Forward trajectories on the $500 \mathrm{~K}$ potential temperature surface were launched from the locations shown by the diamond symbols at the times when PSCs classified as ice types were detected. The color scale in (d) indicates the PSC observation times and the time along the trajectories, such that trajectories passing near or intersecting with observations at near coincident times have similar colors.

denitrification, which we assume occurs via the sedimentation and evaporations of NAT PSCs. Observations of MLS $\mathrm{O}_{3}$ (not shown) remain fairly constant at the same height over the same period and indicate that this is not the result of diabatic descent. The renitrified area extends below the height of the NAT existence temperature contour in Fig. 14h, indicating the likelihood of eventual evaporation of NAT particles which descend into this warmer region.

The $\mathrm{HNO}_{3}$ uptake by the liquid particles and many of the MIX1 class follows the STS equilibrium curve (Fig. 14c, d), as does the temperature variation of the $\mathrm{HNO}_{3}$ distribution in the region corresponding to TTE greater than 2 days but excluding the detected PSCs (Fig. 14d, e). The large-scale PSC in the polar stratospheric belt is at an altitude of 19$24 \mathrm{~km}$, mainly composed of the MIX2 type with some MIX1 detected at the cloud edges and below. The TTE associated with the MIX2 PSC is less than 2 days. The $\mathrm{HNO}_{3}$ uptake associated with the MIX2 PSC (Fig. 14d) shows a large variation and is skewed towards the NAT equilibrium curve. There is little residual gas-phase $\mathrm{HNO}_{3}$ in the center of the MIX2 PSC region (Fig. 14f), and below the MIX2 PSC we do not see an extensive MIX1 region.

\subsubsection{Characteristics of the polar belt NAT PSCs}

Vertical profiles along the CALIPSO orbit transect are shown in Fig. 15a-d that highlight the highly depolarizing nature and large $\mathrm{HNO}_{3}$ uptake by the MIX2 PSCs at temperatures $4-8 \mathrm{~K}$ above the frost point. Figure $15 \mathrm{e}-\mathrm{h}$ shows the total and perpendicular backscatter, $\mathrm{HNO}_{3}$ and TTE selected for the pressure range 46-32 $\mathrm{hPa}$. Examination of the 

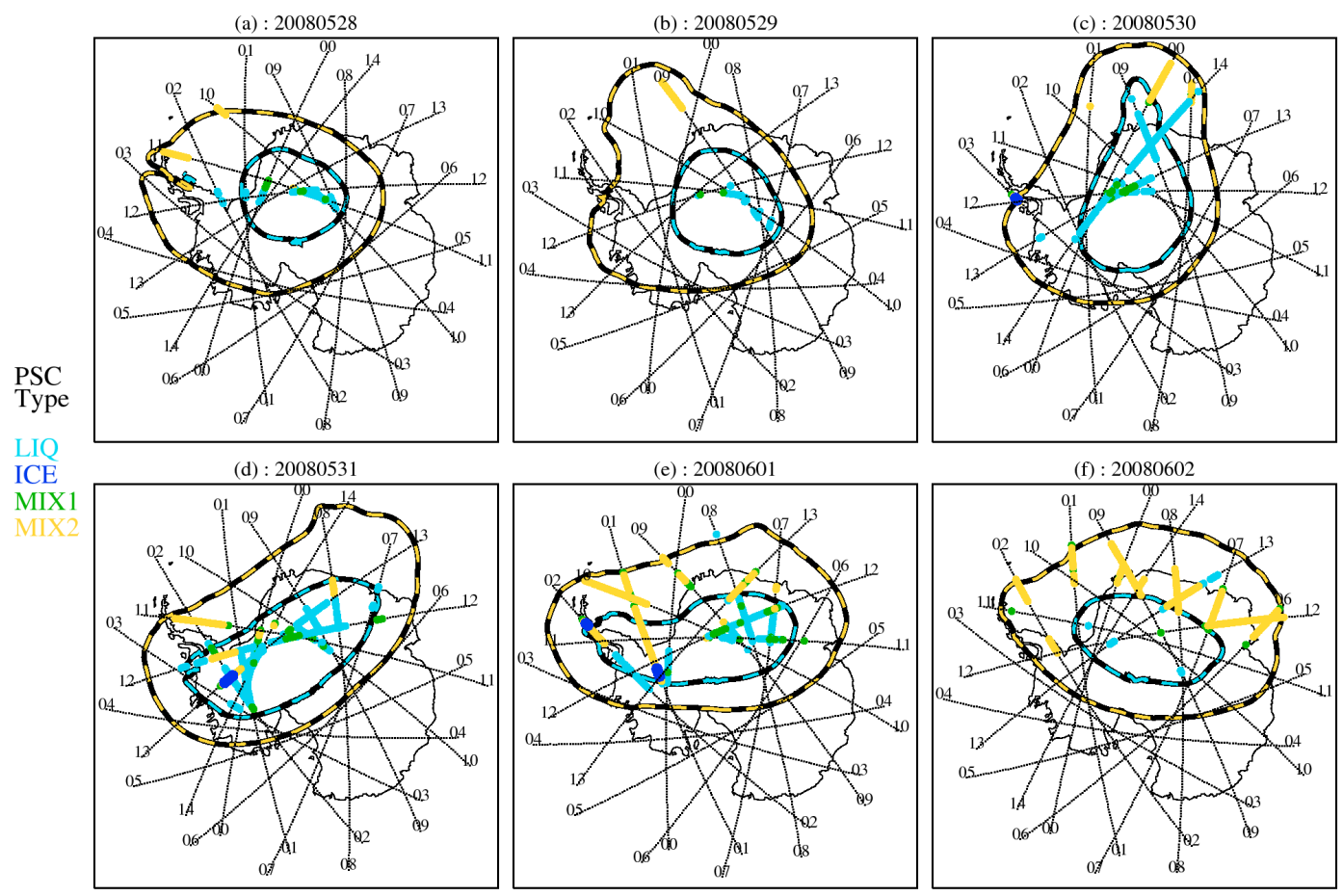

Fig. 12. Geographic distribution of the CALIOP PSC types detected at an altitude of $22 \mathrm{~km}$ from 28 May-2 June 2008, (a)-(f). Highlighted contours are the NAT existence threshold temperature (approximately $T_{\mathrm{ICE}}+7.5 \mathrm{~K}$, yellow-black dashed) and STS formation temperature $\left(T_{\mathrm{ICE}}+3.5 \mathrm{~K}\right.$, cyan-black dashed) calculated from the GEOS-5 temperatures and MLS $\mathrm{HNO}_{3}$ and $\mathrm{H}_{2} \mathrm{O}$ at $32 \mathrm{hPa}$. CALIOP detected ICE from 30 May-1 June over the Antarctic Peninsula and the Ellsworth Mountains. Black dots are observations without PSC detections.
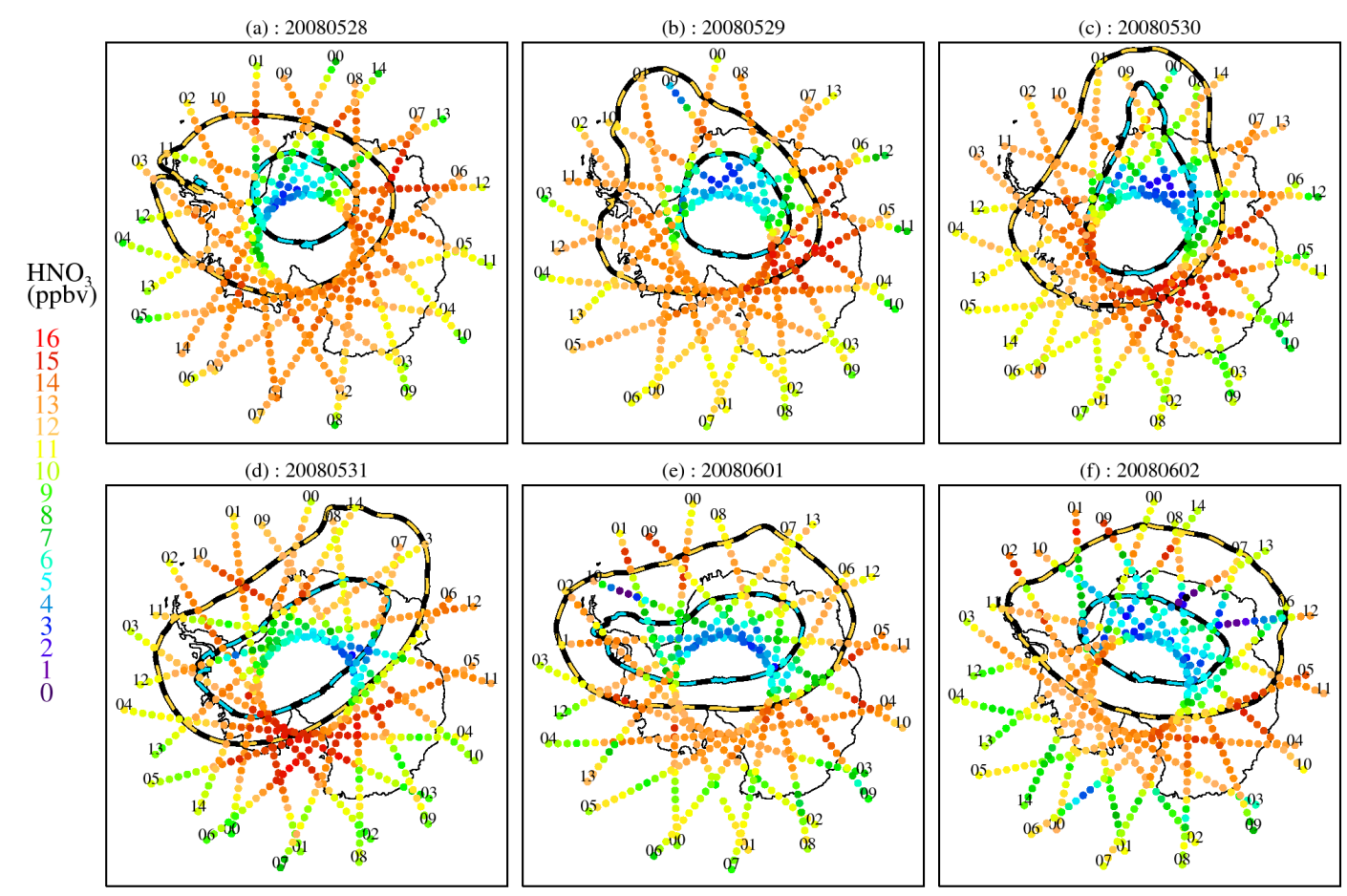

Fig. 13. As Fig. 12, except for $\mathrm{MLS} \mathrm{HNO}_{3}$ at $32 \mathrm{hPa}$. 


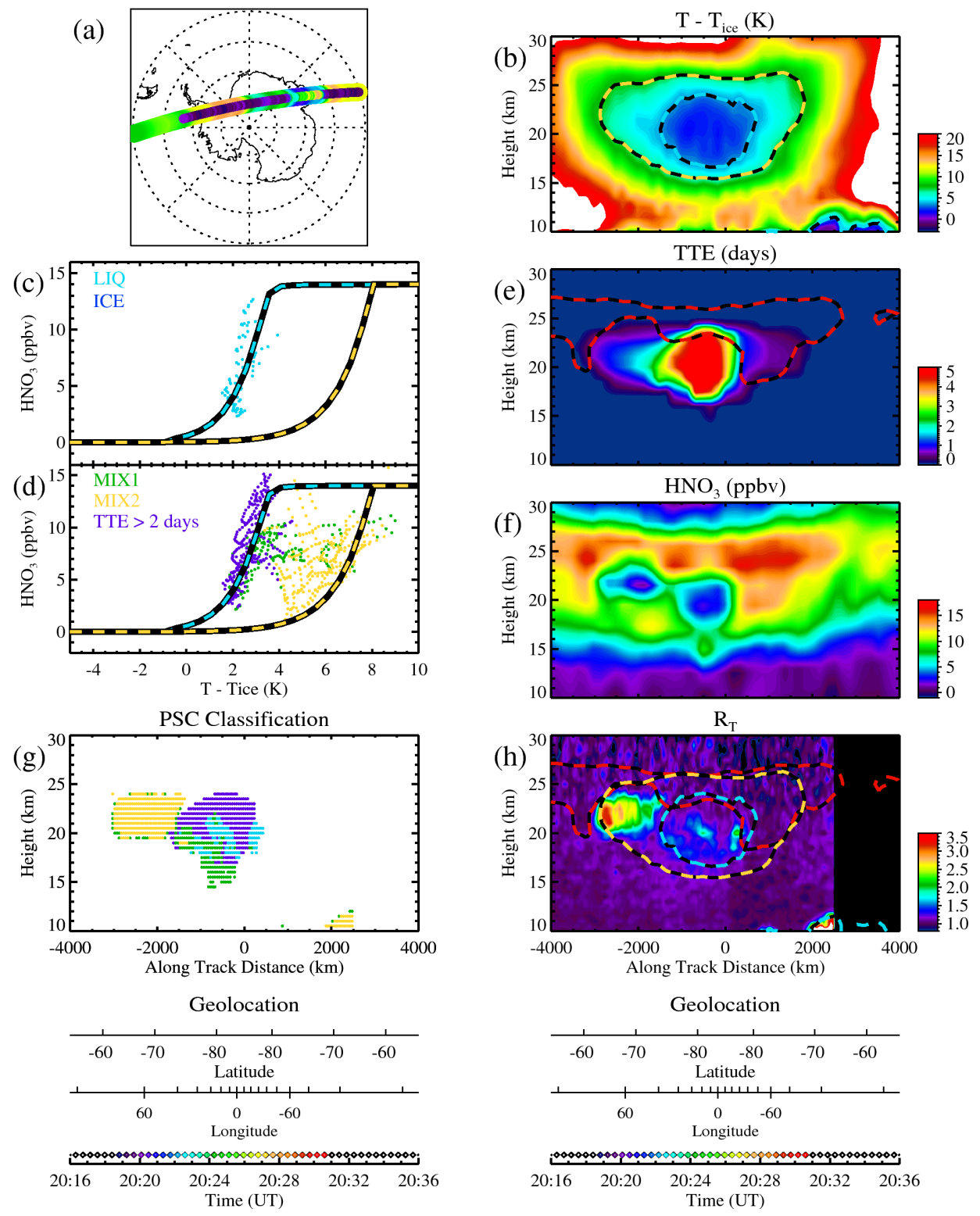

Fig. 14. As Fig. 7, but for orbit track 12 on 2 June 2008 (see Figs. 12f and 13f).

total backscatter and perpendicular backscatter show both to be high for this NAT cloud $\left(R_{\mathrm{T}} \sim 2.5-3.5, \beta_{\perp} \sim 35-\right.$ $55 \times 10^{-6} \mathrm{~cm}^{-3}$ ), compared to typical Type Ia NAT PSCs, which indicates a relatively small NAT particle size distribution and high NAT number density. We estimate that $r_{\mathrm{e}} \sim 0.5-1 \mu \mathrm{m}$ and $N>0.2 \mathrm{~cm}^{-3}$ from Eq. (6) (see also Fig. 4a, c, e).

Correspondingly, we associate this MIX2 PSC with the Type Ia enhanced classification described by Tsias et al. (1999) and the wave-induced PSCs detected by Hu et al. (2002). Such PSCs are characterized by formation by a mountain-wave mechanism with a composition closer to NAT thermodynamic equilibrium than the more prevalent
Type Ia clouds associated with synoptic-scale freezing. PSCs with similar characteristics were discussed by Toon et al. (2000), who also noted that smaller NAT particle sizes tend to form following a recent cooling, within a day or so, to temperatures below the NAT existence threshold. They contrasted this formation scenario with that commonly observed for the larger NAT particle sizes, which tend to form only following exposure for a few days to temperatures below the NAT threshold. Many of the MIX2 PSCs observed during the period from 28 May-3 June in the polar belt have similar characteristics. Additionally, some MIPAS PSC classifications in Fig. 11 show high values for the NAT spectral parameter, RR $>1.0$, which indicates the presence of NAT 

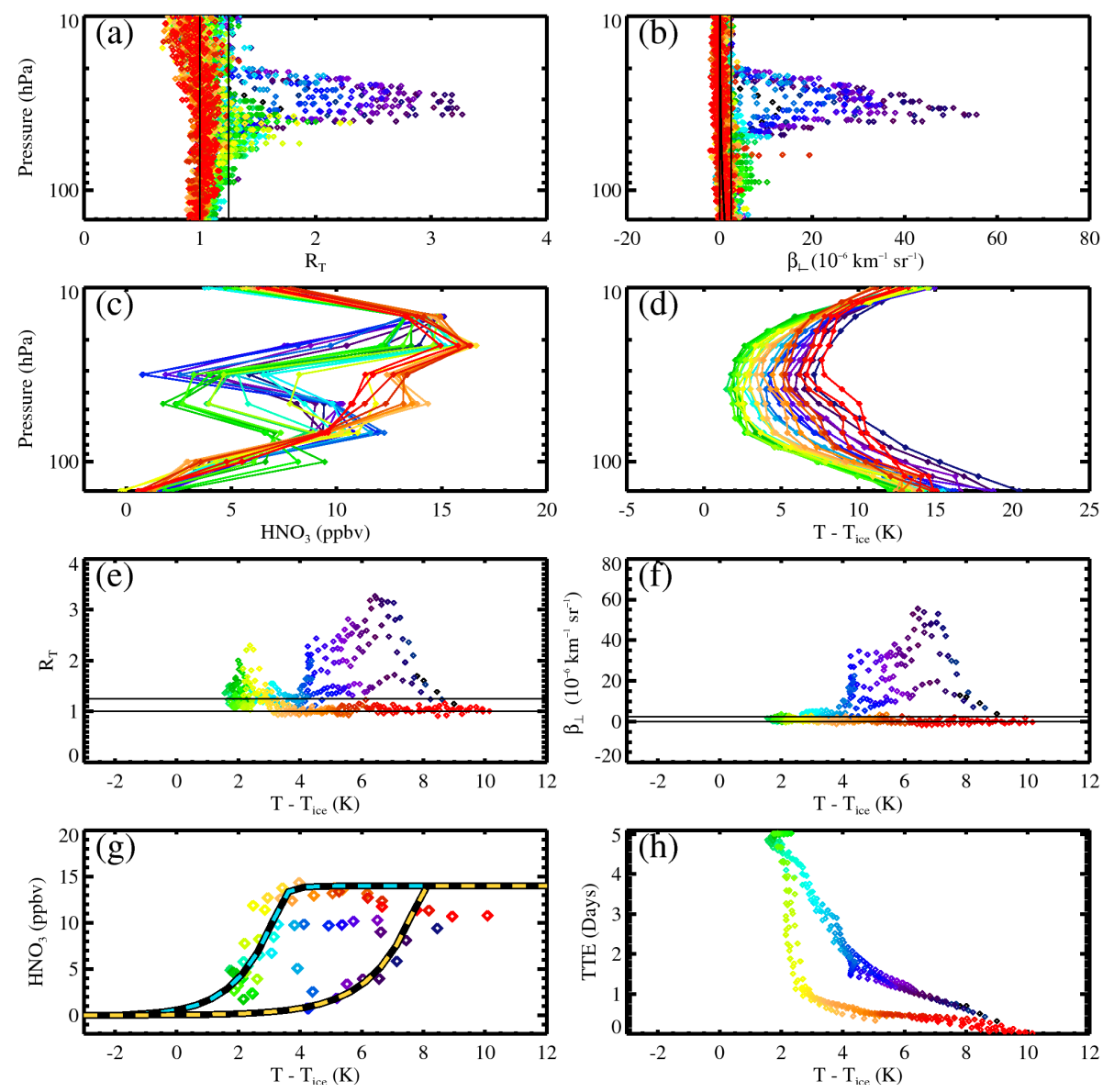

Fig. 15. As Fig. 8, but for orbit track 12 on 2 June 2008 (see Figs. 12f and 13f). Note scale changes in (a, b, e, f) from Fig. 8.

with mean particle radii $<1 \mu \mathrm{m}$ (Höpfner et al., 2006b). These observations suggest a nucleation process at play that activates a substantial fraction of the available nuclei. Lacking a sufficiently $\mathrm{HNO}_{3}$ rich environment because of the uptake by a large number of small NAT particles, further growth may be inhibited in these MIX2 clouds, thereby preventing rapid sedimentation (see Sect. 2.6.2).

Figure 16 shows composite orbit plots for the CALIOP PSC classifications and $\mathrm{HNO}_{3}$ distribution corresponding to locations of the PSCs detected for the period 28 May-3 June at $22 \mathrm{~km}$. An additional CALIOP PSC classification has been generated to denote the sub-class of NAT-enhanced PSCs (NATE) identified by Tsias et al. (1999). Our definition corresponds to depolarization, $\delta_{\mathrm{p}}>0.2$ (Adriani et al., 2004) and backscatter ratio, $5>R_{\mathrm{T}}>1$.67. Pitts et al. (2011) described a similar extension to their original PSC classification scheme. Most of the MIX2 PSCs in the polar belt in Fig. 16 are seen to fall into the NATE category. The depolarization vs normalized backscatter of all the CALIOP PSCs detected from 28 May-3 June 2008 is shown in Fig. 17. As noted previously in Sect. 4.1, a constraint was added to ensure that only PSCs with significant perpendicular backscatter are ac- cepted into the MIX1 class. This results in a broader range of the noisy positive depolarization values being placed in the LIQ classification at lower total backscatter.

\subsubsection{Evidence for ice seeding}

In order to investigate further the potential for ice seeding of NAT clouds and their development downstream from the region of initial ice formation, forward trajectories were calculated starting from the locations and times that the CALIOP PSCs classified as ice were detected over the Palmer Peninsula on 30 May and 1 June and the Ellsworth Mountains on 31 May and 1 June. These trajectories are found to enclose the region downstream where the MIX2 PSCs are detected from 31 May-3 June. On 2 June the northward locations of the MIX2 PSCs are particularly well bounded by these forward trajectories (see Fig. 16) compared to the much wider demarcation area of the NAT existence temperature (see Fig. 12f).

Pitts et al. (2011) determined that wave-ice clouds can be identified through the presence of very large backscatter ratios $\left(R_{\mathrm{T}}>50\right)$, which also show lidar color ratios in the range $0.25-0.35$, indicating high ice particle number densities and 
(a)

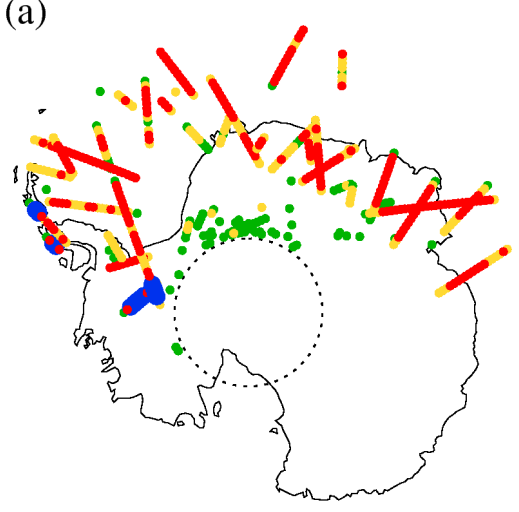

MIX1 MIX2 NATE ICE

(c)

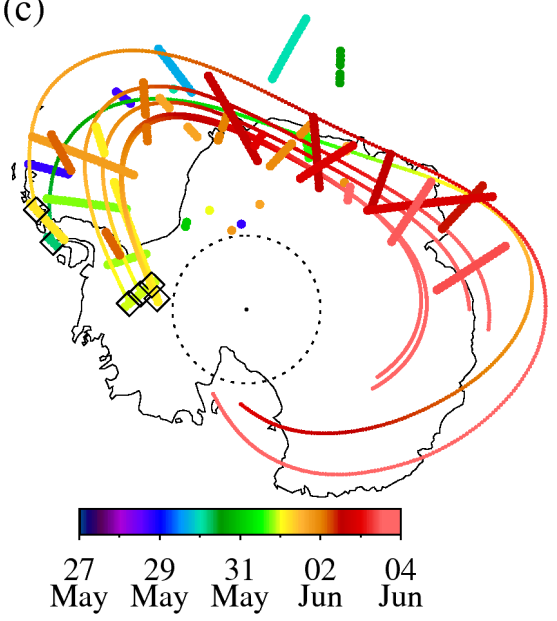

(b)

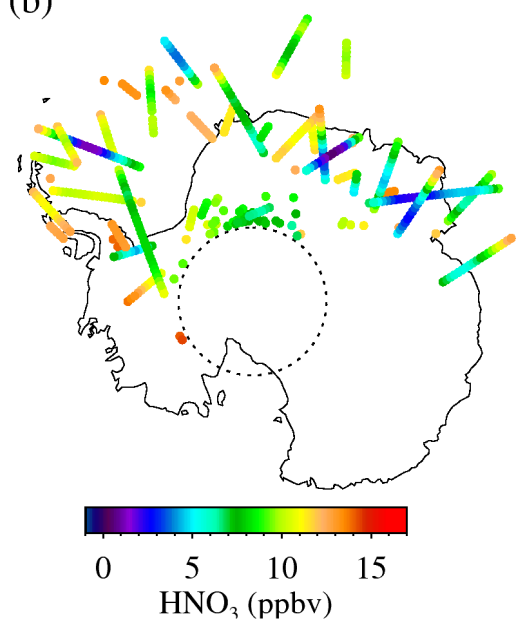

(d)

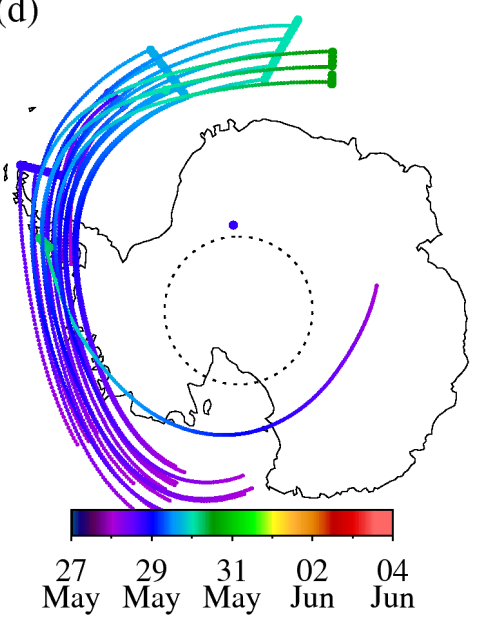

Fig. 16. Geographic distribution of CALIOP and MLS data at an altitude of $22 \mathrm{~km}$ for the period 28 May-3 June 2008. A dashed latitude circle at $82^{\circ} \mathrm{S}$ indicates the limit of the CALIOP and MLS observations. (a) CALIOP PSC classification for solid types only (MIX1 (green), MIX2 (yellow), ICE (blue)) and including the enhanced-NAT sub-category (NATE (red)). (b) MLS $\mathrm{HNO}_{3}$ corresponding to the PSC locations in (a), typical unperturbed values would be $\sim 12$ ppbv. (c) Forward trajectories on the $500 \mathrm{~K}$ potential temperature surface were launched from the locations shown by the diamond symbols at the times when PSCs classified as ICE types by CALIOP were detected. (d) Backward trajectories on the $500 \mathrm{~K}$ potential temperature surface were launched from a representative sample of points along the CALIOP orbit tracks at the times when MIX2 PSCs were detected during 28-30 May. The color scales in (c,d) indicate both the PSC observation times and the time along the trajectory tracks, such that trajectories passing near or intersecting with observation tracks at near coincident times have similar colors. Only MIX2 PSCs (including NATE) are shown for clarity in (c, d).

small particle radii of $1-1.5 \mu \mathrm{m}$. The horizontal averaging used in our investigations leads to a reduction in the apparent peak backscatter for PSCs with a horizontal extent much smaller than $50 \mathrm{~km}$. Analysis of the higher resolution $5 \mathrm{~km}$ data (not shown) confirms that the ICE type PSCs with high backscatter detected using the $50-\mathrm{km}$ gridding during this time are further classified as wave-ice.

As already noted above, most of the MIPAS NAT detections are not trajectory time-correlated with the initial MIPAS ice detection on 28 May. However, as we see from the geographic distributions in Figs. 11d and 16c, there is a good overall correspondence in the times and locations of the MIPAS NAT and CALIOP MIX2 detections and with the "cor- ridor" formed by the trajectories from the CALIOP ice detections. Back trajectories were calculated from the locations and times of observations of the MIX2 clouds on 28-30 May, and these also indicate passage over the Palmer Peninsula following the start of the gravity wave activity and the first ice detection by MIPAS on 28 May.

\subsubsection{Temperature histories}

Several of the MIX2 PSC detections in the polar belt that are located well away from the Antarctic Peninsula show temperature histories such as those in Fig. 18, where the air parcels undergo a thermal cycling of $5 \mathrm{~K}$ peak to peak over a period 


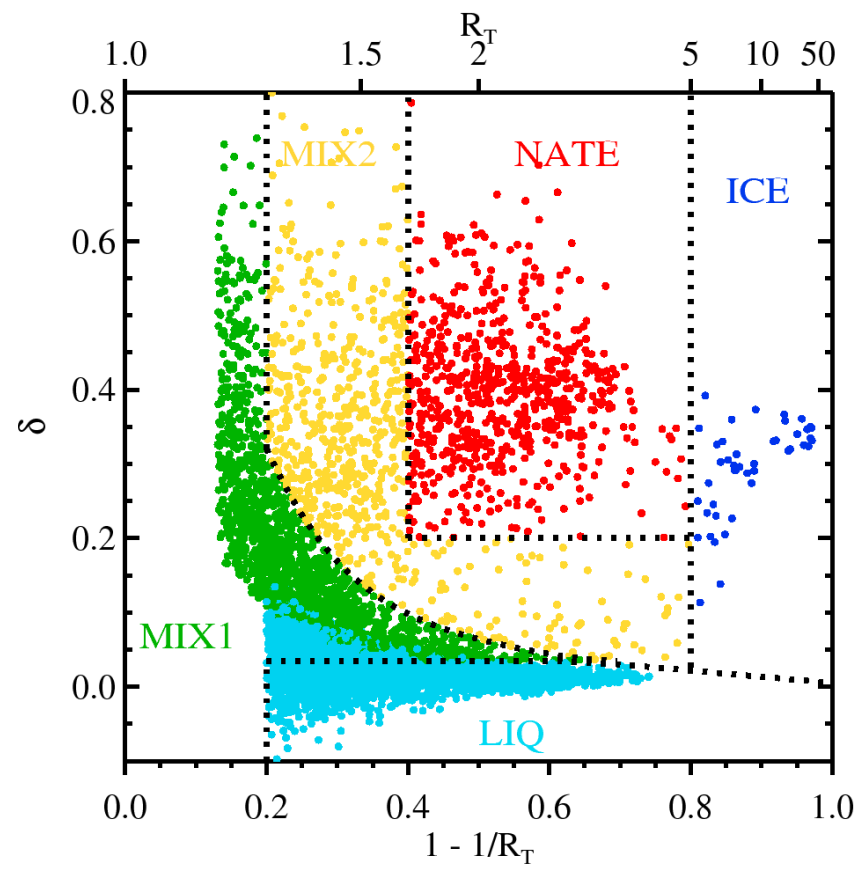

Fig. 17. Scatter plot of the depolarization vs. normalized backscatter for the period 28 May-3 June 2008, showing the PSC types identified by CALIOP (MIX1 (green), MIX2 (yellow), LIQ (cyan), ICE (blue)) and including the enhanced-NAT sub-category (NATE (red)) at an altitude of $20-22 \mathrm{~km}$.

of about two days following the passage over the Peninsula. The lowest synoptic temperatures are only $2-3 \mathrm{~K}$ above the frost point and so readily decreased below $T_{\mathrm{ICE}}$ by mesoscale fluctuations. Also of note is that although the thermal cycling sometimes causes temperatures to exceed the NAT existence threshold, the MIX2 PSC either survived melting or reformed further downstream. Other examples of MIX2 PSCs detected in locations closer to the Antarctic Peninsula have experienced little exposure to synoptic-scale temperatures less than $5 \mathrm{~K}$ above the frost point. The back trajectories for the example in Fig. 19 indicate visible temperature fluctuations during the passage over the Antarctic Peninsula, less than one day earlier than the MIX2 PSC observation by CALIOP, which corresponds to the time of increased wave activity detected by AIRS. As discussed in Sect. 4.5.2, the actual temperature wave amplitudes are probably much larger $(>10 \mathrm{~K})$.

\subsubsection{Summary of the NAT polar belt outbreak}

- Mountain waves were detected over the Palmer Peninsula by AIRS with temperature fluctuations of over $10 \mathrm{~K}$.

- MIPAS detected ice coincident with the mountain wave activity, and CALIOP identified wave-ice clouds over the Palmer Peninsula and Ellsworth Mountains.

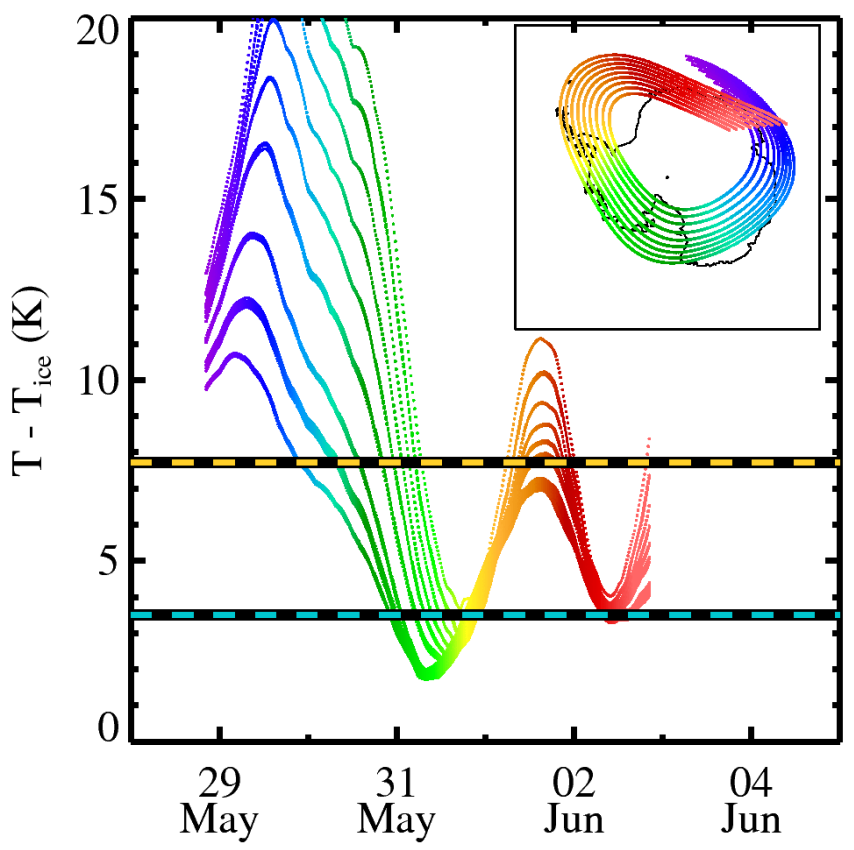

Fig. 18. Back trajectories calculated for the $500 \mathrm{~K}$ isentropic level for a MIX2 PSC detected along orbit track 12 on 2 June 2008 (see Figs. $12 \mathrm{f}$ and $13 \mathrm{f}$ ) in the polar stratospheric belt showing the passage over the Antarctic Peninsula (inset geographical map) and the synoptic-scale temperature history. Colors indicate the time (start (red) and end (purple)) along the trajectory. The yellow-black and cyan-black dashed lines indicate the NAT existence temperature and STS formation temperatures, respectively.

- MIPAS identified NAT clouds with a strong infrared spectral signature, and CALIOP classified these as NATenhanced types.

- MIPAS, CALIOP and MLS observations indicate the presence of small NAT particles with a large number density.

- Trajectory analyses are consistent with the development of the NAT clouds following ice seeding.

CALIOP operations were suspended during 4-9 June, and after data collection resumed on 10 June MIX1/MIX2 PSCs occupied a longitude region extending over the western half of the continent. The vortex temperatures were considerably lower than during the previous episode, and although they were below the ice formation threshold in the central vortex, LIQ was the prevalent PSC class and ICE was not detected until 14 June. MIX2 PSCs continued to be detected sporadically in the polar belt, and denitrification of the vortex became more extensive. A second episode of formation of MIX2 PSCs in the polar belt is clearly evident during 21-23 June in the CALIOP data. 


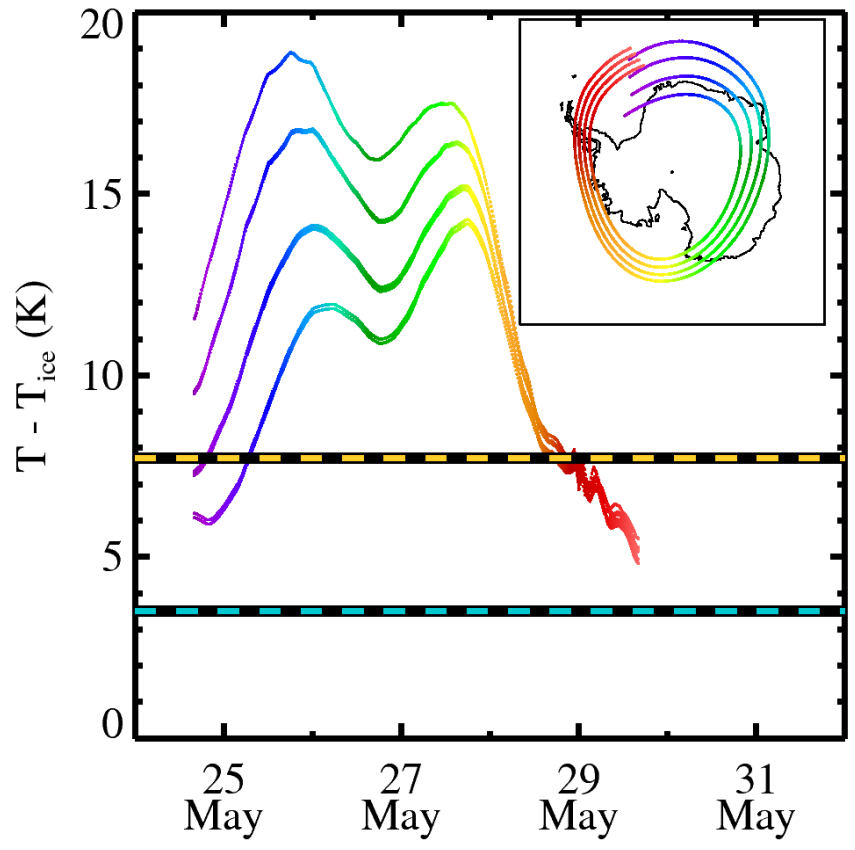

Fig. 19. As Fig. 18 but for a MIX2 PSC detected along orbit track 9 on 29 May 2008 (see Figs. 12b and 13b).

\section{Conclusions}

Combined observations from the A-train instruments CALIOP and MLS were used to investigate the development of PSCs through lidar backscatter signals and to determine their effects on the gas-phase nitric acid distribution during the early Antarctic winter in May-June 2008. Our results illustrate the benefits of combining these measurements, which provide better temporal and spatial coincidences for PSC and gas-phase measurements than previously achieved from instruments operating on different satellite platforms. Observational evidence of gravity-wave activity was provided by Aqua AIRS radiances, and infrared spectroscopic detection of NAT-containing PSCs was obtained from EnviSat MIPAS. GEOS-5 analyses were used to derive Lagrangian trajectories and to determine temperaturetime histories of air parcels.

We used CALIOP backscatter and depolarization measurements to classify PSCs and the MLS measurements to determine the corresponding gas-phase $\mathrm{HNO}_{3}$ as a function of temperature. The observed uptake of nitric acid in liquid and solid PSCs was compared to model calculations assuming equilibrium thermodynamics. For liquid PSCs the uptake of $\mathrm{HNO}_{3}$ follows the theoretical STS equilibrium curve (Carlsaw et al., 1994), but at temperatures about $1 \mathrm{~K}$ lower as determined from GEOS-5. The depletion of the gas-phase $\mathrm{HNO}_{3}$ by solid PSCs (classed as MIX1/MIX2) occurs over a wider range of temperatures $(+2$ to $-7 \mathrm{~K})$ distributed about the NAT equilibrium curve.
Optical properties of external mixtures of STS and NAT PSCs, calculated using Mie theory and $T$-matrix methods, were used to simulate the temperature dependence of the expected backscatter signals and $\mathrm{HNO}_{3}$ gas-phase uptake for a range of NAT particle size distributions and number densities. Large NAT particle PSCs at low concentrations can sequester a significant fraction of $\mathrm{HNO}_{3}$ from the atmosphere, causing gas-phase depletions that can be detected by MLS, but the PSCs may not yield detectable increases above the CALIOP lidar threshold.

The first appearance of denitrification in the Antarctic during 2008 occurs close to the South Pole prior to the formation of ice PSCs. Rapid gas-phase $\mathrm{HNO}_{3}$ depletion is first seen from 23-25 May 2008, consisting of a decrease from $14 \mathrm{ppbv}$ to $7 \mathrm{ppbv}$ on the $46-32 \mathrm{hPa}$ pressure levels and accompanied by a $2-3$ ppbv increase by renitrification at the $68 \mathrm{hPa}$ pressure level. It appears to be related to slow synoptic-scale cooling in the inner vortex, which led to the formation and growth of low number density $\left(<1 \times 10^{-3} \mathrm{~cm}^{-3}\right)$ large-size NAT particles (effective radii $>5-7 \mu \mathrm{m}$ ). Temperature-time histories of air parcels were used to quantify the duration of exposure to low temperatures and to demonstrate that the depleted $\mathrm{HNO}_{3}$ region is more clearly correlated with exposure to temperatures less than $5 \mathrm{~K}$ above the ice frost point for a duration greater than 1.5 days than with either the region bounded by the NAT existence threshold $\left(T<T_{\mathrm{NAT}}\right)$ or the region of minimum temperatures close to $T_{\mathrm{STS}}$. We infer a large particle radius from the estimated sedimentation rate, which caused the vertical redistribution of $\mathrm{HNO}_{3}$ from the $32-46 \mathrm{hPa}$ pressure levels to $68 \mathrm{hPa}$ in less than four days. This denitrification event is observed in the central region of the Antarctic vortex close to the pole, before synoptic temperatures first fall below the ice frost point.

We have investigated an outbreak of large-scale NAT PSCs in the polar freezing belt in an episode of early Antarctic winter behavior similar to that originally observed by MIPAS in mid-June 2003 (Höpfner et al., 2006a; Eckermann et al., 2009). Small particle NAT PSCs with estimated effective radii of around $1 \mu \mathrm{m}$ and high NAT number densities $>0.2 \mathrm{~cm}^{-3}$ were observed forming at altitudes $18-26 \mathrm{~km}$ downstream of gravity-wave activity and ice formation over the Palmer Peninsula and Ellsworth Mountains starting from 28 May 2008 and extending until at least 3 June 2008. These NAT PSCS are clearly identified from the high backscatter and depolarization detected by the CALIOP lidar (Pitts et al., 2011) and represent a distinct sub-class of Type Ia PSCs previously described by Tsias et al. (1999) and Hu et al. (2002). The MIPAS data were used to show that the PSCs in the polar belt also show a characteristic $820 \mathrm{~cm}^{-1}$ NAT infrared spectral signature (Höpfner et al., 2006b). Forward trajectory calculations from the times and locations of the ice detections by MIPAS and CALIOP indicated origination of the NAT clouds via ice-seeding in the rapid cooling phases of gravity waves that were identified by the AIRS instrument to be active over the Palmer Peninsula starting from 28 May 2008. 
The combination of CALIOP and MLS observations over several years provides more extensive temporal and spatial data for aerosols, $\mathrm{HNO}_{3}, \mathrm{H}_{2} \mathrm{O}$, and $\mathrm{O}_{3}$, and other important species such as $\mathrm{HCl}$ and $\mathrm{ClO}$, than previously available. These near-simultaneous measurements provide a unique opportunity to capture the various stages of PSC development in relation to the interactions of the ambient temperature, temperature exposure history, and gas-phase species during the PSC life-cycle. This work presented case studies showing correlations of the $\mathrm{HNO}_{3}$ depletion with the spatial distribution of the temperature threshold exposure. We intend to undertake a statistical study of the formation of PSCs and the evolution of denitrification events in the vortex that should provide a stringent examination of the effects of temperature history on NAT formation.

Acknowledgements. We gratefully acknowledge all of the teams associated with the AIRS, CALIOP, MLS and MIPAS instruments, and the GEOS-5 meteorological analyses. AIRS and MLS data were obtained from the NASA Goddard Earth Sciences Data Information and Services Center. CALIOP data were obtained from the NASA Langley Research Center Atmospheric Science Data Center. EnviSat MIPAS Level-1 data were provided by the European Space Agency. We thank A. Dudhia for supplying MIPAS cloud top height data and maintaining an online resource of MIPAS related information (http://www.atm.ox.ac.uk/group/mipas/). IDL software for calculation of PSC thermodynamic properties provided by M. E. Hervig was obtained from the GATS Scientific Software website (http://gwest.gats-inc.com/software/software_page.html). Fortran software for $T$-matrix calculations provided by M. I. Mishchenko was obtained from the NASA GISS website (http://www.giss.nasa.gov/staff/mmishchenko/t_matrix.html). We are grateful to M. C. Pitts and L. R. Poole for their comments on an early version of the manuscript. Work at the Jet Propulsion Laboratory, California Institute of Technology, was carried out under a contract with the National Aeronautics and Space Administration.

Edited by: W. Lahoz

\section{References}

Adhikari, L., Wang, Z., and Liu, D.: Microphysical properties of Antarctic polar stratospheric clouds and their dependence on tropospheric cloud systems, J. Geophys. Res., 115, D00H18, doi:10.1029/2009JD012125, 2010.

Adriani, A., Massoli, P., Di Donfrancesco, G., Cairo, F., Moriconi, M. L., and Snels, M.: Climatology of polar stratospheric clouds based on lidar observations from 1993 to 2001 over McMurdo Station, Antarctica, J. Geophys. Res., 109, D24211, doi:10.1029/2004JD004800, 2004.

Aguzzi, A. and Rossi, M. J.: The kinetics of the uptake of $\mathrm{HNO}_{3}$ on ice, solid $\mathrm{H}_{2} \mathrm{SO}_{4}-\mathrm{H}_{2} \mathrm{O}$ and solid ternary solutions of $\mathrm{H}_{2} \mathrm{SO}_{4}$ $\mathrm{HNO}_{3}-\mathrm{H}_{2} \mathrm{O}$ in the temperature range $180-211 \mathrm{~K}$, Phys. Chem. Chem. Phys., 3, 3707-3716, doi:10.1039/b100546o, 2001.

Alexander, S. P., Klekociuk, A. R., Pitts, M. C., McDonald, A. J., and Arevalo-Torres, A.: The effect of orographic gravity waves on Antarctic polar stratospheric cloud occurrence and composition, J. Geophys. Res., 116, D06109, doi:10.1029/2010JD015184, 2011.

Arnold, F., Bürger, V., Gollinger, K., Roncossek, M., Schneider, J., and Spreng, S.: Observations of nitric acid perturbations in the winter Arctic stratosphere: evidence for PSC sedimentation, J. Atmos. Chem., 30, 49-59, 1998.

Aumann, H. H., Chahine, M. T., Gautier, C., Goldberg, M. D., Kalnay, E., McMillin, L. M., Revercomb, H., Rosenkranz, P. W., Smith, W. L., Staelin, D. H., Strow, L. L., and Susskind, J.: AIRS/AMSU/HSB on the Aqua Mission: design, science objectives, data products, and processing systems, IEEE T. Geosci. Remote Sens., 41, 253-264, 2003.

Biele, J., Tsias, A., Luo, B. P., Carslaw, K. S., Neuber, R., Beyerle, G., and Peter, T.: Nonequilibrium coexistence of solid and liquid particles in Arctic stratospheric clouds, J. Geophys. Res., 106, 22991-23007, 2001.

Biermann, U. M., Presper, T., Mößinger, J., Crutzen, P. J., and Peter, T.: The unsuitability of meteoritic and other nuclei for polar stratospheric cloud freezing, Geophys. Res. Lett., 23, 16931696, 1996.

Biermann, U. M., Crowley, J. N., Huthwelker, T., Moortgat, G. K., Crutzen, P. J., and Peter, T.: FTIR studies on lifetime prolongation of stratospheric ice particles due to NAT coating, Geophys. Res. Lett., 25, 3939-3942, 1998.

Brooks, S. D., Toon, O. B., Tolbert, M. A., Baumgardner, D., Gandrud, B., Browell, E. V., Flentje, H., and Wilson, J. C.: Polar stratospheric clouds during SOLVE/THESEO: Comparison of lidar observations with in situ measurements, J. Geophys. Res., 109, D02212, doi:10.1029/2003JD003463, 2004.

Browell, E. V., Butler, C. F., Ismail, S., Robinette, P. A., Carter, A. F., Higdon, N. S., Toon, O. B., Schoeberl, M. R., and Tuck, A. F.: Airborne lidar observations in the wintertime Arctic stratosphere: polar stratospheric clouds, Geophys. Res. Lett., 17, 385-388, 1990.

Cariolle, D., Muller, S., Cayla, F., and McCormick, M. P.: Mountain waves, polar stratospheric clouds, and the ozone depletion over Antarctica, J. Geophys. Res., 94, 11233-11240, 1989.

Carlsaw, K. S., Luo, B. P., Clegg, S. L., Peter, T., Brimblecombe, P., and Crutzen, P. J.: Stratospheric aerosol growth and $\mathrm{HNO}_{3}$ gas phase depletion from coupled $\mathrm{HNO}_{3}$ and water uptake by liquid particles, Geophys. Res. Lett., 21, 2479-2482, 1994.

Carlsaw, K. S., Luo, B. P., and Peter, T.: An analytic expression for the composition of aqueous $\mathrm{HNO}_{3}-\mathrm{H}_{2} \mathrm{SO}_{4}$ stratospheric aerosols including gas phase removal of $\mathrm{HNO}_{3}$, Geophys. Res. Lett., 22, 1877-1880, 1995.

Carslaw, K. S., Peter, T., and Clegg, S. L.: Modeling the composition of liquid stratospheric aerosols, Rev. Geophys., 35, 125-154, 1997.

Carslaw, K. S., Wirth, M., Tsias, A., Luo, B. P., Dörnbrack, A., Leutbecher, M., Volkert, H., Renger, W., Bacmeister, J. T., Reimer, E., and Peter, T.: Increased stratospheric ozone depletion due to mountain-induced atmospheric waves, Nature, 391, 675-678, 1998.

Carslaw, K. S., Kettleborough, J. A., Northway, M. J., Davies, S., Gao, R.-S., Fahey, D. W., Baumgardner, D. G., Chipperfield, M. P., and Kleinböhl, A.: A vortex-scale simulation of the growth and sedimentation of large nitric acid hydrate particles, J. Geophys. Res., 107, 8300, doi:10.1029/2001JD000467, 2002.

Daneva, D., Shibata, T., Iwasaka, Y., Nagatani, M., Shiraishi, K., 
Hayashi, M., Fujiwara, M., and Neuber, R.: The mixing state of polar stratospheric cloud particles in sandwich structure observed by lidar 1. Determination of the mixing state of PSC particles, J. Meteorol. Soc. Japan, 8, 747-757, 2003.

Danilin, M. Y., Ko, M. K. W., Froidevaux, L., Santee, M. L., Lyjak, L. V., Bevilacqua, R. M., Zawodny, J. M., Sasano, Y., Irie, H., Kondo, Y., Russell, J. M., Scott, C. J., and Read, W. G.: Trajectory hunting as an effective technique to validate multiplatform measurements: analysis of the MLS, HALOE, SAGE-II, ILAS, and POAM-II data in October-November 1996, J. Geophys. Res., 107, 4420, doi:10.1029/2001JD002012, 2002.

Dhaniyala, S., McKinney, K. A., and Wennberg, P. O.: Lee-wave clouds and denitrification of the polar stratosphere, Geophys. Res. Lett., 29, 36, doi:10.1029/2001GL013900, 2002.

Dibb, J. E., Scheuer, E., Avery, M., Plant, J., and Sachse, G.: In situ evidence for renitrification in the Arctic lower stratosphere during the polar Aura validation experiment (PAVE), Geophys. Res. Lett., 33, L12815, doi:10.1029/2006GL026243, 2006.

Drdla, K., Schoeberl, M. R., and Browell, E. V.: Microphysical modeling of the 1999-2000 Arctic winter: 1. Polar stratospheric clouds, denitrification, and dehydration, J. Geophys. Res., 107, 8312, doi:10.1029/2001JD000782, 2002.

Drdla, K., Gandrud, B. W., Baumgardner, D., Wilson, J. C., Bui, T. P., Hurst, D., Schauffler, S. M., Jost, H., Greenblatt, J. B., and Webster, C. R.: Evidence for the widespread presence of liquid-phase particles during the 1999-2000 Arctic winter, J. Geophys. Res., 108, 8318, doi:10.1029/2001JD001127, 2003.

Dye, J. E., Baumgardner, D., Gandrud, B. W., Kawa, S. R., Kelly, K. K., Loewenstein, M., Ferry, G. V., Chan, K. R., and Gary, B. L.: Particle size distributions in Arctic polar stratospheric clouds, growth and freezing of sulfuric acid droplets, and implications for cloud formation, J. Geophys. Res., 97, 80158034, 1992.

Dye, J. E., Baumgardner, D., Gandrud, B. W., Drdla, K., Barr, K., Fahey, D. W., Delnegro, L. A., Tabazadeh, A., Jonsson, H. H., Wilson, J. C., Loewenstein, M., Podolske, J. R., and Chan, K. R.: In-situ observations of an Antarctic polar stratospheric cloud: similarities with Arctic observations, Geophys. Res. Lett., 23, 1913-1916, 1996.

Eckermann, S. D., Wu, D. L., Doyle, J. D., Burris, J. F., McGee, T. J., Hostetler, C. A., Coy, L., Lawrence, B. N., Stephens, A., McCormack, J. P., and Hogan, T. F.: Imaging gravity waves in lower stratospheric AMSU-A radiances, Part 2: Validation case study, Atmos. Chem. Phys., 6, 3343-3362, doi:10.5194/acp-6-3343-2006, 2006.

Eckermann, S. D., Hoffmann, L., Hoepfner, M., Wu, D. L., and Alexander, M. J.: Antarctic NAT PSC belt of June 2003: Observational validation of the mountain wave seeding hypothesis, Geophys. Res. Lett., 36, L02807, doi:10.1029/2008GL036629, 2009.

Fahey, D. W., Kelly, K. K., Kawa, S. R., Tuck, A. F., Loewenstein, M., Chan, K. R., and Heidt, L. E.: Observations of denitrification and dehydration in the winter polar stratospheres, Nature, 344, 321-324, 1990.

Fahey, D. W., Gao, R. S., Carslaw, K. S., Kettleborough, J., Popp, P. J., Northway, M. J., Holecek, J. C., Ciciora, S. C., McLaughlin, R. J., Thompson, T. L., Winkler, R. H., Baumgardner, D. G., Gandrud, B., Wennberg, P. O., Dhaniyala, S., McKinney, K., Peter, T., Salawitch, R. J., Bui, T. P., Elkins, J. W., Web- ster, C. R., Atlas, E. L., Jost, H., Wilson, J. C., Herman, R. L., Kleinbohl, A., and von Konig, M.: The detection of large $\mathrm{HNO}_{3}-$ containing particles in the winter Arctic stratosphere, Science, 291, 1026-1031, 2001.

Fischer, H., Birk, M., Blom, C., Carli, B., Carlotti, M., von Clarmann, T., Delbouille, L., Dudhia, A., Ehhalt, D., Endemann, M., Flaud, J. M., Gessner, R., Kleinert, A., Koopman, R., Langen, J., López-Puertas, M., Mosner, P., Nett, H., Oelhaf, H., Perron, G., Remedios, J., Ridolfi, M., Stiller, G., and Zander, R.: MIPAS: an instrument for atmospheric and climate research, Atmos. Chem. Phys., 8, 2151-2188, doi:10.5194/acp-8-2151-2008, 2008.

Fromm, M., Alfred, J., and Pitts, M.: A unified, long-term, high-latitude stratospheric aerosol and cloud database using SAM II, SAGE II, and POAM II/III data: algorithm description, database definition, and climatology, J. Geophys. Res., 108, 4366, doi:10.1029/2002JD002772, 2003.

Fueglistaler, S., Luo, B. P., Voigt, C., Carslaw, K. S., and Peter, Th.: NAT-rock formation by mother clouds: a microphysical model study, Atmos. Chem. Phys., 2, 93-98, doi:10.5194/acp-2-932002, 2002a.

Fueglistaler, S., Luo, B. P., Buss, S., Wernli, H., Voigt, C., Müller, M., Neuber, R., Hostetler, C. A., Poole, L. R., Flentje, H., Fahey, D. W., Northway, M. J., and Peter, Th.: Large NAT particle formation by mother clouds: analysis of SOLVE/THESEO-2000 observations, Geophys. Res. Lett., 29, 1610, doi:10.1029/2001GL014548, 2002 b.

Hanson, D. and Mauersberger, K.: Laboratory studies of the nitric acid trihydrate: implications for the south polar stratosphere, Geophys. Res. Lett., 15, 855-858, 1988.

Hatch, C. D., Gough, R. V., Toon, O. B., and Tolbert, M. A.: Heterogeneous nucleation of nitric acid trihydrate on clay minerals: relevance to type Ia polar stratopsheric clouds, J. Phys. Chem. B., 112, 612-620, doi:10.1021/jp075828n, 2008.

Hervig, M. E.: Stratospheric clouds over England, Geophys. Res. Lett., 26, 1137-1140, 1999.

Hoffmann, L. and Alexander, M. J.: Retrieval of stratospheric temperatures from Atmospheric Infrared Sounder radiance measurements for gravity wave studies, J. Geophys. Res., 114, D07105, doi:10.1029/2008JD011241, 2009.

Hofmann, D. J. and Deshler, T.: Stratospheric cloud observations during formation of the Antarctic ozone hole in 1989, J. Geophys. Res., 96, 2897-2912, 1991.

Höpfner, M., Larsen, N., Spang, R., Luo, B. P., Ma, J., Svendsen, S. H., Eckermann, S. D., Knudsen, B., Massoli, P., Cairo, F., Stiller, G., v. Clarmann, T., and Fischer, H.: MIPAS detects Antarctic stratospheric belt of NAT PSCs caused by mountain waves, Atmos. Chem. Phys., 6, 1221-1230, doi:10.5194/acp-61221-2006, 2006a.

Höpfner, M., Luo, B. P., Massoli, P., Cairo, F., Spang, R., Snels, M., Di Donfrancesco, G., Stiller, G., von Clarmann, T., Fischer, H., and Biermann, U.: Spectroscopic evidence for NAT, STS, and ice in MIPAS infrared limb emission measurements of polar stratospheric clouds, Atmos. Chem. Phys., 6, 1201-1219, doi:10.5194/acp-6-1201-2006, 2006b.

Höpfner, M., Pitts, M. C., and Poole, L. R.: Comparison between CALIPSO and MIPAS observations of polar stratospheric clouds, J. Geophys. Res., 114, D00H05, doi:10.1029/2009JD012114, 2009.

Hostetler, C. A., Liu, Z., Reagan, J., Vaughan, M., Winker, D., 
Osborn, M., Hunt, W. H., Powell, K. A., and Trepte, C.: CALIOP Algorithm Theoretical Basis Document: Calibration and Level 1 Products, Tech. rep., NASA Langley Research Center Document PC-SCI-201, Hampton, V. A., available at: http://www-calipso.larc.nasa.gov/resources/pdfs/ PC-SCI-201v1.0.pdf, last access: October 2011, 2006.

Hu, R.-M., Carslaw, K. S., Hostetler, C., Poole, L. R., Luo, B., Peter, T., Füeglistaler, S., McGee, T. J., and Burris, J. F.: Microphysical properties of wave polar stratospheric clouds retrieved from lidar measurements during SOLVE/THESEO 2000, J. Geophys. Res., 107, 8294, doi:10.1029/2001JD001125, 2002.

Hübler, G., Fahey, D. W., Kelly, K. K., Montzka, D. D., Carroll, M. A., Tuck, A. F., Heidt, L. E., Pollock, W. H., Gregory, G. L., and Vedder, J. F.: Redistribution of reactive odd nitrogen in the lower Arctic stratosphere, Geophys. Res. Lett., 17, 453-456, 1990.

Hunt, W. H., Winker, D. M., Vaughan, M. A., Powell, K. A., Lucker, P. L., and Weimer, C.: CALIPSO lidar description and performance assessment, J. Atmos. Oceanic Technol., 26, 12141228, doi:10.1175/2009JTECHA1223.1, 2009.

Hurley, J., Dudhia, A., and Grainger, R. G.: Retrieval of macrophysical cloud parameters from MIPAS: algorithm description, Atmos. Meas. Tech., 4, 683-704, doi:10.5194/amt-4-683-2011, 2011.

Irie, H., Koike, M., Kondo, Y., Bodeker, G. E., Danilin, M. Y., and Sasano, Y.: Redistribution of nitric acid in the Arctic lower stratosphere during the winter of 1996-1997, J. Geophys. Res., 106, 23139-23150, 2001.

Jarnot, R. F., Perun, V. S., and Schwartz, M. J.: Radiometric and spectral performance and calibration of the $\mathrm{GHz}$ bands of EOS MLS, IEEE T. Geosci. Remote, 44, 1131-1143, 2006.

Jensen, E. J., Toon, O. B., Tabazadeh, A., and Drdla, K.: Impact of polar stratospheric cloud particle composition, number density, and lifetime on denitrification, J. Geophys. Res., 107, 8284, doi:10.1029/2001JD000440, 2002.

Jin, J. J., Semeniuk, K., Manney, G. L., Jonsson, A. I., Beagley, S. R., McConnell, J. C., Rinsland, C. P., Boone, C. D., Walker, K. A., and Bernath, P. F.: Denitrification in the Arctic winter 2004/2005: observations from ACE-FTS, Geophys. Res. Lett., 33, L19814, doi:10.1029/2006GL027687, 2006.

Kinne, S., Toon, O. B., Toon, G. C., Farmer, C. B., Browell, E. V., and McCormick, M. P.: Measurements of size and composition of particles in polar stratospheric clouds from infrared solar absorption spectra, J. Geophys. Res., 94, 16481-16491, 1989.

Koike, M., Kondo, Y., Takegawa, N., Lefevre, F., Ikeda, H., Irie, H., Hunton, H. D. E., Viggiano, A. A., Miller, T. M., Ballenthin, J. O., Sachse, G. W., Anderson, B. E., Avery, M., and Masui, Y.: Redistribution of reactive nitrogen in the Arctic lower stratosphere in the 1999/2000 winter, J. Geophys. Res., 107, 8275, doi:10.1029/2001JD001089, 2002.

Kondo, Y., Irie, H., Koike, M., and Bodeker, G. E.: Denitrification and nitrification in the Arctic stratosphere during the winter of 1996-1997, Geophys. Res. Lett., 27, 337-340, 2000.

Koop, T., Carslaw, K. S., and Peter, T.: Thermodynamic stability and phase transitions of PSC particles, Geophys. Res. Lett., 24, 2199-2202, 1997a.

Koop, T., Luo, B., Biermann, U. M., Crutzen, P. J., and Peter, T.: Freezing of $\mathrm{HNO}_{3} / \mathrm{H}_{2} \mathrm{SO}_{4} / \mathrm{H}_{2} \mathrm{O}$ solutions at stratospheric temperatures: nucleation statistics and experiments, J. Phys. Chem.
A, 101, 1117-1133, 1997b.

Koop, T., Ng, H. P., Molina, L. T., and Molina, M. J.: A new optical technique to study aerosol phase transitions: the nucleation of ice from $\mathrm{H}_{2} \mathrm{SO}_{4}$ aerosols, J. Phys. Chem. A, 102, 8924-8931, 1998.

Lambert, A., Read, W. G., Livesey, N. J., Santee, M. L., Manney, G. L., Froidevaux, L., Wu, D. L., Schwartz, M. J., Pumphrey, H. C., Jimenez, C., Nedoluha, G. E., Cofield, R. E., Cuddy, D. T., Daffer, W. H., Drouin, B. J., Fuller, R. A., Jarnot, R. F., Knosp, B. W., Pickett, H. M., Perun, V. S., Snyder, W. V., Stek, P. C., Thurstans, R. P., Wagner, P. A., Waters, J. W., Jucks, K. W., Toon, G. C., Stachnik, R. A., Bernath, P. F., Boone, C. D., Walker, K. A., Urban, J., Murtagh, D., Elkins, J. W., and Atlas, E.: Validation of the Aura Microwave Limb Sounder middle atmosphere water vapor and nitrous oxide measurements, J. Geophys. Res., 112, D24S36, doi:10.1029/2007JD008, 2007.

Larsen, N., Knudsen, B. M., Rosen, J. M., Kjome, N. T., Neuber, R., and Kyrö, E.: Temperature histories in liquid and solid polar stratospheric cloud formation, J. Geophys. Res., 102, 2350523517, 1997.

Larsen, N., Knudsen, B. M., Svendsen, S. H., Deshler, T., Rosen, J. M., Kivi, R., Weisser, C., Schreiner, J., Mauerberger, K., Cairo, F., Ovarlez, J., Oelhaf, H., and Spang, R.: Formation of solid particles in synoptic-scale Arctic PSCs in early winter 2002/2003, Atmos. Chem. Phys., 4, 2001-2013, doi:10.5194/acp-4-2001-2004, 2004.

Liu, L. and Mishchenko, M. I.: Constraints on PSC particle microphysics derived from lidar observations, J. Quant. Spectrosc. Ra., 70, 817-831, doi:10.1016/S0022-4073(01)00048-6, 2001.

Livesey, N. J., Snyder, W. V., Read, W. G., and Wagner, P. A.: Retrieval algorithms for the EOS Microwave Limb Sounder (MLS), IEEE T. Geosci. Remote, 44, 1144-1155, 2006.

Livesey, N. J., Read, W. G., Lambert, A., Cofield, R. E., Cuddy, D. T., Froidevaux, L., Fuller, R. A., Jarnot, R. F., Jiang, J. H., Jiang, Y. B., Knosp, B. W., Kovalenko, L. J., Pickett, H. M., Pumphrey, H. C., Santee, M. L., Schwartz, M. J., Stek, P. C., Wagner, P. A., Waters, J. W.,, and Wu, D. L.: Version 2.2 Level 2 data quality and description document, Tech. Rep. JPL D-32381, Jet Propulsion Laboratory, available at: http: //mls.jpl.nasa.gov., last access: October 2011, 2008.

Livesey, N. J., Read, W. G., Froidevaux, L., Lambert, A., Manney, G. L., Pumphrey, H. C., Santee, M. L., Schwartz, M. J., Wang, S., Cofield, R. E., Cuddy, D. T., Fuller, R. A., Jarnot, R. F., Jiang, J. H., Knosp, B. W., Stek, P. C., Wagner, P. A., and Wu, D. L.: Version 3.3 Level 3 data quality and description document, Tech. Rep. JPL D-33509, Jet Propulsion Laboratory, available at: http://mls.jpl.nasa.gov., last access: October 2011, 2011.

Lowe, D. and MacKenzie, A. R.: Polar stratospheric cloud microphysics and chemistry, J. Atmos. Solar-Terr. Phys., 70, 13-40, doi:10.1016/j.jastp.2007.09.011, 2008.

Lowe, D., MacKenzie, A. R., Schlager, H., Voigt, C., Dörnbrack, A., Mahoney, M. J., and Cairo, F.: Liquid particle composition and heterogeneous reactions in a mountain wave Polar Stratospheric Cloud, Atmos. Chem. Phys., 6, 3611-3623, doi:10.5194/acp-6-3611-2006, 2006.

Massie, S., Dye, J., Baumgardner, D., Randel, W., Wu, F., Tie, X., Pan, L., Figarol, F., Brasseur, G., Santee, M., Read, W., Grainger, R., Lambert, A., Mergenthaler, J., and Tabazadeh, A.: Simultaneous observations of polar stratospheric clouds and 
$\mathrm{HNO}_{3}$ over Scandinavia in January, 1992, Geophys. Res. Lett., 24, 595-598, 1997.

McDonald, A. J., George, S. E., and Woollands, R. M.: Can gravity waves significantly impact PSC occurrence in the Antarctic?, Atmos. Chem. Phys., 9, 8825-8840, doi:10.5194/acp-9-8825-2009, 2009.

McKinney, K., Wennberg, P., Dhaniyala, S., Fahey, D., Northway, M., Kunzi, K., Kleinbohl, A., Sinnhuber, M., Kullmann, H., Bremer, H., Mahoney, M., and Bui, T.: Trajectory studies of large $\mathrm{HNO}_{3}$-containing PSC particles in the Arctic: evidence for the role of NAT, Geophys. Res. Lett., 31, L05110, doi:10.1029/2003GL018430, 2004.

Meilinger, S. K., Koop, T., Luo, B. P., Huthwelker, T., Carslaw, K. S., Krieger, U., Crutzen, P. J., and Peter, T.: Sizedependent stratospheric droplet composition in lee wave temperature fluctuations and their potential role in PSC freezing, Geophys. Res. Lett., 22, 3031-3034, 1995.

Mishchenko, M. I. and Travis, L. D.: Capabilities and limitations of a current FORTRAN implementation of the $T$-matrix method for randomly oriented, rotationally symmetric scatterers, J. Quant. Spectrosc. Ra., 60, 309-324, doi:10.1016/S00224073(98)00008-9, 1998.

Murphy, D. M. and Koop, T.: Review of the vapour pressures of ice and supercooled water for atmospheric applications, Q. J. Roy. Meteor. Soc., 131, 1539-1565, 2005.

Noel, V., Hertzog, A., Chepfer, H., and Winker, D. M.: Polar stratospheric clouds over Antarctica from the CALIPSO spaceborne lidar, J. Geophys. Res., 113, D02205, doi:10.1029/2007JD008616, 2008.

Noel, V., Hertzog, A., and Chepfer, H.: CALIPSO observations of wave-induced PSCs with near-unity optical depth over Antarctica in 2006-2007, J. Geophys. Res., 114, D05202, doi:10.1029/2008JD010604, 2009.

Northway, M. J., Gao, R. S., Popp, P. J., Holecek, J. C., Fahey, D. W., Carslaw, K. S., Tolbert, M. A., Lait, L. R., Dhaniyala, S., Flagan, R. C., Wennberg, P. O., Mahoney, M. J., Herman, R. L., Toon, G. C., and Bui, T. P.: An analysis of large $\mathrm{HNO}_{3}$-containing particles sampled in the Arctic stratosphere during the winter of 1999/2000, J. Geophys. Res., 107, 8298, doi:10.1029/2001JD001079, 2002.

Pagan, K. L., Tabazadeh, A., Drdla, K., Hervig, M. E., Eckermann, S. D., Browell, E. V., Legg, M. J., and Foschi, P. G.: Observational evidence against mountain-wave generation of ice nuclei as a prerequisite for the formation of three solid nitric acid polar stratospheric clouds observed in the Arctic in early December 1999, J. Geophys. Res., 109, D04312, doi:10.1029/2003JD003846, 2004.

Peter, T.: Microphysics and heterogeneous chemistry of polar stratospheric clouds, Annu. Rev. Phys. Chem., 48, 785-822, 1997.

Peter, T., Müller, R., Crutzen, P. J., and Deshler, T.: The lifetime of leewave-induced ice particles in the Arctic stratosphere: II, Stabilization due to NAT-coating, Geophys. Res. Lett., 21, 13311334, 1994.

Pitts, M. C., Thomason, L. W., Poole, L. R., and Winker, D. M.: Characterization of Polar Stratospheric Clouds with spaceborne lidar: CALIPSO and the 2006 Antarctic season, Atmos. Chem. Phys., 7, 5207-5228, doi:10.5194/acp-7-5207-2007, 2007.

Pitts, M. C., Poole, L. R., and Thomason, L. W.: CALIPSO polar stratospheric cloud observations: second-generation detection algorithm and composition discrimination, Atmos. Chem. Phys., 9, 7577-7589, doi:10.5194/acp-9-7577-2009, 2009.

Pitts, M. C., Poole, L. R., Dörnbrack, A., and Thomason, L. W.: The 2009-2010 Arctic polar stratospheric cloud season: a CALIPSO perspective, Atmos. Chem. Phys., 11, 2161-2177, doi:10.5194/acp-11-2161-2011, 2011.

Read, W. G., Lambert, A., Bacmeister, J., Cofield, R. E., Christensen, L. E., Cuddy, D. T., Daffer, W. H., Drouin, B. J., Fetzer, E., Froidevaux, L., Fuller, R., Herman, R., Jarnot, R. F., Jiang, J. H., Jiang, Y. B., Kelly, K., Knosp, B. W., Kovalenko, L. J., Livesey, N. J., Liu, H. C., Manney, G. L., Pickett, H. M., Pumphrey, H. C., Rosenlof, K. H., Sabounchi, X., Santee, M. L., Schwartz, M. J., Snyder, W. V., Stek, P. C., Su, H., Takacs, L. L., Thurstans, R. P., Vomel, H., Wagner, P. A., Waters, J. W., Webster, C. R., Weinstock, E. M., and Wu, D. L.: Aura Microwave Limb Sounder upper tropospheric and lower stratospheric $\mathrm{H}_{2} \mathrm{O}$ and relative humidity with respect to ice validation, J. Geophys. Res., 112, D24S35, doi:10.1029/2007JD008752, 2007.

Reichardt, J., Reichardt, S., Yang, P., and McGee, T. J.: Retrieval of polar stratospheric cloud microphysical properties from lidar measurements: dependence on particle shape assumptions, J Geophys. Res., 107, 8282, doi:10.1029/2001JD001021, 2002.

Reichardt, J., Dörnbrack, A., Reichardt, S., Yang, P., and McGee, T. J.: Mountain wave PSC dynamics and microphysics from ground-based lidar measurements and meteorological modeling, Atmos. Chem. Phys., 4, 1149-1165, doi:10.5194/acp-4-11492004, 2004.

Ricaud, P. D., Carr, E. S., Harwood, R. S., Lahoz, W. A., Froidevaux, L., Read, W. G., Waters, J. W., Mergenthaler, J. L., Kumer, J. B., Roche, A. E., and Peckham, G. E.: Polar stratospheric clouds as deduced from MLS and CLAES measurements, Geophys. Res. Lett., 22, 2033-2036, 1995.

Rienecker, M. M., Suarez, M. J., Todling, R., Bacmeister, K., Takacs, L., Liu, H.-C., Gu, W., Sienkiewicz, M., Koster, R. D., Gelaro, R., Stajner, I., and Nielsen, J. E.: The GEOS-5 Data Assimilation System - Documentation of Versions 5.0.1, 5.1.0, and 5.2.0, Tech. Rep., NASA Goddard Space Flight Center, Greenbelt, MD., NASA/TM-2008-10406, Vol. 27, 2008.

Rodgers, C. D.: Inverse Methods for Atmospheric Sounding: Theory and Practice, World Sci., Singapore, 2000.

Santee, M. L., Manney, G. L., Livesey, N. J., and Waters, J. W.: UARS Microwave Limb Sounder observations of denitrification and ozone loss in the 2000 Arctic late winter, Geophys. Res. Lett., 27, 3213-3216, 2000.

Santee, M. L., Lambert, A., Read, W. G., Livesey, N. J., Cofield, R. E., Cuddy, D. T., Daffer, W. H., Drouin, B. J., Froidevaux, L., Fuller, R. A., Jarnot, R. F., Knosp, B. W., Manney, G. L., Perun, V. S., Snyder, W. V., Stek, P. C., Thurstans, R. P., Wagner, P. A., Waters, J. W., Muscari, G., de Zafra, R. L., Dibb, J. E., Fahey, D. W., Popp, P. J., Marcy, T. P., Jucks, K. W., Toon, G. C., Stachnik, R. A., Bernath, P. F., Boone, C. D., Walker, K. A., Urban, J., and Murtagh, D.: Validation of the Aura Microwave Limb Sounder $\mathrm{HNO}_{3}$ measurements, J. Geophys. Res., 112, D24S40, doi:10.1029/2007JD008, 2007.

Schoeberl, M. R., Douglass, A. R., Hilsenrath, E., Bhartia, P. K., Beer, R., Waters, J. W., Gunson, M. R., Froidevaux, L., 
Gille, J. C., Barnett, J. J., Levelt, P., and DeCola, P.: Overview of the EOS Aura mission, IEEE T. Geosci. Remote, 44, 1066-1074, 2006.

Schwartz, M. J., Manney, G. L., Lambert, A., Read, W. G., Livesey, N. J., Waters, J. W., Wu, D. L., Froidevaux, L., Ao, C. O., Cofield, R. E., Daffer, W. H., Drouin, B. J., Fetzer, E. J., Fuller, R. A., Jarnot, R. F., Jiang, J. H., Jiang, Y. B., Knosp, B. W., Li, J.-L. F., Santee, M. L., Snyder, W. V., Stek, P. C., Thurstans, R. P., Wagner, P. A., Pawson, S., Bernath, P. F., Walker, K. A., Boone, C. D., III, J. M. R., Mlynczak, M. G., Li, J.-L. F., Krüger, K., and Tompkins, A. M.: Validation of the Aura Microwave Limb Sounder temperature and geopotential height measurements, J. Geophys. Res., 113, D15S11, doi:10.1029/2007JD008783, 2008.

Solomon, S.: Stratospheric ozone depletion: a review of concepts and history, Rev. Geophys., 37, 275-316, 1999.

Spang, R. and Remedios, J. J.: Observations of a distinctive infrared spectral feature in the atmospheric spectra of polar stratospheric clouds measured by the CRISTA instrument, Geophys. Res. Lett., 30, 1875, doi:10.1029/2003GL017, 2003.

Spang, R., Remedios, J. J., and Barkley, M. P.: Colour indices for the detection and differentiation of cloud types in infra-red limb emision spectra, Adv. Space Res., 33, 1041-1047, 2004.

Steele, H. M., Lumpe, J. D., Bevilacqua, R. M., Hoppel, K. W., and Turco, R. P.: Role of temperature history in polar stratospheric cloud sightings, J. Geophys. Res., 107, 4618, doi:10.1029/2001JD001261, 2002.

Stefanutti, L., Morandi, M., Del Guasta, M., Godin, S., and David, C.: Unusual PSCs observed by LIDAR in Antarctica, Geophys. Res. Lett., 22, 2377-2380, 1995.

Tabazadeh, A., Santee, M. L., Danilin, M. Y., Pumphrey, H. C., Newman, P. A., Hamill, P. J., and Mergenthaler, J. L.: Quantifying denitrification and its effect on ozone recovery, Science, 288, 1407-1411, 2000.

Tabazadeh, A., Jensen, E. J., Toon, O. B., Drdla, K., and Schoeberl, M. R.: Role of the stratospheric polar freezing belt in denitrification, Science, 291, 2591-2594, 2001.

Taesler, I., Delaplane, R. G., and Olovsson, I.: Hydrogen bond studies. XCIV. Diaquaoxonium ion in nitric acid trihydrate, Acta Crystallogr., Sect. B, 31, 1489-1495, doi:10.1107/S056774087500550X, 1975.

Taylor, F., Lambert, A., Grainger, R., Rodgers, C., and Remedios, J.: Properties of Northern Hemisphere polar stratospheric clouds and volcanic aerosol in 1991/92 from UARS/ISAMS satellite measurements, J. Atmos. Sci., 51, 3019-3026, 1994.

Teitelbaum, H., Moustaoui, M., and Fromm, M.: Exploring polar stratospheric cloud and ozone minihole formation: the primary importance of synoptic-scale flow perturbations, J. Geophys. Res., 106, 28173-28188, 2001.

Thomason, L. W., Burton, S. P., Luo, B.-P., and Peter, T.: SAGE II measurements of stratospheric aerosol properties at non-volcanic levels, Atmos. Chem. Phys., 8, 983-995, doi:10.5194/acp-8-9832008, 2008.

Toon, O. B., Browell, E. V., Kinne, S., and Jordan, J.: An analysis of lidar observations of polar stratospheric clouds, Geophys. Res. Lett., 17, 393-396, 1990.

Toon, O. B., Tabazadeh, A., Browell, E. V., and Jordan, J.: Analysis of lidar observations of Arctic polar stratospheric clouds during January 1989, J. Geophys. Res., 105, 20589-20615, 2000.
Tsias, A., Wirth, M., Carslaw, K., Biele, J., Mehrtens, H., Reichardt, J., Wedekind, C., Weiss, V., Renger, W., Neuber, R., von Zahn, U., Stein, B., Santacesaria, V., Stefanutti, L., Fierli, F., Bacmeister, J., and Peter, T.: Aircraft lidar observations of an enhanced type Ia polar stratospheric cloud during APEPOLECAT, J. Geophys. Res., 104, 23961-23969, 1999.

Vernier, J. P., Pommereau, J. P., Garnier, A., Pelon, J., Larsen, N., Nielsen, J., Christiansen, T., Cairo, F., Thomason, L. W., Leblanc, T., and McDermid, I. S.: Tropical stratospheric aerosol layer from CALIPSO lidar observations, J. Geophys. Res., 114, D00H10, doi:10.1029/2009JD011, 2009.

Voigt, C., Schreiner, J., Kohlmann, A., Zink, P., Mauersberger, K., Larsen, N., Deshler, T., Kroger, C., Rosen, J., Adriani, A., Cairo, F., Di Donfrancesco, G., Viterbini, M., Ovarlez, J., Ovarlez, H., David, C., and Dornbrack, A.: Nitric acid trihydrate (NAT) in polar stratospheric clouds, Science, 290, 1756-1758, 2000a.

Voigt, C., Tsias, A., Dörnbrack, A., Meilinger, S., Luo, B., Schreiner, J., Larsen, N., Mauersberger, K., and Peter, T.: Nonequilibrium compositions of liquid polar stratospheric clouds in gravity waves, Geophys. Res. Lett., 27, 3873-3876, 2000 b.

Voigt, C., Schlager, H., Luo, B. P., Dörnbrack, A., Roiger, A., Stock, P., Curtius, J., Vössing, H., Borrmann, S., Davies, S., Konopka, P., Schiller, C., Shur, G., and Peter, T.: Nitric Acid Trihydrate (NAT) formation at low NAT supersaturation in Polar Stratospheric Clouds (PSCs), Atmos. Chem. Phys., 5, 13711380, doi:10.5194/acp-5-1371-2005, 2005.

von König, M., Bremer, H., Kleinbohl, A., Kullmann, H., Kunzi, K. F., Goede, A. P. H., Browell, E. V., Grant, W. B., Burris, J. F., McGee, T. J., and Twigg, L.: Using gas-phase nitric acid as an indicator of PSC composition, J. Geophys. Res., 107, 8265, doi:10.1029/2001JD001041, 2002.

Wagner, R., Möhler, O., Saathoff, H., Stetzer, O., and Schurath, U.: Infrared Spectrum of Nitric Acid Dihydrate: Influence of Particle Shape, J. Phys. Chem. A, 109, 2572-2581, doi:10.1021/jp044997u, 2005.

Wang, D. Y., Höpfner, M., Mengistu Tsidu, G., Stiller, G. P., von Clarmann, T., Fischer, H., Blumenstock, T., Glatthor, N., Grabowski, U., Hase, F., Kellmann, S., Linden, A., Milz, M., Oelhaf, H., Schneider, M., Steck, T., Wetzel, G., LópezPuertas, M., Funke, B., Koukouli, M. E., Nakajima, H., Sugita, T., Irie, H., Urban, J., Murtagh, D., Santee, M. L., Toon, G., Gunson, M. R., Irion, F. W., Boone, C. D., Walker, K., and Bernath, P. F.: Validation of nitric acid retrieved by the IMKIAA processor from MIPAS/ENVISAT measurements, Atmos. Chem. Phys., 7, 721-738, doi:10.5194/acp-7-721-2007, 2007.

Wang, Z., Stephens, G., Deshler, T., Trepte, C., Parish, T., Vane, D., Winker, D., Liu, D., and Adhikari, L.: Association of Antarctic polar stratospheric cloud formation on tropospheric cloud systems, Geophys. Res. Lett., 35, L13806, doi:10.1029/2008GL034209, 2008.

Waters, J. W., Froidevaux, L., Harwood, R. S., Jarnot, R. F., Pickett, H. M., Read, W. G., Siegel, P. H., Cofield, R. E., Filipiak, M. J., Flower, D. A., Holden, J. R., Lau, G. K. K., Livesey, N. J., Manney, G. L., Pumphrey, H. C., Santee, M. L., Wu, D. L., Cuddy, D. T., Lay, R. R., Loo, M. S., Perun, V. S., Schwartz, M. J., Stek, P. C., Thurstans, R. P., Boyles, M. A., Chandra, K. M., Chavez, M. C., Chen, G. S., Chudasama, B. V., Dodge, R., Fuller, R. A., Girard, M. A., Jiang, J. H., Jiang, Y. B., 
Knosp, B. W., LaBelle, R. C., Lam, J. C., Lee, K. A., Miller, D., Oswald, J. E., Patel, N. C., Pukala, D. M., Quintero, O., Scaff, D., Van Snyder, W., Tope, M. C., Wagner, P. A., and Walch, M. J.: The Earth Observing System Microwave Limb Sounder (EOS MLS) on the Aura satellite, IEEE Trans. Geosci. Remote Sens., 44, 1075-1092, 2006.

Winker, D. M., Vaughan, M. A., Omar, A., Hu, Y., Powell, K. A., Liu, Z., Hunt, W. H., and Young, S. A.: Overview of the CALIPSO Mission and CALIOP data processing algorithms, J. Atmos. Oceanic Technol., 26, 2310-2323, doi:10.1175/2009JTECHA1281.1, 2009.

Wofsy, S. C., Salawitch, R. J., Yatteau, J. H., McElroy, M. B., Gandrud, B. W., Dye, J. E., and Baumgardner, D.: Condensation of $\mathrm{HNO}_{3}$ on falling ice particles: Mechanism for denitrification of the polar stratosphere, Geophys. Res. Lett., 17, 449-452, 1990.
Worsnop, D. R., Fox, L. E., Zahniser, M. S., and Wofsy, S. C.: Vapor pressures of solid hydrates of nitric acid: Implications for polar stratospheric clouds, Science, 259, 71-74, 1993.

Wu, D. L., Chae, J. H., Lambert, A., and Zhang, F. F.: Characteristics of CALIOP attenuated backscatter noise: implication for cloud/aerosol detection, Atmos. Chem. Phys., 11, 2641-2654, doi:10.5194/acp-11-2641-2011, 2011.

Yu, F.: Formation of large NAT particles and denitrification in polar stratosphere: possible role of cosmic rays and effect of solar activity, Atmos. Chem. Phys., 4, 2273-2283, doi:10.5194/acp-42273-2004, 2004.

Zondlo, M., Hudson, P., Prenni, A., and Tolbert, M.: Chemistry and microphysics of polar stratospheric clouds and cirrus clouds, Ann. Rev. Phys. Chem., 51, 473-499, 2000. 